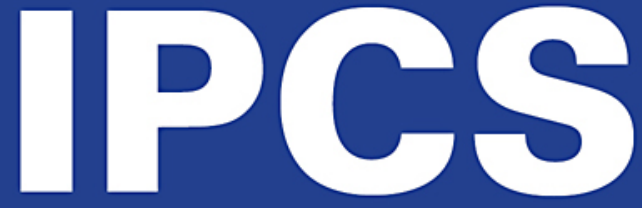

INTERNATIONAL PROGRAMME ON CHEMICAL SAFETY
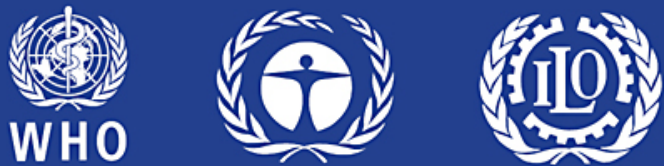

IPCS Harmonization Project

\title{
Characterization and Application of Physiologically Based Pharmacokinetic Models in Risk Assessment
}

\section{IOMC}

INTER-ORGANIZATION PROGRAMME FOR THE SOUND MANAGEMENT OF CHEMICALS

A cooperative agreement among FAO, ILO, UNEP, UNIDO, UNITAR, WHO, World Bank and OECD

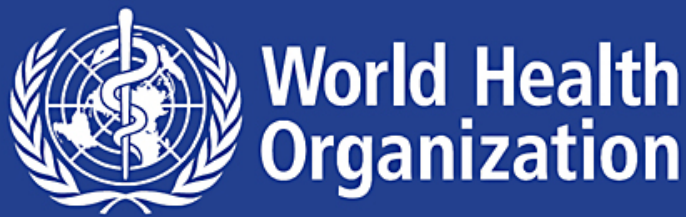


This report contains the collective views of an international group of experts and does not necessarily represent the decisions or the stated policy of the World Health Organization, the International Labour Organization or the United Nations Environment Programme.

Harmonization Project Document No. 9

\section{CHARACTERIZATION AND APPLICATION OF PHYSIOLOGICALLY BASED PHARMACOKINETIC MODELS IN RISK ASSESSMENT}

This project was conducted within the IPCS project on the Harmonization of Approaches to the Assessment of Risk from Exposure to Chemicals.

Published under the joint sponsorship of the World Health Organization, the International Labour Organization and the United Nations Environment Programme, and produced within the framework of the Inter-Organization Programme for the Sound Management of Chemicals. 
The International Programme on Chemical Safety (IPCS), established in 1980, is a joint venture of the United Nations Environment Programme (UNEP), the International Labour Organization (ILO) and the World Health Organization (WHO). The overall objectives of the IPCS are to establish the scientific basis for assessment of the risk to human health and the environment from exposure to chemicals, through international peer review processes, as a prerequisite for the promotion of chemical safety, and to provide technical assistance in strengthening national capacities for the sound management of chemicals.

The Inter-Organization Programme for the Sound Management of Chemicals (IOMC) was established in 1995 by UNEP, ILO, the Food and Agriculture Organization of the United Nations, WHO, the United Nations Industrial Development Organization, the United Nations Institute for Training and Research and the Organisation for Economic Co-operation and Development (Participating Organizations), following recommendations made by the 1992 UN Conference on Environment and Development to strengthen cooperation and increase coordination in the field of chemical safety. The purpose of the IOMC is to promote coordination of the policies and activities pursued by the Participating Organizations, jointly or separately, to achieve the sound management of chemicals in relation to human health and the environment.

WHO Library Cataloguing-in-Publication Data

Characterization and application of physiologically based pharmacokinetic models in risk assessment.

(IPCS harmonization project document ; no. 9)

1.Risk assessment. 2.Pharmacokinetics. 3.Chemicals - pharmacokinetics. 4.Chemicals - toxicity. 5.Drug toxicity. I.International Programme on Chemical Safety. II.Inter-Organization Programme for the Sound Management of Chemicals. III.Series.

ISBN $9789241500906 \quad$ (NLM classification: QV 38)

\section{(C) World Health Organization 2010}

All rights reserved. Publications of the World Health Organization can be obtained from WHO Press, World Health Organization, 20 Avenue Appia, 1211 Geneva 27, Switzerland (tel.: +41 22791 3264; fax: +41 22791 4857; e-mail: bookorders@who.int). Requests for permission to reproduce or translate WHO publications - whether for sale or for non-commercial distribution-should be addressed to WHO Press at the above address (fax: +41 22791 4806; e-mail: permissions@who.int).

The designations employed and the presentation of the material in this publication do not imply the expression of any opinion whatsoever on the part of the World Health Organization concerning the legal status of any country, territory, city or area or of its authorities, or concerning the delimitation of its frontiers or boundaries. Dotted lines on maps represent approximate border lines for which there may not yet be full agreement.

The mention of specific companies or of certain manufacturers' products does not imply that they are endorsed or recommended by the World Health Organization in preference to others of a similar nature that are not mentioned. Errors and omissions excepted, the names of proprietary products are distinguished by initial capital letters.

All reasonable precautions have been taken by the World Health Organization to verify the information contained in this publication. However, the published material is being distributed without warranty of any kind, either express or implied. The responsibility for the interpretation and use of the material lies with the reader. In no event shall the World Health Organization be liable for damages arising from its use.

Technically and linguistically edited by Marla Sheffer, Ottawa, Canada 


\section{TABLE OF CONTENTS}

FOREWORD 1

LIST OF ACRONYMS AND ABBREVIATIONS 2

PREFACE 3

1. INTRODUCTION 8

1.1 Context 8

$\begin{array}{lll}1.2 & \text { Objectives } & 9\end{array}$

1.3 Organization 9

2. TISSUE DOSIMETRY IN RISK ASSESSMENT 9

2.1 Concepts 9

2.2 Application and evaluation 13

2.2.1 Interspecies extrapolation 14

$\begin{array}{ll}\text { 2.2.2 Interindividual variability } & 14\end{array}$

2.2.3 High dose to low dose extrapolation $\quad 15$

$\begin{array}{ll}2.2 .4 \text { Route-to-route extrapolation } & 15\end{array}$

$\begin{array}{ll}\text { 2.2.5 Model evaluation } & 16\end{array}$

3. CHARACTERIZATION AND DOCUMENTATION OF PBPK MODELS 16

3.1 Introduction 16

$\begin{array}{lll}3.2 & \text { Scope and purpose of the model } & 17\end{array}$

$\begin{array}{ll}3.3 \text { Model structure and biological characterization } & 18\end{array}$

3.4 Mathematical description of ADME 19

3.5 Computer implementation and verification 20

$\begin{array}{ll}3.6 & \text { Parameter estimation and analysis } \\ 3.7 & 20\end{array}$

$\begin{array}{ll}\text { 3.7 Model validation and evaluation } & 21\end{array}$

3.7.1 Characterizing the level of confidence in PBPK models 22

3.7.1.1 Biological basis $\quad 22$

3.7.1.2 Comparison of model simulations with data 23

3.7.1.3 Reliability of dose metric predictions (model testing,
uncertainty and sensitivity analyses)

3.7.2 Ability of PBPK models to address PK uncertainty relative to other

$\begin{array}{ll}\text { approaches } & 30 \\ 3.7 .2 .1 \text { Dose metric } & 30\end{array}$

$\begin{array}{ll}3.7 .2 .2 \text { Conceptual model } & 30\end{array}$

$\begin{array}{ll}\text { 3.7.2.3 Input parameters } & 31\end{array}$

$\begin{array}{ll}\text { 3.7.3 Purpose-specific model evaluation } & 31\end{array}$

3.7.3.1 Interspecies extrapolation $\quad 32$

3.7.3.2 Interindividual variability $\quad 34$

3.7.3.3 High dose to low dose extrapolation $\quad 35$

3.7.3.4 Route-to-route extrapolation 36

$\begin{array}{lll}3.8 & \text { Documentation } & 37\end{array}$ 
4. APPLICATION OF PBPK MODELS IN RISK ASSESSMENT 38

$\begin{array}{lll}4.1 & \text { Choice of critical studies } & 38\end{array}$

4.2 Selection of PBPK models $\quad 39$

4.3 Evaluation of dose metrics $\quad 42$

4.4 Determination of human exposures 43

5. PROCESS CONSIDERATIONS 45

5.1 Expertise 45

5.2 Training 46

$\begin{array}{lll}5.3 \text { Communication } & 47\end{array}$

6. CONCLUDING REMARKS 49

REFERENCES $\quad 51$

ANNEX 1: GLOSSARY OF TERMS 63

ANNEX 2: FREQUENTLY ASKED QUESTIONS 68

ANNEX 3: CASE-STUDY ON INTEGRATING PBPK MODELS IN A RISK ASSESSMENT OF A CHEMICAL 


\section{FOREWORD}

Harmonization Project Documents are a family of publications by the World Health Organization (WHO) under the umbrella of the International Programme on Chemical Safety (IPCS) (WHO/ILO/UNEP). Harmonization Project Documents complement the Environmental Health Criteria (EHC) methodology (yellow cover) series of documents as authoritative documents on methods for the risk assessment of chemicals.

The main impetus for the current coordinated international, regional and national efforts on the assessment and management of hazardous chemicals arose from the 1992 United Nations Conference on Environment and Development (UNCED). UNCED Agenda 21, Chapter 19, provides the "blueprint" for the environmentally sound management of toxic chemicals. This commitment by governments was reconfirmed at the 2002 World Summit on Sustainable Development and in 2006 in the Strategic Approach to International Chemicals Management (SAICM). The IPCS project on the Harmonization of Approaches to the Assessment of Risk from Exposure to Chemicals (Harmonization Project) is conducted under Agenda 21, Chapter 19 , and contributes to the implementation of SAICM. In particular, the project addresses the SAICM objective on Risk Reduction and the SAICM Global Plan of Action activity to "Develop and use new and harmonized methods for risk assessment".

The IPCS Harmonization Project goal is to improve chemical risk assessment globally, through the pursuit of common principles and approaches, and, hence, strengthen national and international management practices that deliver better protection of human health and the environment within the framework of sustainability. The Harmonization Project aims to harmonize global approaches to chemical risk assessment, including by developing international guidance documents on specific issues. The guidance is intended for adoption and use in countries and by international bodies in the performance of chemical risk assessments. The guidance is developed by engaging experts worldwide. The project has been implemented using a stepwise approach, first sharing information and increasing understanding of methods and practices used by various countries, identifying areas where convergence of different approaches would be beneficial and then developing guidance that enables implementation of harmonized approaches. The project uses a building block approach, focusing at any one time on the aspects of risk assessment that are particularly important for harmonization.

The project enables risk assessments (or components thereof) to be performed using internationally accepted methods, and these assessments can then be shared to avoid duplication and optimize use of valuable resources for risk management. It also promotes sound science as a basis for risk management decisions, promotes transparency in risk assessment and reduces unnecessary testing of chemicals. Advances in scientific knowledge can be translated into new harmonized methods.

This ongoing project is overseen by a geographically representative Harmonization Project Steering Committee and a number of ad hoc Working Groups that manage the detailed work. Finalization of documents includes a rigorous process of international peer review and public comment. 


\section{LIST OF ACRONYMS AND ABBREVIATIONS}

$\mathrm{AD}_{\mathrm{AF}} \quad$ chemical-specific adjustment factor for interspecies differences in toxicodynamics

ADI acceptable daily intake

ADME

$\mathrm{AD}_{\mathrm{UF}}$ absorption, distribution, metabolism and excretion

$\mathrm{AK}_{\mathrm{AF}}$ default uncertainty factor for interspecies differences in toxicodynamics chemical-specific adjustment factor for interspecies differences in toxicokinetics

$\mathrm{AK}_{\mathrm{UF}}$

AUC default uncertainty factor for interspecies differences in toxicokinetics

$\mathrm{BMC}$ area under the concentration versus time curve

BMCL benchmark concentration

lower confidence limit of the exposure concentration associated with a predetermined response level (e.g. 5\%)

BMD benchmark dose

BMDL lower confidence limit of the dose associated with a predetermined response level (e.g. 5\%)

BW body weight

CSAF chemical-specific adjustment factor

CV coefficient of variation

CYP cytochrome P-450

DNA

GSH

$\mathrm{HD}_{\mathrm{AF}}$

HD UF

$\mathrm{HK}_{\mathrm{AF}}$

$\mathrm{HK}_{\mathrm{UF}}$

IPCS

$K_{\mathrm{m}}$ deoxyribonucleic acid glutathione chemical-specific adjustment factor for human variability in toxicodynamics default uncertainty factor for human variability in toxicodynamics chemical-specific adjustment factor for human variability in toxicokinetics default uncertainty factor for human variability in toxicokinetics International Programme on Chemical Safety

LOAEC Michaelis-Menten constant

LOAEL

MOA lowest-observed-adverse-effect concentration lowest-observed-adverse-effect level mode of action

NOAEC no-observed-adverse-effect concentration

NOAEL PBPK no-observed-adverse-effect level

PBTK

QSAR

PD

PK

POD physiologically based pharmacokinetic physiologically based toxicokinetic quantitative structure-activity relationship pharmacodynamic pharmacokinetic

RfC point of departure

RfD reference concentration

TD toxicodynamic

TDI tolerable daily intake

TK toxicokinetic

VC hypothetical volatile chemical

$V_{\max } \quad$ maximal rate of metabolism 


\section{PREFACE}

This document was prepared through a project on physiologically based pharmacokinetic (PBPK) modelling under the auspices of the World Health Organization (WHO)/International Programme on Chemical Safety (IPCS) Project on the Harmonization of Approaches to the Assessment of Risk from Exposure to Chemicals.

The document content was planned at a meeting of the WHO/IPCS PBPK Planning Group, hosted by the United Kingdom's Health and Safety Laboratory on 5-6 November 2007 in Buxton, England.

The first draft was prepared by Kannan Krishnan, Université de Montréal, Canada, with input from the WHO/IPCS PBPK Planning Group. Woody Setzer and John Wambaugh of the National Center for Computational Toxicology, United States Environmental Protection Agency, Research Triangle Park, NC, United States of America (USA), provided additional input to the first draft.

The draft document was released for public and peer review in September 2008. A second draft was prepared, taking into account comments received, by Kannan Krishnan, with input from the WHO/IPCS PBPK Planning Group.

The second draft document was reviewed and discussed at a WHO/IPCS International Workshop on Principles of Characterizing and Applying PBPK Models in Chemical Risk Assessment, held on 6-8 July 2009, hosted by the German Medical Association (Bundesärztekammer) in Berlin, Germany. Recommendations of the workshop for further development of guidance were considered by the WHO/IPCS PBPK Planning Group in order to prepare the final document. Andy Nong, Health Canada, contributed to the revision of the draft under the guidance of the WHO/IPCS PBPK Planning Group, and the final draft text was reviewed by Kannan Krishnan.

All contributions to the development of the guidance document are gratefully acknowledged.

\section{WHO/IPCS PBPK Planning Group members}

\section{M.E. (Bette) Meek (Chair)}

McLaughlin Centre for Population Health Risk Assessment, University of Ottawa, Ottawa, Ontario, Canada (previously with the Safe Environments Programme, Health Canada, Ottawa, Ontario, Canada)

\section{Hugh A. Barton}

Pharmacokinetics, Dynamics, and Metabolism, Pfizer Global Research and Development, Groton, CT, USA (previously with the National Center for Computational Toxicology, United States Environmental Protection Agency, Research Triangle Park, NC, USA)

\section{Jos Bessems}

Centre for Substances and Integrated Risk Assessment, National Institute for Public Health and the Environment (RIVM), Bilthoven, Netherlands 


\section{Harrie Buist}

Department of Toxicological Risk Assessment, TNO Quality of Life, Zeist, Netherlands

\section{Harvey J. Clewell III}

Center for Human Health Assessment, The Hamner Institutes for Health Sciences, Research

Triangle Park, NC, USA

\section{Ursula Gundert-Remy}

Safety of Substances and Products, Federal Institute for Risk Assessment (BfR), Berlin,

Germany

\section{John C. Lipscomb}

National Center for Environmental Assessment, Office of Research and Development, United States Environmental Protection Agency, Cincinnati, OH, USA

\section{George Loizou}

Computational Toxicology Section, Health and Safety Laboratory, Buxton, England

\section{David Moir}

Systemic Toxicology and Pharmacokinetics Section, Safe Environments Programme, Health Canada, Ottawa, Ontario, Canada

Martin Spendiff (during preparation of the first draft of the document)

Computational Toxicology Section, Health and Safety Laboratory, Buxton, England

\section{Representatives (European Commission: Joint Research Centre)}

George Fotakis (during preparation of the first draft of the document)

European Chemicals Agency (ECHA), Helsinki, Finland (previously with the European Chemicals Bureau, Institute for Health and Consumer Protection, Joint Research Centre, European Commission, Ispra, Italy)

\section{Michel Bouvier-d'Yvoire}

European Commission - DG Entreprise, Unit G1 (REACH), Brussels, Belgium (previously with the Institute for Health and Consumer Protection, Joint Research Centre, European Commission, Ispra, Italy)

\section{Secretariat}

\section{Carolyn Vickers}

International Programme on Chemical Safety, World Health Organization, Geneva, Switzerland 


\section{Berlin workshop participants}

\section{Jos Bessems}

Centre for Substances and Integrated Risk Assessment, National Institute for Public Health and the Environment (RIVM), Bilthoven, Netherlands

\section{Alan Boobis}

Department of Health Toxicology Unit, Hammersmith Campus, Imperial College London, London, England

\section{Susy Brescia}

Health and Safety Executive, Bootle, Merseyside, England

\section{Harrie Buist}

Department of Toxicological Risk Assessment, TNO Quality of Life, Zeist, Netherlands

\section{Weihsueh Chiu}

United States Environmental Protection Agency, Washington, DC, USA

\section{Harvey J. Clewell III}

Center for Human Health Assessment, The Hamner Institutes for Health Sciences, Research Triangle Park, NC, USA

\section{Ivan Dobrev}

Chemical Risk Assessment, Fraunhofer Institute for Toxicology and Experimental Medicine, Hanover, Germany

\section{Ursula Gundert-Remy}

Safety of Substances and Products, Federal Institute for Risk Assessment (BfR), Berlin, Germany

\section{Wolfgang Heger (Day 1 only)}

Federal Environment Agency, Berlin, Germany

\section{Gunnar Johanson}

Institute of Environmental Medicine, Karolinska Institutet, Stockholm, Sweden

\section{George Kowalczyk}

Greater Manchester Health Protection Unit, Health Protection Agency, Manchester, England

\section{John C. Lipscomb}

National Center for Environmental Assessment, Office of Research and Development, United States Environmental Protection Agency, Cincinnati, OH, USA

\section{Manupat Lohitnavy}

Department of Pharmacy Practice, Faculty of Pharmaceutical Sciences, Naresuan University, Phitsanulok, Thailand

\section{George Loizou}

Computational Toxicology Section, Health and Safety Laboratory, Buxton, England 


\section{M.E. (Bette) Meek}

Chemical Risk Assessment, McLaughlin Centre for Population Health Risk Assessment, University of Ottawa, Ottawa, Ontario, Canada

\section{Hans Mielke}

Federal Institute for Risk Assessment (BfR), Berlin, Germany

\section{David Moir}

Systemic Toxicology and Pharmacokinetics Section, Safe Environments Programme, Health Canada, Ottawa, Ontario, Canada

\section{Andy Nong}

Exposure and Biomonitoring Division, Environmental Health, Science and Research Bureau, Health Canada, Ottawa, Ontario, Canada

\section{Susan Rieth}

Integrated Risk Information System (IRIS) Program, United States Environmental Protection Agency, Washington, DC, USA

\section{R. Woodrow Setzer}

National Center for Computational Toxicology, United States Environmental Protection Agency, Research Triangle Park, NC, USA

\section{Dick Sijm}

REACH Bureau, Expertise Centre for Substances, National Institute for Public Health and the Environment (RIVM), Bilthoven, Netherlands

\section{Marco Zeilmaker}

National Institute for Public Health and the Environment (RIVM), Bilthoven, Netherlands

\section{Representatives and industry}

European Commission: European Chemicals Agency

George Fotakis

Helsinki, Finland

\section{European Commission: Joint Research Centre}

\section{Michel Bouvier-d'Yvoire}

Chemical Assessment and Testing Unit, Institute for Health and Consumer Protection, Joint Research Centre, European Commission, Ispra, Italy

European Food Safety Authority

Jean-Lou Dorne

Parma, Italy

Industry

Hugh A. Barton

Pharmacokinetics, Dynamics, and Metabolism, Pfizer Global Research and Development, Groton, CT, USA 


\section{Secretariat}

\section{Kannan Krishnan}

Consultant, Pierrefonds, Quebec, Canada

\section{Carolyn Vickers}

International Programme on Chemical Safety, World Health Organization, Geneva, Switzerland 


\section{INTRODUCTION}

\subsection{Context}

This document provides guidance on the characterization and application of physiologically based pharmacokinetic (PBPK) or physiologically based toxicokinetic (PBTK) ${ }^{1}$ models in risk assessment. PBPK models are quantitative descriptions of the absorption, distribution, metabolism and excretion (ADME) of chemicals in biota based on interrelationships among key physiological, biochemical and physicochemical determinants of these processes. They are part of the broader continuum of increasingly data-informed approaches, ranging from the commonly adopted default based on external dose to more biologically realistic doseresponse models. By facilitating the incorporation of internal dose measures of relevance to the mode of action (MOA) by which a chemical is hypothesized to cause toxicity (normally critical effects), PBPK models facilitate more scientifically sound extrapolations across studies, species, routes and dose levels. They are also fundamental to the development of biologically based dose-response models to address uncertainty and variability related to TK and TD.

This document represents the product of an initiative undertaken as part of the International Programme on Chemical Safety (IPCS) Project on Harmonization of Approaches to the Assessment of Risk from Exposure to Chemicals. It complements the outputs of other initiatives of the IPCS Harmonization Project, particularly those relating to the weight of evidence for MOA of chemicals, human exposure models and chemical-specific adjustment factors (CSAFs) (IPCS, 1999, 2004, 2005a,b, 2008; Sonich-Mullin et al., 2001; Meek et al., 2003b; Boobis et al., 2006, 2008). A specific focus of this document is on promoting best practices in characterizing and applying PBPK models, to facilitate greater usage of these tools by risk assessors.

This IPCS document draws upon the output of two international workshops that addressed specific technical issues in PBPK modelling relevant to enhancing their uptake in risk assessment:

- the International Workshop on Uncertainty and Variability in PBPK Models, held at Research Triangle Park, North Carolina, USA, on 31 October - 2 November 2006, sponsored by the United States Environmental Protection Agency (National Center for Environmental Assessment, National Center for Computational Toxicology and National Health Effects and Environmental Research Laboratory, Office of Research and Development); National Institute of Environmental Health Sciences, National Institutes of Health, USA; and National Toxicology Program, USA;

- the International Workshop on the Development of Good Modelling Practice for PBPK Models, held at the Mediterranean Agronomic Institute of Chania, Crete, Greece, on 2628 April 2007, sponsored by the United Kingdom Health and Safety Executive, the United Kingdom Health and Safety Laboratory, Health Canada, the European Chemical Industry Council and the European Centre for the Validation of Alternative Methods.

\footnotetext{
${ }^{1}$ In this document, the terms "pharmacokinetics" (PK) and "toxicokinetics" (TK) are considered to have the same meaning. Therefore, "physiologically based pharmacokinetic model" is equivalent to "physiologically based toxicokinetic model". Similarly, the term "toxicodynamics" (TD) is interpreted as the same as "pharmacodynamics" (PD) in this report (IPCS, 2005a).
} 
Additionally, capitalizing on recent, separate initiatives within the USA, Canada and Europe, this IPCS document provides 1) an international perspective that seems to be lacking so far, 2) a basis for international endorsement of the best practices for characterizing and applying PBPK models in risk assessment and 3) a means of raising the level of awareness on the applicability and value of PBPK modelling in risk assessment.

\subsection{Objectives}

The primary goal of this report is to document the key principles and best practices for characterizing and applying PBPK models in risk assessment. This report presents these principles both for risk assessors who need to evaluate PBPK models for use in risk assessment as well as for PBPK modellers who are interested in developing models capable of risk assessment applications. There is a particular emphasis on the need for effective and transparent communication to facilitate greater usage of PBPK models by the risk assessment community. In this regard, examples and a case-study dealing with the characterization and application of PBPK models in risk assessment are presented and discussed throughout this document.

\subsection{Organization}

The main text of this guidance document is organized in five sections. The current section presents a brief historical account of the process that went into the development of this document, as well as contextual reference of this guidance to other initiatives of the IPCS Project on the Harmonization of Approaches to the Assessment of Risk from Exposure to Chemicals. Section 2 addresses the critical role of TK and dosimetry modelling in risk assessment, whereas section 3 summarizes the principles for characterizing and documenting PBPK models intended for use in risk assessments. Section 4 discusses the principles and practice of PBPK model application in health risk assessment, with particular reference to interspecies, interindividual and route-to-route extrapolations. Section 5 focuses on process considerations regarding the incorporation of PBPK models within risk assessment; specific aspects discussed in this section include training, expertise and communication issues to overcome the impediments to the use of PBPK models in risk assessment.

Appended to this document are a glossary of terms (Annex 1), a list of frequently asked questions regarding PBPK modelling (Annex 2) and a template for PBPK model documentation containing critical information for description and application in a risk assessment context, followed by illustration with a specific example using a hypothetical chemical "VC" (Annex 3).

\section{TISSUE DOSIMETRY IN RISK ASSESSMENT}

\subsection{Concepts}

Health risk assessments are conducted on the basis of problem formulation, hazard identification, dose-response assessment, exposure assessment and risk characterization (NRC, 1983; IPCS, 1999, 2005a, 2008). The dose-response assessment frequently involves the identification of a point of departure (POD) for deriving the acceptable external exposure concentration or tolerable daily dose for humans, including sensitive individuals. In the case 
of threshold toxicants, the POD, such as the no-observed-adverse-effect level (NOAEL), the no-observed-adverse-effect concentration (NOAEC), the lowest-observed-adverse-effect level (LOAEL), the lowest-observed-adverse-effect concentration (LOAEC), the benchmark dose (BMD), the benchmark concentration (BMC), the lower confidence limit on the benchmark dose (BMDL) or the lower confidence limit on the benchmark concentration (BMCL), is divided by numerical factors to determine the tolerable daily intake (TDI), acceptable daily intake (ADI), reference dose (RfD) or reference concentration (RfC) (IPCS, 1994, 1999, 2004, 2009). These factors are variously referred to as assessment factors, uncertainty factors, safety factors or extrapolation factors by various jurisdictions (Vermeire et al., 1999; IPCS, 2004; Ritter et al., 2007; Konietzka et al., 2008). Non-threshold toxicants, particularly for substances that interact with genetic material, are characterized by various methods, including health risk estimates, effective doses or margins of exposure (Younes et al., 1998; IPCS, 2005a).

Refinements in risk assessment can be based upon additional scientific data that can be used as a basis to estimate internal exposure dose or concentration (Figure 1). This information can help to better approximate differences in sensitivities between test animals and humans and between routes of exposure and induced harm at low doses over long periods of exposure. For example, data on physicochemical characteristics or species-specific considerations can be applied in "categorical approaches" to better inform interspecies extrapolation, interindividual variability and route-to-route extrapolations (e.g. Jarabek, 1994; Naumann et al., 2001). If there are adequate chemical-specific kinetic or dynamic data, they can be incorporated to better characterize interspecies differences and human variability in doseresponse assessment (IPCS, 2005a). The IPCS framework for CSAFs, based on replacement of TK and TD components of default uncertainty factors, enables the risk assessor to consider and incorporate data on MOA with the incentive of using a more appropriate assessment factor in risk assessments instead of the default values (i.e. presumed health protective) (Figure 2).

Use of the external dose for PODs is essentially uninformative of the dose metric that is closely and directly related to adverse responses. Therefore, a default external dose value does not necessarily provide a scientifically sound basis to extrapolate to other scenarios, species, individuals or exposure routes (Clewell et al., 2002). This approach is particularly uncertain for complex dose metric relationships, such as those involving non-linearity between exposure and target dose metrics or saturable kinetics of the parent compound or metabolites. In these cases, the application and exploration of PK data enable such differences between external and internal dose metrics to be addressed.

PK or TK studies determine the fate of a chemical in the body based on the rate of absorption into the body, distribution and storage in tissues, metabolism and excretion. These PK processes are incorporated into a mathematical model structure on the basis of interplay among critical physiological (e.g. body weight or blood flows), physicochemical (e.g. tissue and blood partitioning) and biochemical (e.g. liver metabolic or urinary excretion rates) characteristics of a chemical. The models are not intended to precisely characterize the PK processes but represent an interpretation of the available data by addressing the relationships between an external dose and internal tissue, blood or excretion dose. For this reason, supported by scientific evidence, biochemical and physiological assumptions based on differences among species, individuals, dose levels and durations can be rationalized into the model. The motivation in using PK models, particularly PBPK models, and tissue dosimetry 
Basic data set

\begin{tabular}{|c|c|c|}
\hline EXTERNAL \\
\cline { 3 - 3 }
\end{tabular}

Kinetic parameters

\begin{tabular}{|c|c|c|}
\hline $\begin{array}{c}\text { EXTERNAL } \\
\text { DOSE }\end{array}$ & $\begin{array}{l}\text { INTERNAL } \\
\text { DOSE }\end{array}$ & $\begin{array}{c}\text { TOXIC } \\
\text { RESPONSE }\end{array}$ \\
\hline
\end{tabular}

Physiologically based pharmacokinetic (PBPK) models

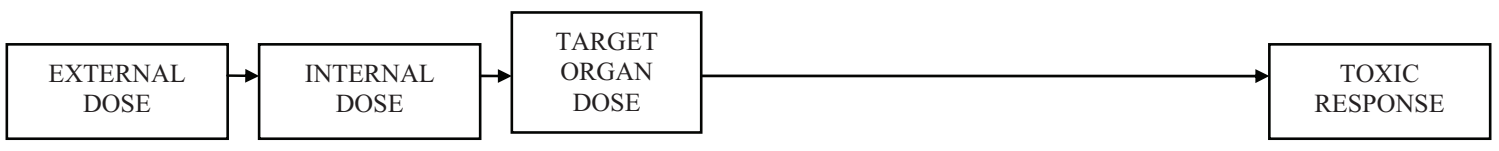

PBPK models with target organ metabolism

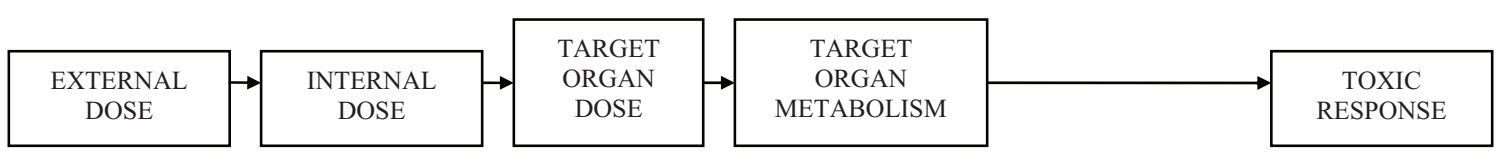

Biologically based dose-response model

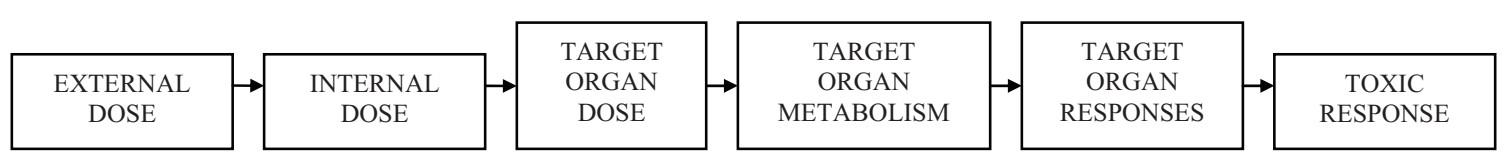

Figure 1: The relationship between external dose and toxic response for an increasingly datainformed dose-response analysis (based on Renwick et al., 2001; IPCS, 2005a).

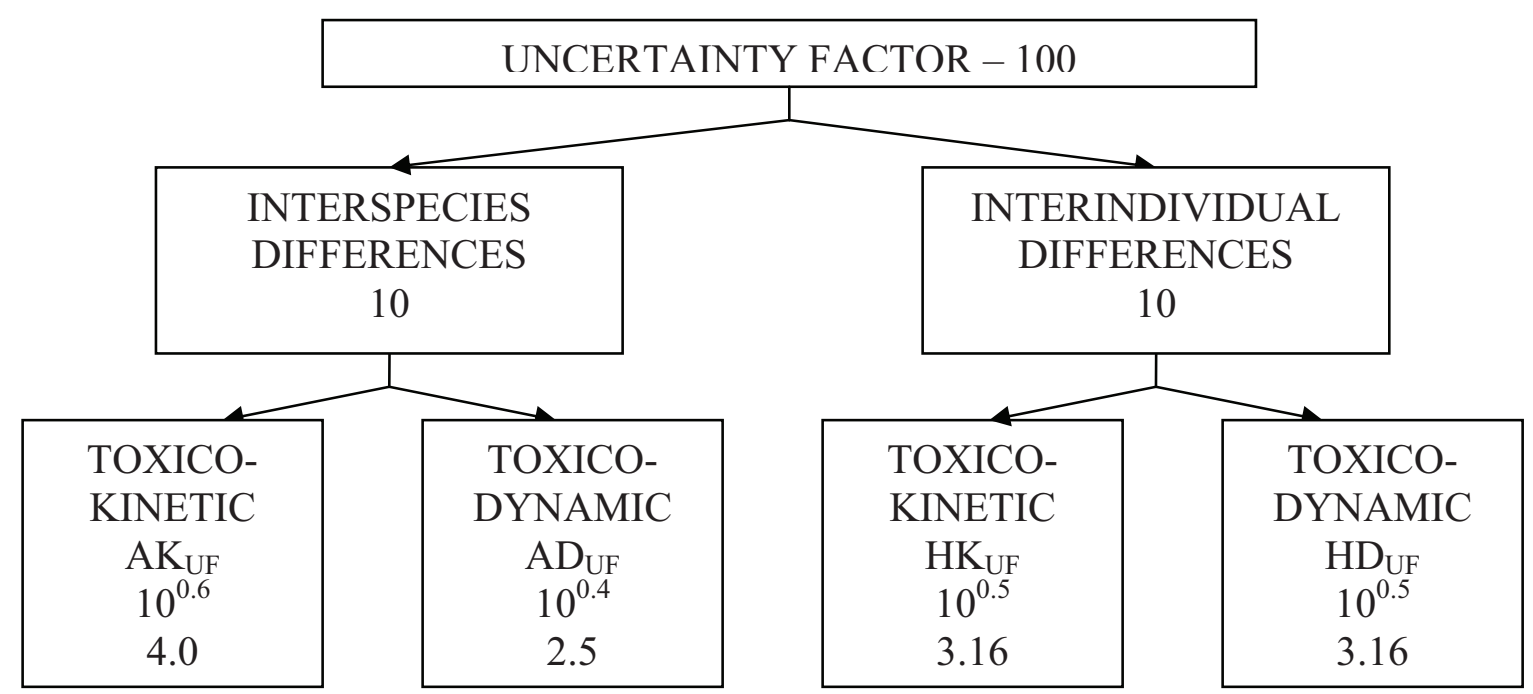

Figure 2: Subdivision of the composite uncertainty factor (adapted from IPCS, 2005a). AK default uncertainty factor for interspecies differences in toxicokinetics; $A D_{U F}$, default uncertainty factor for interspecies differences in toxicodynamics; $\mathrm{HK}_{\mathrm{UF}}$, default uncertainty factor for human variability in toxicokinetics; $\mathrm{HD}_{\mathrm{UF}}$, default uncertainty factor for human variability in toxicodynamics. 


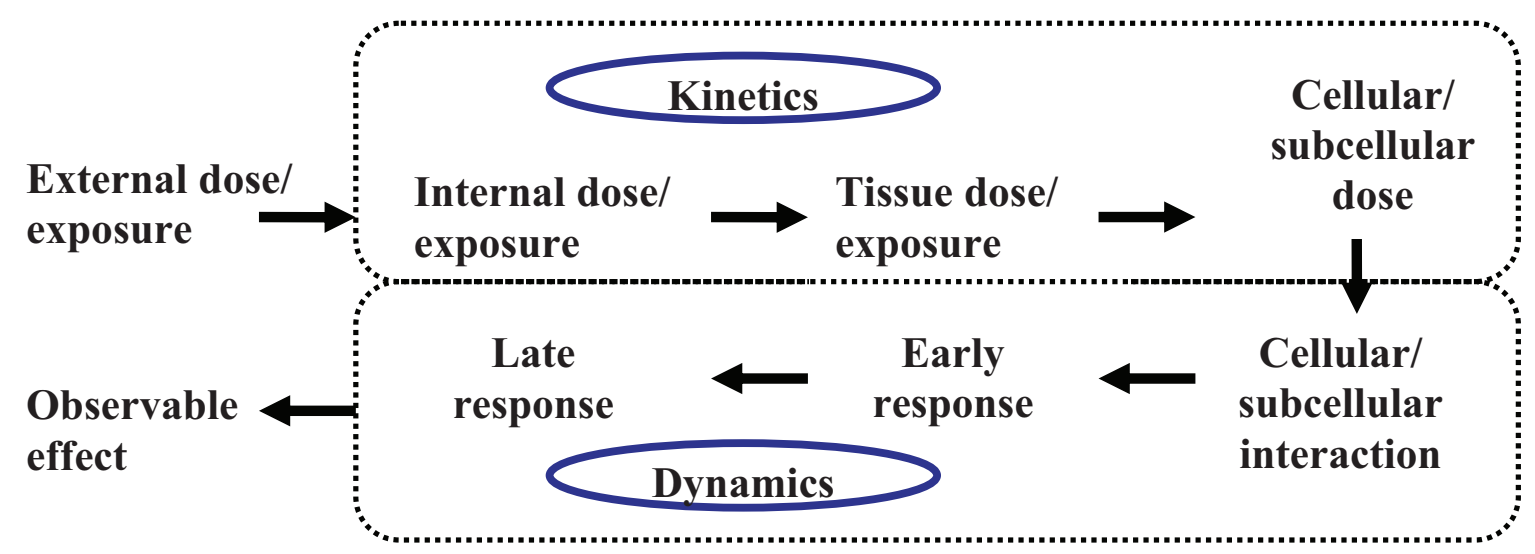

Figure 3: Schematic of the processes involved in the expression of toxic responses placed in a risk assessment context.

concepts in risk assessment is essentially to provide a better representation of "biologically effective dose" in lieu of external exposure.

The risk assessment process has evolved over the years to effectively accommodate increasingly data-informed approaches, particularly for chemicals with a critical exposureresponse characteristic. In such cases, the tendency is to move away from the conduct of "external dose versus response" analysis and incorporate relevant data on MOA, including TK and TD, when the data are adequate to do so. In this context, MOA refers to a series of key events leading to the induction of relevant toxicity end-points for which weight of evidence supports plausibility (IPCS, 2005a). For a given chemical, the MOA reflects the current state of knowledge of the biological basis of the response and the relevant "dose metric" (i.e. the dose measure that is causally related to the toxic outcome) (Andersen et al., 1987; Clewell et al., 2002; Andersen, 2003). The application of PBPK modelling for doseresponse analysis offers a more accurate extrapolation to human exposure conditions by providing an analysis based on target tissue or cellular/subcellular dose (Figure 3). Implicit in such an application is the assumption that the toxic effects in the target tissue are closely related to the concentration of the active form of the substance, consistent with an understanding of the MOA underlying the adverse response (Clewell et al., 2002). For an MOA-oriented risk assessment, scientific evidence (including anatomical, physiological, biochemical and physicochemical data) can be incorporated into PBPK models to provide a representation of dose metrics relevant to a biological response.

The organization of MOA, biochemical, physiological and PK data into a structured PBPK model provides the risk assessor with an alternative option to describe dose metrics that are more relevant to dose outcome (Table 1). Internal (tissue) doses of chemicals have been increasingly interpreted with PBPK models as a means to address the difference between species, routes and dose-dependent kinetics beyond the scope of an external dose (Clewell \& Andersen, 1985, 1987; Clewell et al., 2002; Clark et al., 2004; Chiu et al., 2007; Loizou et al., 2008; Thompson et al., 2009). The PBPK models have also been used to extrapolate within life stages (Clewell et al., 2004; Yoon \& Barton, 2008; Verner et al., 2009), as well as to address variability among individuals in a population (Bois, 2001; Hack et al., 2006; Barton et al., 2007). These applications are possible by combining various sources of information, such as in vivo and in vitro data, experimental animal and human physiological information, or population phenotypic and metabolic measurements. With their potential being recognized and application increasing, harmonization and guidance in the development and documentation of PBPK models as well as establishing criteria of confidence and applicability have 
been recognized as important steps towards increasing acceptance in their use for internal dosimetry estimation (Loizou et al., 2008).

Table 1: Risk assessment options for exposure dose estimation based on the available scientific information for the test animal to human extrapolation aspect.

\begin{tabular}{|c|c|c|}
\hline Options & Process & Needs \\
\hline $\begin{array}{l}\text { Conventional (default) } \\
\text { approaches }\end{array}$ & $\begin{array}{l}\text { Divide animal POD by uncertainty } \\
\text { factor, or scale it on the basis of body } \\
\text { weight }\end{array}$ & No data or model required \\
\hline CSAF approach & $\begin{array}{l}\text { Divide animal POD by CSAF derived } \\
\text { from empirical data, PK parameters } \\
\text { or PBPK models }\end{array}$ & $\begin{array}{l}\text { Categorical or chemical-specific PK } \\
\text { data/models }\end{array}$ \\
\hline PBPK analysis & $\begin{array}{l}\text { Translate animal POD to internal } \\
\text { dose metric using animal PBPK } \\
\text { model; compute human-equivalent } \\
\text { dose (or concentration) using human } \\
\text { PBPK model }\end{array}$ & $\begin{array}{l}\text { Animal and human PBPK models; } \\
\text { limited PK data; characterization of } \\
\text { the relationship between dose metric } \\
\text { and response }\end{array}$ \\
\hline
\end{tabular}

\subsection{Application and evaluation}

A major advantage of PBPK models over empirical compartmental descriptions is greater extrapolation power. When fundamental biochemical processes are described, extrapolation over dose ranges where saturation of metabolism occurs is possible (Ramsey \& Andersen, 1984; Clewell \& Andersen, 1985, 1987; Krishnan \& Andersen, 2007). Similarly, PBPK models are instrumental in facilitating the prediction of interindividual, interspecies and route differences in dose metrics based on physiological and physicochemical properties. For example, interspecies extrapolation is conducted either by replacing input parameter values (e.g. tissue blood flow rates and tissue volumes) specific to the species of interest (e.g. Arms \& Travis, 1988; Davies \& Morris, 1993; Brown et al., 1997) or by estimating them on the basis of body surface area or body weight differences (Adolph, 1949; Dedrick et al., 1973; Dedrick \& Bischoff, 1980).

PBPK models are essentially intended to estimate target tissue dose in species and under exposure conditions for which few or no data exist. If a complete PK data set were available for the exposure scenarios and species of interest, there would be no need to develop a PBPK model. An optimal PK data set for risk assessment would consist of the time course data on the most appropriate dose metric associated with exposure scenarios and doses used in the critical studies selected for the assessment (e.g. laboratory animal bioassays or clinical and epidemiological studies) and relevant human exposure conditions. In the absence of such information, it is possible to explore and integrate information from the current knowledge base of a chemical and fundamental biology (from animal, metabolic or in vitro studies) within a PBPK model to better quantify the impact in humans, through estimation of internal dose (Clewell \& Andersen, 1985; NRC, 1987; Gerrity \& Henry, 1990; Krishnan et al., 2002; Tan et al., 2007). This approach provides a risk assessor with an opportunity for conducting interspecies, intraspecies, high dose to low dose and route-to-route extrapolations of chemicals present singly or as mixtures with the most appropriate level of confidence, where data are limited. 


\subsubsection{Interspecies extrapolation}

In the absence of cross-species information, the extrapolation of exposure dose corresponding to a POD from one species to another (typically from test animal species to humans) has been addressed with the use of an uncertainty factor of 10, body surface area scaling or body weight (BW) raised to a fractional power ${ }^{2}$ (Andersen et al., 1995b; Dourson et al., 1996; Jacobs, 2007). These approaches are assumed to provide PK-equivalent doses in test species and humans and for certain chronic exposure conditions: 1) the biologically active form is the parent chemical, 2) the clearance and TD processes are proportional to body surface area in both test animal species and humans and 3) the area under the blood concentration versus time curve (AUC) is the appropriate surrogate measure of target tissue dose. When one or more of these assumptions do not hold, these default approaches may not be sufficiently health protective or may be overly conservative; consequently, the adequacy of these approaches to account for interspecies differences in target tissue dose and tissue response for specific chemicals may be verified using chemical-specific data or PK models (Andersen et al., 1991, 1995b; Kirman et al., 2003).

Subdivision of the interspecies uncertainty factor of 10 into TK and TD components would allow part of the default value to be replaced by chemical-specific data consistent with the weight of evidence for MOA when they are available. Accordingly, the default uncertainty factor for interspecies differences in toxicodynamics $\left(A D_{U F}\right)$ of 2.5 (equal to $10^{0.4}$ ) and the default uncertainty factor for interspecies differences in toxicokinetics $\left(\mathrm{AK}_{\mathrm{UF}}\right)$ of 4 (equal to $10^{0.6}$ ) were proposed on the basis of observations and data reviewed by Renwick (1993, 1999) (see Figure 2). An even split between the two components was not favoured based on the greater potential for differences in tissue dose than for differences in tissue response between experimental animals and humans (Renwick, 1993; Walton et al., 2001a,b,c, 2004). The proposed default values of 4 and 2.5 for the TK and TD components, however, are reflective of the rat as the test animal species. Therefore, the use of these values for other test species (e.g. mice, dogs) would increase uncertainty. As such, when relevant species-specific and chemical-specific TK and TD data are available, the default values of $\mathrm{AD}_{\mathrm{UF}}$ and $\mathrm{AK}_{\mathrm{UF}}$ can be replaced with CSAFs for interspecies differences in TK and TD (i.e. the CSAF for interspecies differences in $\mathrm{TK}\left[\mathrm{AK}_{\mathrm{AF}}\right]$ and the CSAF for interspecies differences in $\mathrm{TD}$ $\left[\mathrm{AD}_{\mathrm{AF}}\right]$ ) (Health Canada, 1994; Renwick, 1999; Gundert-Remy \& Sonich-Mullin, 2002; Meek et al., 2003a; IPCS, 2005a). More importantly, $\mathrm{AK}_{\mathrm{AF}}$ can be informed through the use of PBPK models, which facilitate the conduct of interspecies (i.e. test species to human) extrapolation of exposure doses on the basis of target organ dose or another dose metric closely related to the toxic response (Rowland, 1985; Andersen et al., 1995b; Jarabek, 1995). In that situation, a dose metric value would be directly determined from model prediction as a basis to replace the default. Replacement of TK default values for interspecies differences is based on ratios of mean values for the relevant dose metrics in the experimental species versus humans.

\subsubsection{Interindividual variability}

The application of an assessment factor to account for human variability is based on the following assumptions (Dourson et al., 1996; Price et al., 1999):

${ }^{2} \mathrm{BW}^{0.67}$ to $\mathrm{BW}^{0.75}$. 
- variability in target organ dose and toxicological response in a population is greater than that observed in a finite study sample; and/or

- certain individuals and conditions in the human population (e.g. neonates, elderly, pregnant women, diseased, polymorphic) are more sensitive than the average healthy individual owing to differences in target tissue dose or tissue response.

Several jurisdictions have used an assessment or uncertainty factor ranging from 1 to 10 (reviewed in Dourson et al., 1996; Vermeire et al., 1999), implying that the exposure dose associated with a certain level of response (or the NOAEL) could vary between the "average" individual and "sensitive" individuals by as much as an order of magnitude. The applicability of the default value of 10 for all chemicals, regardless of the extent of available information on the population variability in TK, TD or MOA, lacks a clearly defined scientific basis. It is uncertain, then, as to whether the default value is adequate, inadequate or overly conservative for specific chemicals. In this regard, when relevant data on PK and PD are available, the IPCS framework for the development of CSAFs facilitates the rational development of a CSAF, thus allowing deviation from the default approach. Based on a strategy similar to that for interspecies considerations, IPCS suggested a value of 3.16 as the default uncertainty factor for human variability in toxicokinetics $\left(\mathrm{HK}_{\mathrm{UF}}\right)$ and a value of 3.16 as the default uncertainty factor for human variability in toxicodynamics $\left(\mathrm{HD}_{\mathrm{UF}}\right)$, which can in turn be modified (i.e. the CSAF for interindividual variability in $\mathrm{TK}\left[\mathrm{HK}_{\mathrm{AF}}\right]$ and the CSAF for interindividual variability in $\mathrm{TD}\left[\mathrm{HD}_{\mathrm{AF}}\right]$ ) according to the availability of $\mathrm{PK}$ and $\mathrm{PD}$ data (IPCS, 2005a). With the availability of PBPK models, more relevant data (i.e. dose metrics in a population, including specific life stages and subgroups) can be incorporated into risk assessments for a scientifically sound characterization of human variability in PK (Barton et al., 1996; Clewell et al., 1999; Haber et al., 2002; Lipscomb \& Ohanian, 2007). Replacement of default for this component of uncertainty must necessarily characterize variability in the human population and is commonly based on ratios of percentiles to mean values for the relevant dose metric.

\subsubsection{High dose to low dose extrapolation}

The extrapolation of tissue dose and tissue response from high dose to low dose is particularly important when toxicity data are collected in laboratory animals. The doseresponse relationship, based on exposure dose, is frequently complex due to non-linearity in some TK processes (Slikker et al., 2004). For example, the target tissue dose of a toxic moiety is not likely to be proportional to an administered dose in animal bioassays when saturable metabolic pathways are operative in that dose range (e.g. Gehring et al., 1978). In such cases, the derivation of cancer risk or POD based on an appropriate measure of internal dose-as estimated by PBPK models - would be scientifically sound and more closely related to tissue response than the exposure dose (e.g. Andersen et al., 1987).

\subsubsection{Route-to-route extrapolation}

Often the toxicity data or POD for a given chemical might be available for one exposure route (e.g. oral), whereas other exposure routes (e.g. dermal, inhalation) might also be relevant for risk assessment. In such cases, route-to-route extrapolation is conducted if the toxicity of the compound is systemic and not local (i.e. portal of entry) (ECETOC, 2003; IGHRC, 2006). Depending upon the extent of available data, such extrapolations have been conducted on the basis of equivalent exposure dose or absorbed dose (e.g. Gerrity \& Henry, 1990; Pauluhn, 2003). These approaches, however, do not facilitate the derivation of route- 
specific exposure doses on the basis of equivalent tissue exposure or tissue response, because they do not account for route-to-route differences in first-pass effect, PK processes or dose metrics of relevance to the MOA in the test animal species and humans (ECETOC, 2003; IGHRC, 2006). Such considerations to address differences related to route-to-route extrapolation for the same species are facilitated by PBPK models (Clewell \& Andersen, 1987; Gerrity \& Henry, 1990).

\subsubsection{Model evaluation}

For risk assessment application, the model should be able to simulate the dose metrics of relevance to the MOA of the chemical. Finally, it needs to be fit for purpose; in other words, it should have the essential features (i.e. in terms of structure and parameters) consistent with the intended use in risk assessment (e.g. high dose to low dose extrapolation).

The key aspects of characterization and documentation of PBPK models are discussed in section 3, whereas aspects relating to the applicability of PBPK models in risk assessment are considered in section 4 .

\section{CHARACTERIZATION AND DOCUMENTATION OF PBPK MODELS}

\subsection{Introduction}

PBPK models provide quantitative descriptions of ADME of chemicals in biota based on the interrelationships among physiological, biochemical and physicochemical determinants of these processes (Teorell, 1937a,b; Dedrick et al., 1972; Fiserova-Bergerova, 1983; NRC, 1987; Andersen, 2003; Reddy et al., 2005; Lipscomb \& Ohanian, 2007). Figure 4 presents the key steps involved in the development of PBPK models. Typically, the development of PBPK models intended for risk assessment application would begin with a critical evaluation of the available information to assemble the following chemical-specific or more generic (i.e. species-specific) information:

- the nature of the critical effect;

- the dose-response relationship for the critical effect;

- the MOA, including the nature of the toxic moiety (i.e. whether the parent chemical, a stable metabolite or a reactive intermediate produced during metabolism is responsible for the toxicity);

- the pathways (e.g. routes of excretion) and rates (e.g. metabolic rates) of ADME; and

- the physiology (i.e. tissue weights and blood flow rates) of the species of interest.

Several authors (Andersen et al., 1995a; Kohn, 1995; Clark et al., 2004; Gentry et al., 2004; Barton et al., 2007; Chiu et al., 2007; Clewell \& Clewell, 2008; Loizou et al., 2008) have discussed good PBPK modelling principles and practices in terms of the following aspects:

- $\quad$ scope and purpose of the model;

- model structure and biological characterization;

- mathematical description of ADME; 


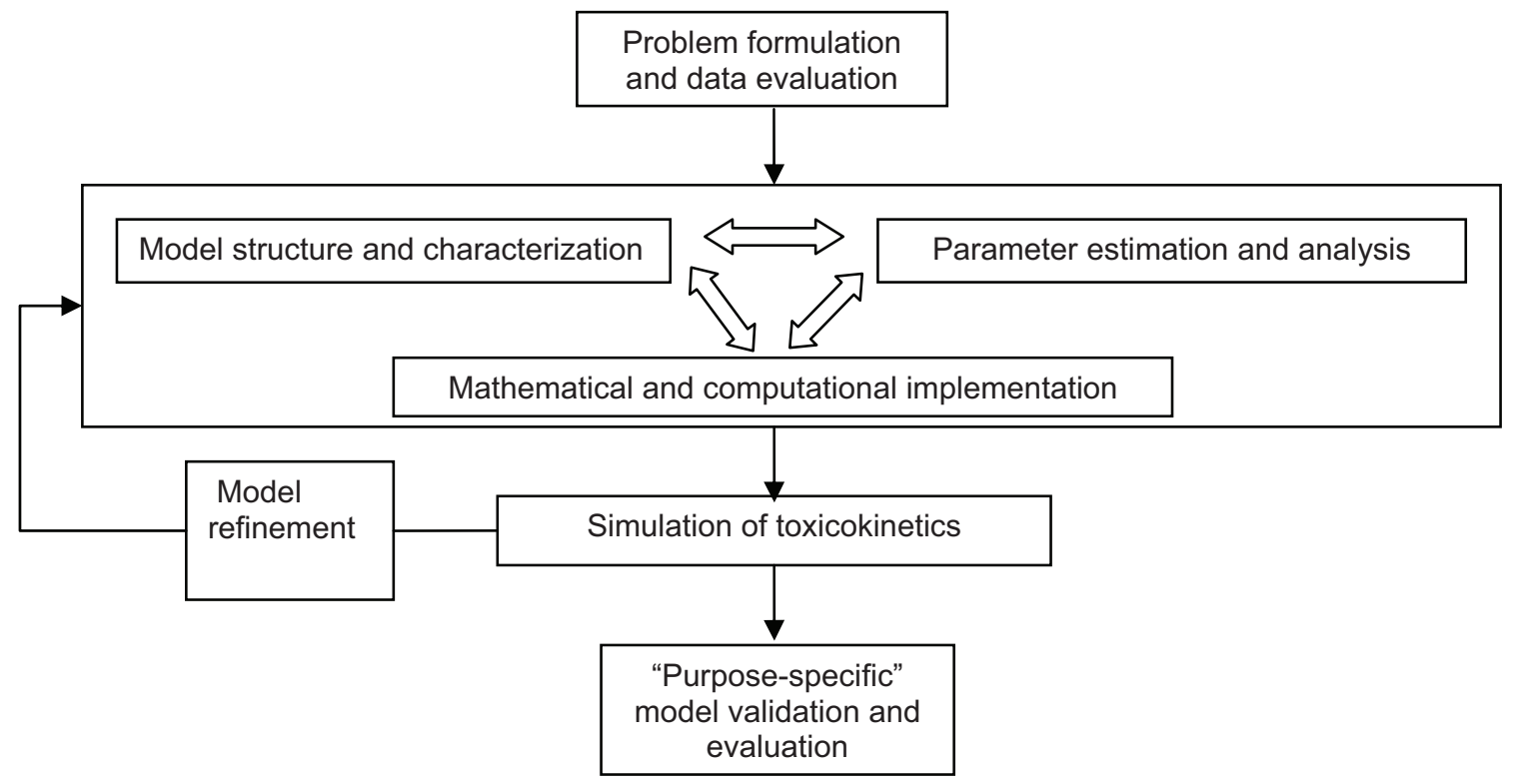

Figure 4: Schematic of the steps involved in the development of PBPK models for application in risk assessment.

- computer implementation and verification;

- parameter estimation and analysis; and

- model validation and evaluation.

Building upon these principles, the following sections highlight the key aspects of characterization and documentation of PBPK models intended for use in risk assessment.

\subsection{Scope and purpose of the model}

The scope for the use of a PBPK model in a particular risk assessment essentially determines the intended model capability and the extent of model evaluation (Box 1). Therefore, it is critical to clearly identify the type of risk assessment it is intended to support (e.g. screening or full; cancer or non-cancer; acute or chronic), the aspects of the assessment it is designed to facilitate (e.g. cross-route or cross-species dosimetry), as well as the MOA hypotheses and associated weight of evidence underlying the model structure (e.g. toxicity from a reactive metabolite versus receptor binding). In each case, then, the strategy for incorporating relevant data (i.e. PBPK model results) to address or resolve the sources of uncertainty in a risk assessment would determine the extent of the modelling effort. For example, if the uncertainty in a current or proposed risk assessment for a given chemical relates to lack of knowledge regarding interspecies differences in tissue dosimetry, a PBPK model capable of providing human-equivalent doses would be sought. A single PBPK model may not be sufficient to address all areas of uncertainty (e.g. a PBPK model in an adult animal may not be adequate to simulate kinetics during specific windows of susceptibility during gestation). Therefore, the purpose and capability of PBPK models should be characterized in terms of the species, life stage, exposure routes/windows and dose metrics that are central to their application in risk assessment (Box 1). 


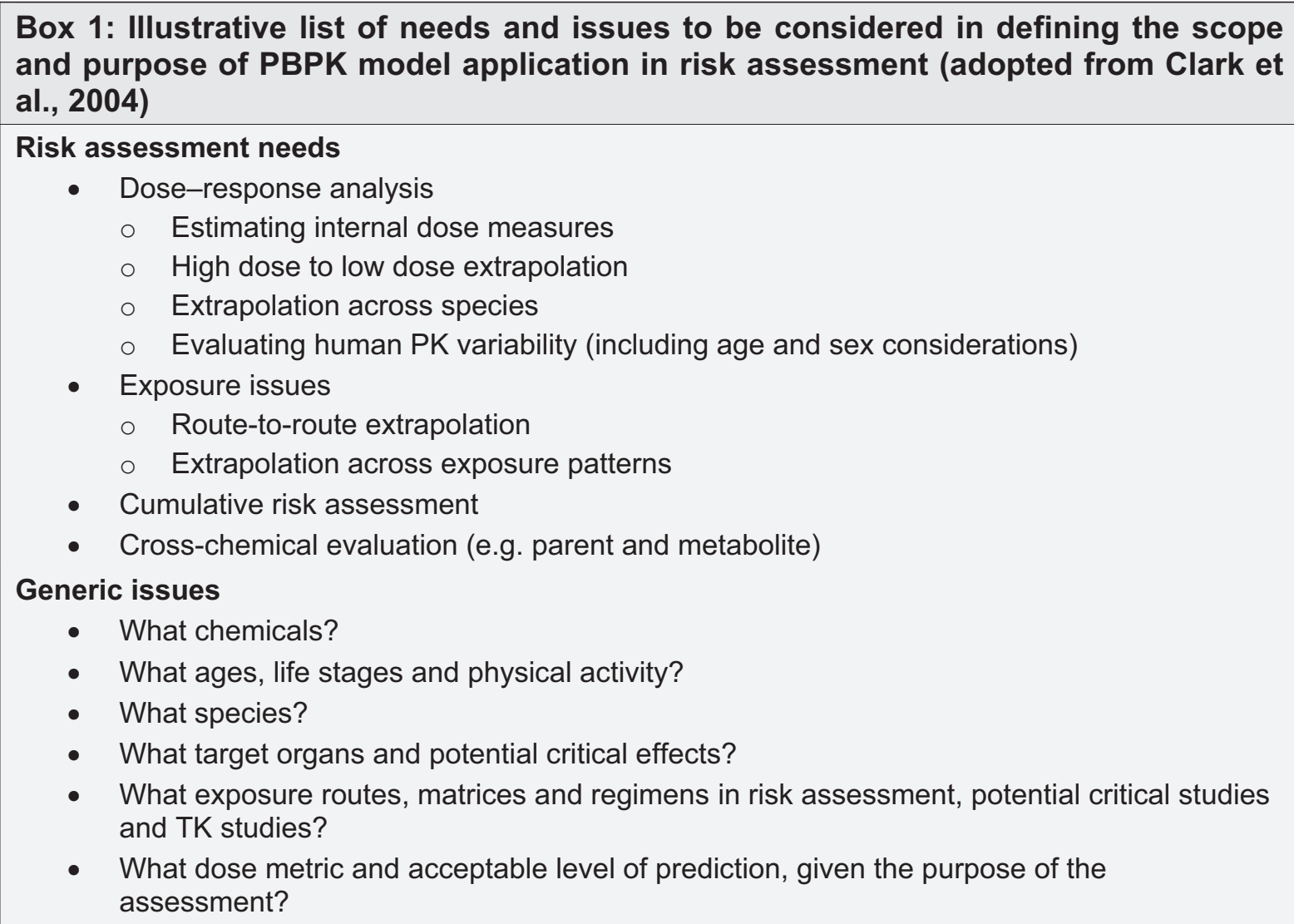

\subsection{Model structure and biological characterization}

The structure of a PBPK model should be characterized in the form of boxes and arrows; the organs and organ systems are represented by the boxes, and the specific physiological or clearance processes are identified by the arrows (e.g. Figure 5). The model structure should reflect a balance between the principles of parsimony (i.e. minimal but essential elements characterizing a system) and plausibility (i.e. reflective of physiological reality and consistent with current state of knowledge) in order to simulate dose metrics of relevance to risk assessment. The need to represent a particular organ or tissue as a separate compartment (either by lumping or by splitting) is determined based on its relevance to target organ toxicity, MOA, TK mechanisms and portal of entry (exposure route) of the chemical under study (Krishnan \& Andersen, 2007). For a hydrophilic chemical whose concentrations are fairly similar across compartments, a one-compartment model might be sufficient. However, for chemicals exhibiting different time course profiles in various tissues, a multicompartmental description is required. Typically, tissues exhibiting similar concentration versus time course behaviour are lumped together (e.g. richly perfused tissues, poorly perfused tissues). However, model complexity and the number of compartments should not be equated with accuracy and usefulness of the model description; model complexity and capability should be consistent with the intended purpose and underlying data. In this regard, the initial version of a PBPK model for organic chemicals has often been based on a so-called "generic" model (e.g. Ramsey \& Andersen, 1984; Brightman et al., 2006) consisting of descriptions of the major ADME processes and mechanisms. Deviations from such generic structures are then characterized on the basis of specific mechanisms of ADME of a chemical. 


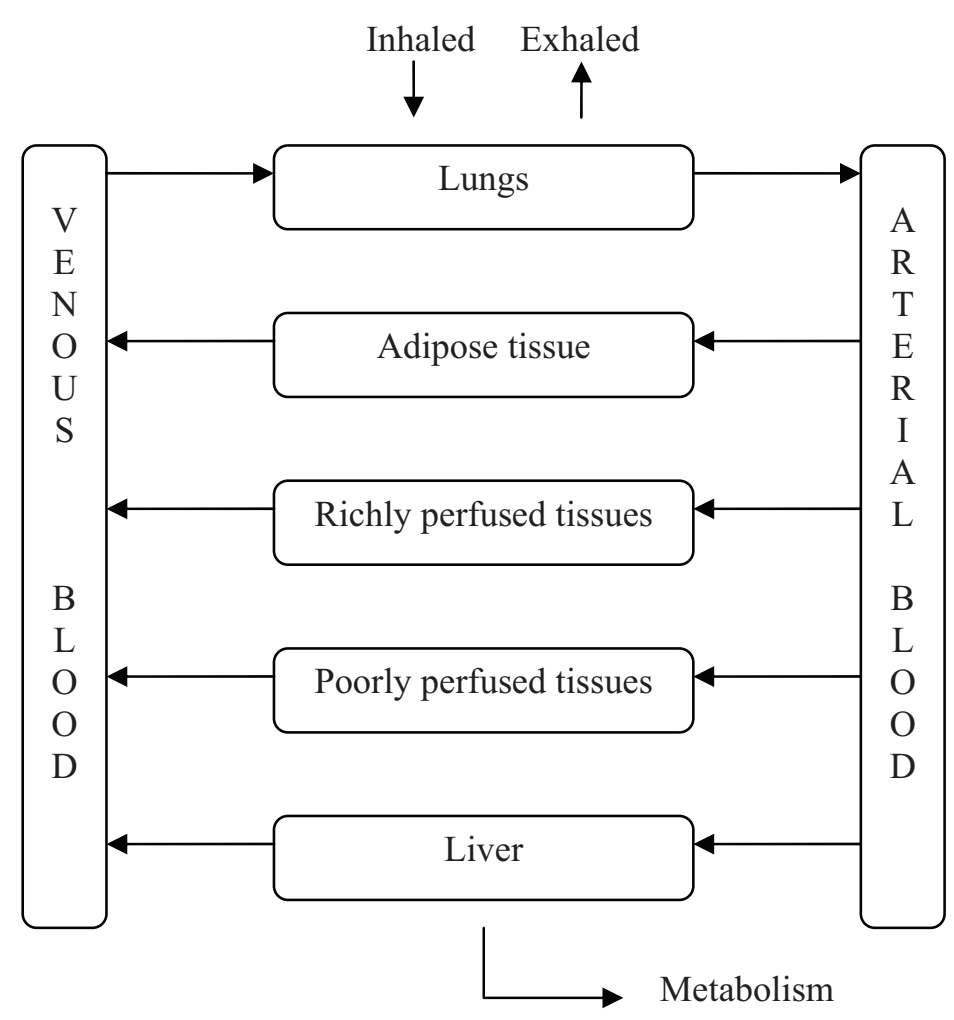

Figure 5: Illustration of the structure of an inhalation PBPK model for a volatile organic chemical.

\subsection{Mathematical description of ADME}

The equations employed in a PBPK model should be consistent with the knowledge on the mechanisms of ADME for the particular chemical (Gerlowski \& Jain, 1983; Krishnan \& Andersen, 2007). In this regard, the influx and efflux clearances of chemicals are frequently described according to the principles of perfusion limitation (i.e. compartment is considered homogeneous or well mixed; the product of permeation coefficient and surface area is much greater than the tissue blood flow rate) or diffusion limitation (i.e. compartment is heterogeneous and therefore represented as subcompartments of cellular matrix and tissue blood; the product of permeation coefficient and surface area is much less than the tissue blood flow rate) (Himmelstein \& Lutz, 1979; Gerlowski \& Jain, 1983); any departure from such principles should be documented and justified. Similarly, the type of rate equation for ADME should be consistent with biochemical evidence and first principles (e.g. Krishnan \& Andersen, 2007). A pragmatic approach is to characterize mathematical descriptions either that are different from published PBPK models or that cannot be readily and unequivocally derived from the flow diagrams.

PBPK models are based on some general assumptions regarding ADME (Rideout, 1991):

- the mixing of the chemical in the effluent blood from the tissues is instantaneous and complete;

- blood flow is unidirectional, constant and non-pulsatile; and

- the presence of chemicals in the blood does not alter the blood flow rate. 
Any deviations from such general assumptions of PBPK models should be documented, and justification should be provided.

\subsection{Computer implementation and verification}

The equations constituting the PBPK model along with the input parameters are written as a program for conducting simulations. Simulation, in the present context, refers to the system behaviour (i.e. kinetic profiles in blood and tissues) predicted by solving the differential and algebraic equations constituting the PBPK model. As PBPK models often contain differential equations (i.e. equations calculating the differential in a dependent variable, such as concentration, with respect to the independent variable, time) as well as non-linear descriptions (e.g. saturable metabolism), algorithms (e.g. fixed step-by-step procedures) are used to solve all equations simultaneously. Rideout (1991) enumerated the characteristics of acceptable algorithms in modelling. If an algorithm has previously been shown to work for PBPK models of a particular type (e.g. Euler or Runge-Kutta algorithm, in the case of volatile chemicals), then a detailed characterization of their theoretical basis or their ability to efficiently provide stable solutions is usually not needed. However, when newer or nonstandard algorithms are used, they should be characterized with respect to their mathematical basis and functioning (Rideout, 1991).

The accuracy of mathematical and computational implementations of PBPK models should be verified systematically. Model verification essentially focuses on making sure that the model is built correctly (Balci, 1997), whereas model evaluation focuses on the level of confidence in a given model for predicting the reality in the context of a specific application (section 3.7). In the context of PBPK modelling, "verification" would involve a systematic review to ensure that:

- the model codes (i.e. equations and parameter values) are devoid of syntax or mathematical errors;

- the units of input parameters and variables are accurate;

- the chemical mass balance as well as blood flow balance within the model are respected at all times; and

- there is no solver or numerical error.

The confidence in the computer implementation of a PBPK model will be increased if the model is independently coded and run in a different computer language or program.

\subsection{Parameter estimation and analysis}

The methods for estimation and analysis of chemical-specific parameters as well as biological input data for PBPK models have been reviewed in the literature (e.g. Krishnan \& Andersen, 2007; Lipscomb \& Ohanian, 2007). In the present document, emphasis is placed on characterization of the input parameters of PBPK models intended for risk assessment application, as discussed below:

- The input parameters should reflect biological or mechanistic determinants of ADME of the chemical being modelled. An example would be the fat:blood partition coefficient, which is a key determinant of the distribution and accumulation of many chemicals in adipose tissue. 
- The values (i.e. point estimates or distributions) used for physiological parameters in the model (e.g. alveolar ventilation rate, cardiac output, tissue volumes, rates of tissue blood flow, glomerular filtration rate) should be within the documented range for the particular species and life stage (e.g. ICRP, 1975; Arms \& Travis, 1988; Davies \& Morris, 1993; Brown et al., 1997; Price et al., 2003; Gentry et al., 2004; Thompson et al., 2009).

- The cardiac output specified in the model should be equal to the sum of blood flow rates to the tissue compartments.

- The ventilation:perfusion ratio should be within the physiological range for the species, age group and activity level.

- Physicochemical parameters (e.g. blood:air, tissue:plasma and tissue:blood partition coefficients) should have been obtained on the basis of independent measurements (in vitro, in vivo) or using algorithms in the valid domain of application (e.g. quantitative structure-activity relationships [QSARs], tissue composition-based algorithms) (Béliveau et al., 2005; Krishnan \& Andersen, 2007; Rodgers \& Rowland, 2007; Schmitt, 2008).

- Biochemical parameters estimated by fitting model simulations to time course data obtained under in vivo conditions should be characterized on the basis of their sensitivity to the in vivo data (Krishnan \& Andersen, 2007). If in vitro approaches are used, the validity of the in vitro system to adequately predict the kinetics of chemicals in vivo should be ensured and reported. Processes and parameters operative in vivo should be accounted for while scaling in vitro rates to the whole animal (Lipscomb et al., 1998; Barter et al., 2007; Lipscomb \& Poet, 2008).

- Allometric scaling of model parameters, if done, should be justified or explained as to the basis, appropriateness and uncertainties (Adolph, 1949; Dedrick et al., 1973; Dedrick \& Bischoff, 1980).

\subsection{Model validation and evaluation}

All models of complex real-world systems have potentially two types of built-in errors varying in magnitude and importance: 1) structural uncertainty/error and 2) parameter uncertainty/error (IPCS, 2005b). The adequacy of model structure and parameter values is often inferred by comparing model predictions with experimental data that had not been used for estimating parameters. This process is referred to as "validation", "strong validation" or "external validation" (Cobelli et al., 1984; Leijnse \& Hassanizadeh, 1994; IPCS, 2005b). Validation is defined as the process by which the reliability and relevance of a particular approach (or model, in this context) are established for a defined purpose (IPCS, 2005b). In the context of PBPK and such simulation models in risk assessment, we should really be concerned about purpose-specific "evaluation" rather than generic "validation" (Barton et al., 2007). Model evaluation is the process of establishing confidence in the model on the basis of scientific principles, the quality of the input parameters as well as the ability of the model to reproduce independent empirical data (Oreskes, 1998). It should also be noted that in the application of some statistical methods to PBPK models, notably Bayesian analysis, all available data would appropriately be used to characterize the uncertainty and variability in parameter estimates and model predictions, rather than arbitrarily excluding selected data sets for "validation" purposes (Barton et al., 2007). 
Regardless of the terminology (i.e. evaluation versus validation), the overall intent here is the same - that is, to assess the appropriateness (whether the major determinants of the system behaviour have been adequately captured by the model) and predictive ability (whether the model, with its set of input parameters, can adequately simulate the behaviour of a chemical for specific use purposes) of the model. In this regard, it is important to realize that the simulations of more than one model may agree with a limited set of data (Oreskes, 1998). Further, no model can be proven to be universally valid; that is, no model can demonstrate that it is capable of providing simulations that correspond to reality or experimental data for all relevant dose metrics in test species and humans exposed by all exposure scenarios, doses and routes of interest. Such a goal, in fact, is neither pertinent nor feasible (Cobelli et al., 1984; IPCS, 2005b). If dose metric data were available for every chemical, exposure scenario and species, then we would use the data directly and not opt for the use of PBPK models in risk assessment. The advantage of modelling is really to simulate dose metrics that were not or cannot otherwise be measured because such measurement is neither feasible nor ethical. These kinds of advantages of modelling are exemplified in some applications to epidemiological studies, where the model predicts the changes in internal exposures during the lifetime, but data are available only at a selected time (Verner et al., 2009). The issue then really becomes: what level of confidence is required for a model to be used for a specific risk assessment application, and what other alternative to the PBPK model is available for effectively addressing the TK uncertainty associated with the specific risk assessment issue/ requirement?

Section 3.7.1 discusses the characterization of the level of confidence in PBPK models, whereas sections 3.7.2 and 3.7.3 place the models in the context of their use in risk assessment.

\subsubsection{Characterizing the level of confidence in PBPK models}

The level of confidence in PBPK models intended for specific end use in risk assessment should be characterized on the basis of:

- the biological basis of the model structure and parameters;

- comparison of model simulations with experimental data; and

- reliability of model predictions of dose metrics relevant to risk assessment (model testing, uncertainty and sensitivity analyses).

\subsubsection{Biological basis}

The level of confidence in a PBPK model will be high if its structure is consistent with accepted physiological and biochemical theories. The key question here is:

\section{Do the model structure and parameters have a reasonable biological basis?}

Regardless of how well the simulations of a PBPK model match the data, its structure should not violate what is known about the physiology of the modelled organism. If the model cannot reproduce PK profiles with any realistic parameter values or it can do so only by using values that are inconsistent with the current state of knowledge, then one can reasonably conclude that the model structure or the parameters are inadequate. The following are examples of questions that relate to the biological basis of input parameters of a PBPK model: 
- Is the sum total of the tissue blood flow rates equal to the cardiac output?

- Are the tissue volumes and tissue blood flow rates within the documented range for the particular species and life stage?

- Is the ventilation:perfusion ratio specified in the model within physiological limits?

- Is the allometric scaling of parameters, if applicable, done appropriately?

Accordingly, the model assumptions, processes, parameters and structure should have a reasonable biological basis and be consistent with the available data on the PK and PD of the chemical being modelled. Thus, if biologically implausible parameters are required for the model to reproduce empirical data, then the model is wrong, the prior information about the parameters is wrong or the data are wrong. In the case of hypothetical volatile chemical VC (see Annex 3), for example, the confidence in the use of a model to perform route-to-route extrapolation is based more on the extent to which the model reflects correct physiology (e.g. the anatomical relationships of tissues and blood flows) and biochemical principles (e.g. the Michaelis-Menten equation) than on the extent of supportive empirical data. However, when the biological basis of one or more model parameters, assumptions or structural elements is questionable, the confidence in such a PBPK model will be low, thus impeding its uptake in risk assessment for making reliable extrapolations.

\subsubsection{Comparison of model simulations with data}

Confidence in a PBPK model and its parameters will be high if it reproduces a variety of data - that is, it is able to provide optimal simulation of data from more than one particular experiment using one route of administration and one dose (Gentry et al., 2004). Here the key question is:

How well does the PBPK model reproduce the chemical-specific PK data under various experimental or exposure conditions?

The comparison of PBPK models with experimental data may be done using one of several methods: visual inspection, statistical tests and discrepancy indices (Chiu et al., 2007). Statistical hypothesis tests and discrepancy tests, however, are of limited use in the context of PBPK modelling. Because the model is an approximation of the actual system, a null hypothesis that the system and the model are the same is clearly false. Plots of residuals against the fitted values, time, dose, etc. can be useful in identifying systematic errors in the model (Iyengar \& Rao, 1983).

Visual inspection, a frequently used approach, focuses on the qualitative ability of the model to reproduce the shape of the time course of chemical concentrations in biological matrices. Accordingly, if the model consistently reproduces the general trend of the data (peaks, bumps and valleys, saturation of metabolism, etc.), then there will be greater confidence in the model than if it fits only a portion of the data (e.g. absorption phase) perfectly (Chiu et al., 2007).

While attempting to compare model simulations and experimental data, it is important to remember that both are subject to uncertainty, and this should be considered in order to provide a fair comparison between the two (Marcus \& Elias, 1998; IPCS, 2008). The experimental data, frequently obtained in a few experimental animals or human subjects, may constitute a biased sample that may not reflect the entire range of values or the central tendency of the values found in reality. It must be kept in mind that all deterministic models predict mean concentrations, whereas a single experimental observation constitutes only one 
sample from the hypothetically infinite range of observations from that identical experiment (Veerkamp \& Wolff, 1996).

In PBPK modelling, predictions that are, on average, within a factor of 2 of the experimental data have frequently been considered adequate. When the training (or parameter estimation) data set and evaluation data set are obtained in different experimental animals/human subjects, as in most PBPK modelling activities, the resulting simulations are not anticipated to fit the PK data perfectly at all time points. Therefore, owing to the lack of precise knowledge (i.e. uncertainty) of parameter values for the experimental animals (or humans) used in estimating parameters versus those used for generating the PK data for model evaluation, some level of discordance is to be expected. Confidence in a PBPK model will be greater if it adequately simulates the PK data that were used during calibration as well as those that were not part of the calibration data set. However, the value of the model should not be judged on the basis of closeness to data alone (Rescigno \& Beck, 1987); consideration of biological basis (section 3.7.1.1) and reliability of dose metric predictions (section 3.7.1.3) should be an integral part of this process.

\subsubsection{Reliability of dose metric predictions (model testing, uncertainty and sensitivity analyses)}

The level of confidence in a PBPK model intended for use in risk assessment depends critically on its ability to provide reliable predictions of dose metrics. It is therefore important to evaluate whether the model is trustworthy with respect to its predictions of the dose metric for the risk assessment. Here the key question is:

How reliable is the PBPK model with regard to its predictions of dose metrics relevant to risk assessment?

The degree of confidence in the predictions of dose metrics by a PBPK model depends upon how well it has been tested against real data and whether adequate sensitivity and uncertainty analyses have been conducted to support the reliability of the predictions. Whereas the uncertainty analysis evaluates the impact of the lack of precise knowledge of parameter values and model structure on dose metric simulations (Figure 6), the sensitivity analysis provides a quantitative evaluation of how input parameters influence the dose metrics or other model output of relevance to the risk assessment (Figure 7) (Iman \& Helton, 1988; Farrar et al., 1989; Krewski et al., 1995; Campolongo \& Saltelli, 1997; Nestorov, 2001; Gueorguieva et al., 2006b; Chiu et al., 2007; Loizou et al., 2008). If the sensitivity analysis indicates that a small change in a parameter value (i.e. less than typical variability in its measurement) would lead to changes in predictions of a dose metric that are less than the variation expected from its experimental measurement, then the model is considered to be reliable. Conversely, if the dose metrics simulated by a model are not sensitive to large variations or errors in expected input parameters, then the predictions would be considered of questionable reliability for risk assessment applications (Kohn, 1995). Figures 8 and 9 illustrate the outcome of sensitivity and uncertainty analyses of PBPK models for the hypothetical chemical VC (see Annex 3). These figures provide a convenient template for considering the relative reliability of PBPK models on the basis of uncertainty and sensitivity. 


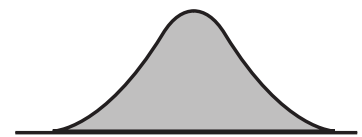

Physlologlcal

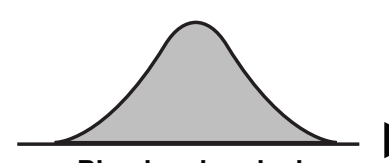

Physlcochemical

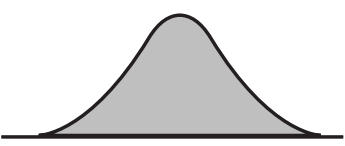

Blochemical

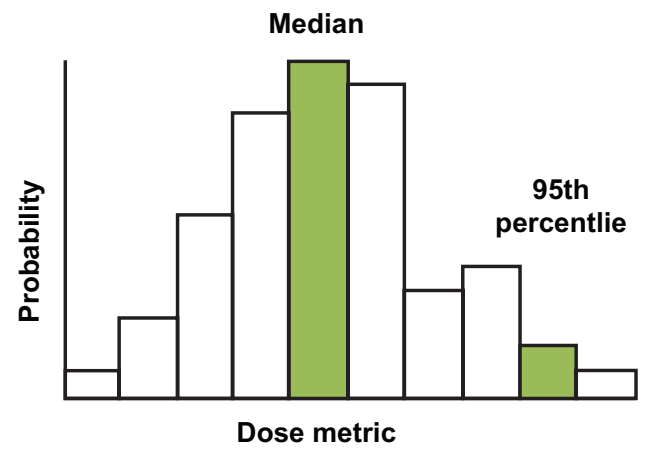

Dose metric

Input parameter distributions

Figure 6: An illustration of uncertainty analysis in PBPK models. The ratio of the 95th percentile value over the median value of the dose metric is a measure of the uncertainty in dose metric predictions.

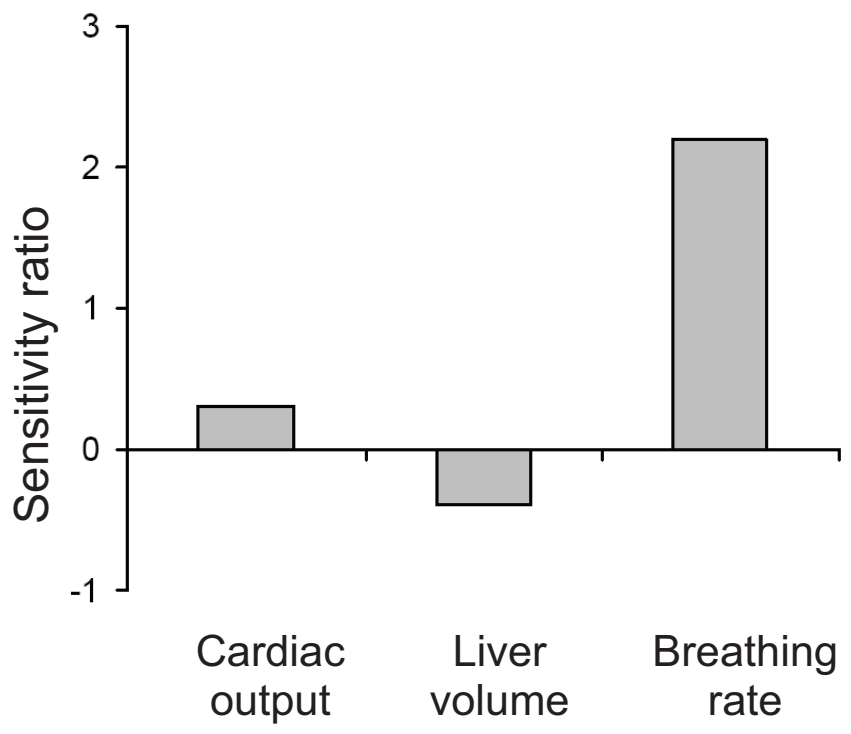

Figure 7: Illustration of the output of sensitivity analysis of PBPK models. A sensitivity ratio of 1 implies that a $1 \%$ change in parameter value (i.e. input) leads to a $1 \%$ change in output (i.e. dose metric predictions) of the model, indicating that the output is sensitive to that input parameter under the conditions evaluated. 


\begin{tabular}{|c|c|c|c|c|}
\hline & & \multicolumn{3}{|c|}{ UNCERTAINTY } \\
\hline & & High & Medium & Low \\
\hline \multirow{3}{*}{ 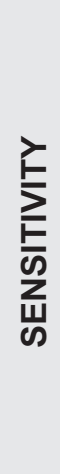 } & 동 & 一 & Cardiac output & $\begin{array}{l}\text { Liver volume } \\
\text { Blood:air partition coefficient } \\
\text { Liver blood flow }\end{array}$ \\
\hline & $\begin{array}{l}\frac{\mathrm{g}}{\mathrm{J}} \\
\frac{0}{0} \\
\sum^{\frac{0}{2}}\end{array}$ & 一 & Pulmonary ventilation & Body weight \\
\hline & วิ & - & - & $\begin{array}{c}V_{\max } \\
K_{\mathrm{m}}\end{array}$ \\
\hline
\end{tabular}

Figure 8: The output of the uncertainty and sensitivity analyses for the rat VC PBPK model (exposure conditions: $128 \mathrm{mg} / \mathrm{m}^{3}, 4 \mathrm{~h} /$ day, inhalation). Sensitivity analysis results are presented as high (absolute value greater than or equal to 0.5), medium (absolute value greater than or equal to 0.2 but less than 0.5 ) or low (absolute value greater than or equal to 0.1 but less than 0.2 ); parameters with sensitivities less than 0.1 are not listed. Uncertainty analysis results are summarized as high uncertainty (value could be a factor of 2 or higher), medium uncertainty (value could be a factor between 0.3 and 2) or low uncertainty (value could be a factor of 0.3 or lower). As per Figure 6 , uncertainty is defined as the ratio of the 95th percentile over the median value for the dose metric. As per Figure 7, a sensitivity ratio of 1 implies that a $1 \%$ change in input of a parameter value leads to a $1 \%$ change in dose metric prediction. $K_{\mathrm{m}}$, Michaelis-Menten constant; $V_{\max }$, maximal rate of metabolism.

\begin{tabular}{|c|c|c|c|c|}
\hline & & \multicolumn{3}{|c|}{ UNCERTAINTY } \\
\hline & & High & Medium & Low \\
\hline \multirow{3}{*}{ 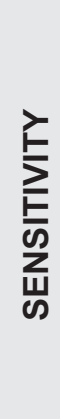 } & 告 & - & - & $\begin{array}{l}\text { Liver volume } \\
\text { Blood:air partition coefficient }\end{array}$ \\
\hline & $\begin{array}{l}\frac{\xi}{D} \\
\frac{\bar{z}}{0} \\
\sum\end{array}$ & - & $\begin{array}{c}\text { Cardiac output } \\
V_{\max } \\
K_{\mathrm{m}}\end{array}$ & Liver blood flow \\
\hline & 3 & - & Pulmonary ventilation & Body weight \\
\hline
\end{tabular}

Figure 9: The output of the uncertainty and sensitivity analyses for the human VC PBPK model (exposure conditions: $2.6 \mathrm{mg} / \mathrm{m}^{3}$, continuous inhalation exposure). Sensitivity analysis results are presented as high (absolute value greater than or equal to 0.5 ), medium (absolute value greater than or equal to 0.2 but less than 0.5 ) or low (absolute value greater than or equal to 0.1 but less than 0.2 ; parameters with sensitivities less than 0.1 are not listed. Uncertainty analysis results are summarized as high uncertainty (value could be a factor of 2 or higher), medium uncertainty (value could be a factor between 0.3 and 2) or low uncertainty (value could be a factor of 0.3 or lower). As per Figure 6, uncertainty is defined as the ratio of the 95th percentile over the median value for the dose metric. As per Figure 7, a sensitivity ratio of 1 implies that a $1 \%$ change in input of a parameter value leads to a $1 \%$ change in dose metric prediction. 
The benefit of uncertainty and sensitivity analyses might be limited for chemicals such as $\mathrm{VC}$, for which the PBPK model was evaluated using extensive data sets representing wide dose ranges, exposure routes and species (see Annex 3). Conversely, the uncertainty analysis is likely to be useful when a PBPK model does not adequately simulate the experimental data or when there has been only very limited evaluation of its ability to simulate the dose metrics. For example, in the case of vinyl acetate (Bogdanffy et al., 1999, 2001), the simulations of dose metric (i.e. change in intracellular $\mathrm{pH}$ ) by the PBPK model could not be evaluated with experimental data in laboratory animals or humans for the exposure scenarios of interest to risk assessors (a technical feasibility issue). In such cases, only some other aspects of the model could be evaluated (e.g. nasal outflow), such that uncertainty and sensitivity analyses would help address concerns of model reliability (Hattis et al., 1993; Clewell et al., 1994).

Development and application of PBPK models in risk assessment frequently involve three kinds of situations for which sensitivity and uncertainty analyses are valuable. These conditions are applicable to 1) human risk assessment, 2) experimental animal or human studies from which toxicity end-points are obtained and 3) TK studies available to support the model. It is critical to examine, for the human risk assessment conditions (e.g. exposure pathways, relevant exposure conditions such as acute or chronic), the model parameters upon which the dose metric predictions depend (i.e. those to which they are sensitive). It is also important to identify the parameters upon which dose metric predictions depend through a sensitivity analysis for the experiments from which end-points are derived (i.e. toxicity, epidemiological or clinical studies). Then, it is useful to assess whether the uncertainty in those parameters could be characterized either qualitatively or quantitatively-for example, by demonstrating that the available PK or tissue dosimetry data determine well parameter values important for the prediction of the relevant dose metric in the preceding two situations. This would depend to some extent on whether the dose metric is measurable (e.g. plasma concentrations of a stable compound) or has to be inferred (e.g. reactive metabolite, concentration of free compound in the brain). For example, the use of parent chemical data to test the model estimates of metabolism in the organism can, in some cases, be misleading, because the metabolism parameters may have little impact on bulk parent chemical concentrations. In this case, the level of confidence associated with a PBPK model may be improved by time-dependent sensitivity analysis to verify whether, in fact, the metabolic parameter values are identifiable from the available data sets. Ideally, a PBPK model should be compared with data that are informative regarding the parameters to which the dose metric predictions are sensitive. This presupposes the use of sensitivity and uncertainty analyses to identify the parameters of concern (i.e. those that are least certain but have the most influence on the dose metric). The case-study template in Annex 3 recommends, then, characterizing the parameters to which risk assessment predictions are sensitive in section A2.3, while appending, wherever possible, a table of all the parameter values. Table B1.1 of Annex 3 presents the numerical values of key parameters of the PBPK models for chemical VC - that is, those parameters that are most influential with regard to the dose metric predictions of relevance to the risk assessment of chemical VC.

In situations where the extrapolation uncertainty is low-that is, when the PBPK model has been evaluated using dose metric data collected for the species, life stage, exposure route, dose range (linear versus saturable doses) and activity levels (physical activity versus resting condition) of relevance to the risk assessment - the use of the PBPK model would be essentially to make limited extrapolations (e.g. to somewhat lower doses or a different physical activity level). For example, in the case of 2-butoxyethanol (Johanson \& Johnsson, 1991; Corley et al., 1994; Lee et al., 1998; Environment Canada \& Health Canada, 2002), the 
AUC for the metabolite was reported only for the post-exposure period in the critical toxicological study. Therefore, the PBPK model was used to calculate the total AUC (i.e. during exposure + post-exposure) in test animals for the purpose of computing the $\mathrm{AK}_{\mathrm{AF}}$. Further, for this chemical, the published data on AUC in humans were collected under exercising conditions, and these might not be reflective of the kinetics under resting conditions. Here, the PBPK model facilitated the estimation of dose metric (i.e. the AUC during resting conditions) on the basis of appropriate physiological parameters. Therefore, the reliability of the use of a PBPK model for conducting limited extrapolation - in this case, to predict the relevant dose metrics of 2-butoxyethanol in rats and humans-would be considered to be high (Table 2).

\section{Table 2: Application for interspecies extrapolation: characterizing the level of confidence in a PBPK model-2-butoxyethanol example.}

\begin{tabular}{ll}
\hline Model evaluation aspect & Observation \\
\hline Biological basis & $\begin{array}{l}\text { The model parameters, structure and assumptions have reasonable } \\
\text { biological basis and are consistent with available data. }\end{array}$ \\
Model simulations of data & $\begin{array}{l}\text { The rat and human PBPK models, with a single set of species- } \\
\text { specific parameters, simulated the PK profile (i.e. bumps and } \\
\text { valleys) of 2-butoxyethanol and its metabolites following inhalation } \\
\text { exposure (the route of relevance to the risk assessment) in rats and } \\
\text { humans. }\end{array}$ \\
$\begin{array}{l}\text { Reliability (model testing, } \\
\text { uncertainty and sensitivity) }\end{array}$ & $\begin{array}{l}\text { The model simulations were compared with measured data on the } \\
\text { toxic moiety reflective of the dose metric (i.e. blood concentrations of } \\
\text { metabolites) 1) in rats exposed by inhalation to atmospheric } \\
\text { concentrations in the range of the dose-response study (150-1210 } \\
\text { mg/m³) as well as 2) in humans exposed to a low concentration (i.e. } \\
\text { first-order range) of relevance to the risk assessment. }\end{array}$ \\
\hline
\end{tabular}

When the dose metric is technically not measurable by currently available methods or is less well determined by the available calibration methods, the reliability of the PBPK model to generate dose metric predictions should be assessed on the basis of focused sensitivity and uncertainty analyses. Figure 10 illustrates how the outcome of sensitivity and uncertainty analyses might inform the reliability of a model to provide dose metric predictions of use in risk assessment. An example might be a chemical for which metabolite concentrations in specific cell types in nose or lung are relevant but are technically not measurable with currently available methods or are poorly determined by in vivo calibration data (e.g. because they represent a very small fraction of the overall PK). This situation is illustrated with vinyl acetate, for which the toxicity and carcinogenicity are hypothesized to be associated with a reduction in intracellular pH caused by one of its metabolites (Bogdanffy et al., 1999, 2001). As it is not possible to make measurements of the time course of $\mathrm{pH}$ changes in nasal tissues of exposed experimental animals and humans, the PBPK model was used for making predictions. Confidence in this PBPK model was established by comparing simulations with data on nasal flow rates, deposition in upper airways as well as metabolite production and release via the nasal cavity for various exposure concentrations in the rat. The model also consistently simulated data on concentrations of vinyl acetate and its metabolite in the airstream of the nasopharyngeal cavity during controlled human exposures to low concentrations $\left(3.5-35 \mathrm{mg} / \mathrm{m}^{3}\right)$. Thus, the level of confidence in this model for the intended purpose was characterized as medium, based on the considerations of biological basis, performance and reliability (the latter being supported by the results of sensitivity and uncertainty analyses) (Table 3 ). 


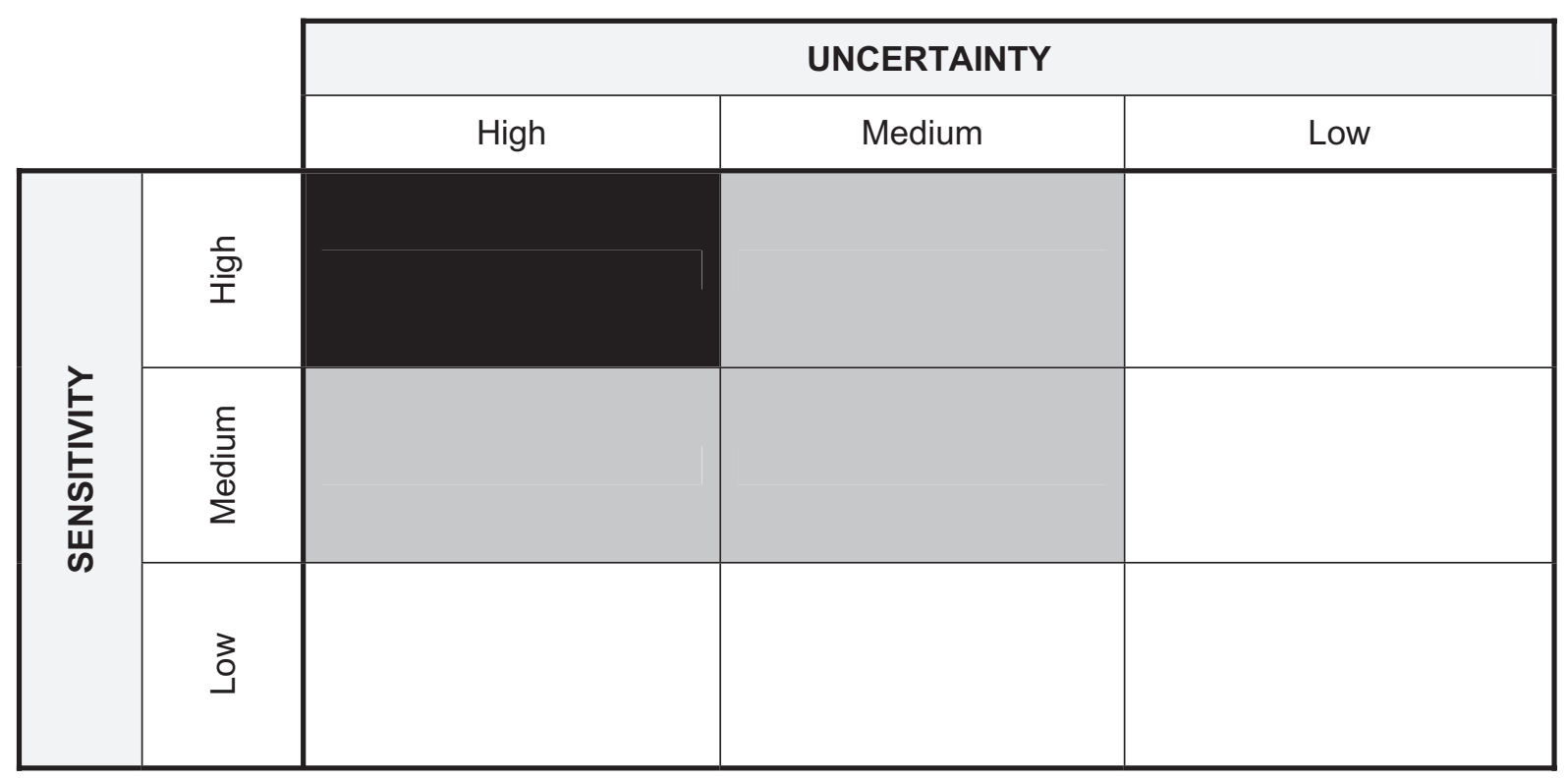

Figure 10: Illustration of the role of sensitivity and uncertainty analyses in determining the reliability of PBPK model predictions of dose metrics for risk assessment. High reliability ( $\square$ ); Medium reliability $(\square)$; Low reliability $(\square)$.

\section{Table 3: Application for interspecies extrapolation: characterizing the level of confidence in a PBPK model-vinyl acetate example.}

\begin{tabular}{|c|c|}
\hline Model evaluation aspect & Observation \\
\hline Biological basis & $\begin{array}{l}\text { The five-compartment PBPK model of the rat nasal cavity and four- } \\
\text { compartment human nose model are consistent with current } \\
\text { knowledge of upper airways; the model parameters and } \\
\text { assumptions have reasonable biological basis. }\end{array}$ \\
\hline Model simulations of data & $\begin{array}{l}\text { The PBPK model was applied to simulate in vivo experiments } \\
\text { designed to measure uptake and metabolism of vinyl acetate in the } \\
\text { upper respiratory tract of rats exposed to atmospheric } \\
\text { concentrations ranging from } 260 \text { to } 7710 \mathrm{mg} / \mathrm{m}^{3} \text { for } 1 \mathrm{~h} \text {. Also, the } \\
\text { experimental data on the extraction of vinyl acetate from air and } \\
\text { release of metabolites into the airstream were simulated by the rat } \\
\text { PBPK model. Overall, predictions of the vinyl acetate PBPK model } \\
\text { were compared against limited data sets that included multiple } \\
\text { concentrations, nasal flow rates, deposition in upper airways and } \\
\text { metabolite production and release via the nasal cavity. }\end{array}$ \\
\hline $\begin{array}{l}\text { Reliability (model testing, } \\
\text { uncertainty and sensitivity) }\end{array}$ & $\begin{array}{l}\text { The model predictions of dose metrics have not been compared } \\
\text { against directly relevant data (e.g. pH changes in nasal tissues of } \\
\text { rats or humans); however, the model reproduced the PK of vinyl } \\
\text { acetate and its metabolites in the upper respiratory tract of rats. The } \\
\text { human PBPK model was not evaluated with empirical data, but } \\
\text { sensitivity analyses were conducted to identify key parameters } \\
\text { determining the dose metric in rats and humans. }\end{array}$ \\
\hline
\end{tabular}

In summary, comparison of simulations with available PK data is not the only basis for developing confidence in a PBPK model for application in risk assessment; equally important are aspects relating to the biological basis and reliability of dose metric predictions supported by variability, uncertainty and sensitivity analyses. 


\subsubsection{Ability of PBPK models to address PK uncertainty relative to other approaches}

The ability of a PBPK model, relative to the other available approaches, for reducing the sources of PK uncertainty in risk assessment can be assessed on the basis of the 1) choice of dose metrics, 2) conceptual model and 3) input parameters.

\subsubsection{Dose metric}

The dose metric related to a toxicological end-point may be the maximal concentration, the AUC or the daily average concentration or amount of the parent chemical, metabolites or a combination thereof. Dose metrics may also be expressed in relation to free or total concentrations (Kalvass \& Maurer, 2002). The use of a PBPK model allows one to evaluate these potential dose metrics on the basis of the current state of knowledge on the MOA of the chemical and to choose the appropriate dose metric for conducting the risk assessment. In such cases, then, there is greater certainty than for default approaches in the dose metric used as the basis for conducting extrapolations in risk assessment, as it is supported by the MOA information and toxicological database as well as the dose-response analysis using the model. In contrast, the default approach based on "applied dose" does not make use of relevant data (whether available or not) to inform the dose metric for an assessment and does not address the uncertainty related to the choice of dose metric used in risk assessment (e.g. applied dose). Of course, when there is no information on the MOA of a chemical or when the PBPK model is not capable of providing estimates of candidate dose metrics, the PBPK analysis cannot be of use in addressing or reducing the uncertainty any more than the default approach.

\subsubsection{Conceptual model}

When a conceptual model based on nonspecific empirical observations (e.g. body surface scaling) is used in risk assessment, the level of uncertainty associated with it might be high, given that it does not take into account chemical-specific information on dose metrics and MOA. In the absence of any useful information regarding the appropriate dose metrics or potential toxic moiety, it would not be clear as to the benefit of choosing one approach over another (e.g. PBPK over default or vice versa). Even in such cases, an estimate of the absorbed dose or the circulating concentration of parent chemical may be obtained confidently with the PBPK models for specific use purposes. The confidence in the use of PBPK models over the default approaches then arises from the biological basis of these models and their ability to simulate dose metrics of relevance to risk assessment for addressing and characterizing the sources of PK uncertainty.

For some chemicals, a number of plausible default approaches are available, and none is chemical specific or relevant based on MOA. For example, in the case of the chemical vinyl acetate, three default approaches are available for the conduct of interspecies extrapolation: 1) consideration of species independence and equivalence of air concentrations; 2) dose adjustment based on amount absorbed per body mass; and 3) species differences in respiratory volume and surface area of extrathoracic region. None of these default approaches to extrapolate exposures from experimental animals to humans takes into account the metabolism in the target tissue or the concentration of the toxic moiety in the target tissue, as facilitated by a PBPK model. In this case, then, the biological basis of the PBPK model makes it a better tool for addressing PK uncertainty in interspecies extrapolation, compared with the default approaches. 


\subsubsection{Input parameters}

The numerical values of input parameters used in some default approaches (e.g. body surface scaling) are not specific to the chemicals, and this is only one of several options for the interspecies PK extrapolation of a given chemical (Figure 11). In fact, the hepatic clearance for a given chemical might vary from 0 to about $100 \mathrm{l} / \mathrm{h}$ in humans. Similarly, in experimental animals, the hepatic clearance can vary between 0 and about $1.25 \mathrm{l} / \mathrm{h}$ (based on hepatic blood flow rate). Hypothetically, then, the plausible human to experimental animal ratios of clearance for a chemical can range from infinitesimally small values to infinitely large values. This enormous uncertainty associated with generic or default approaches can be reduced or addressed by using chemical-specific information on the rate and affinity of metabolism along with other key PK determinants in PBPK models. As seen with the hypothetical chemical VC (see Annex 3), the use of the PBPK model facilitates a better understanding of the plausible value (or the range) of the clearance ratios as well as the determinants of the dose metric, thus reducing the extent of uncertainty (i.e. the range of theoretically possible answers) compared with the default approaches, which do not account for chemical-specific or species-specific data on PK determinants.

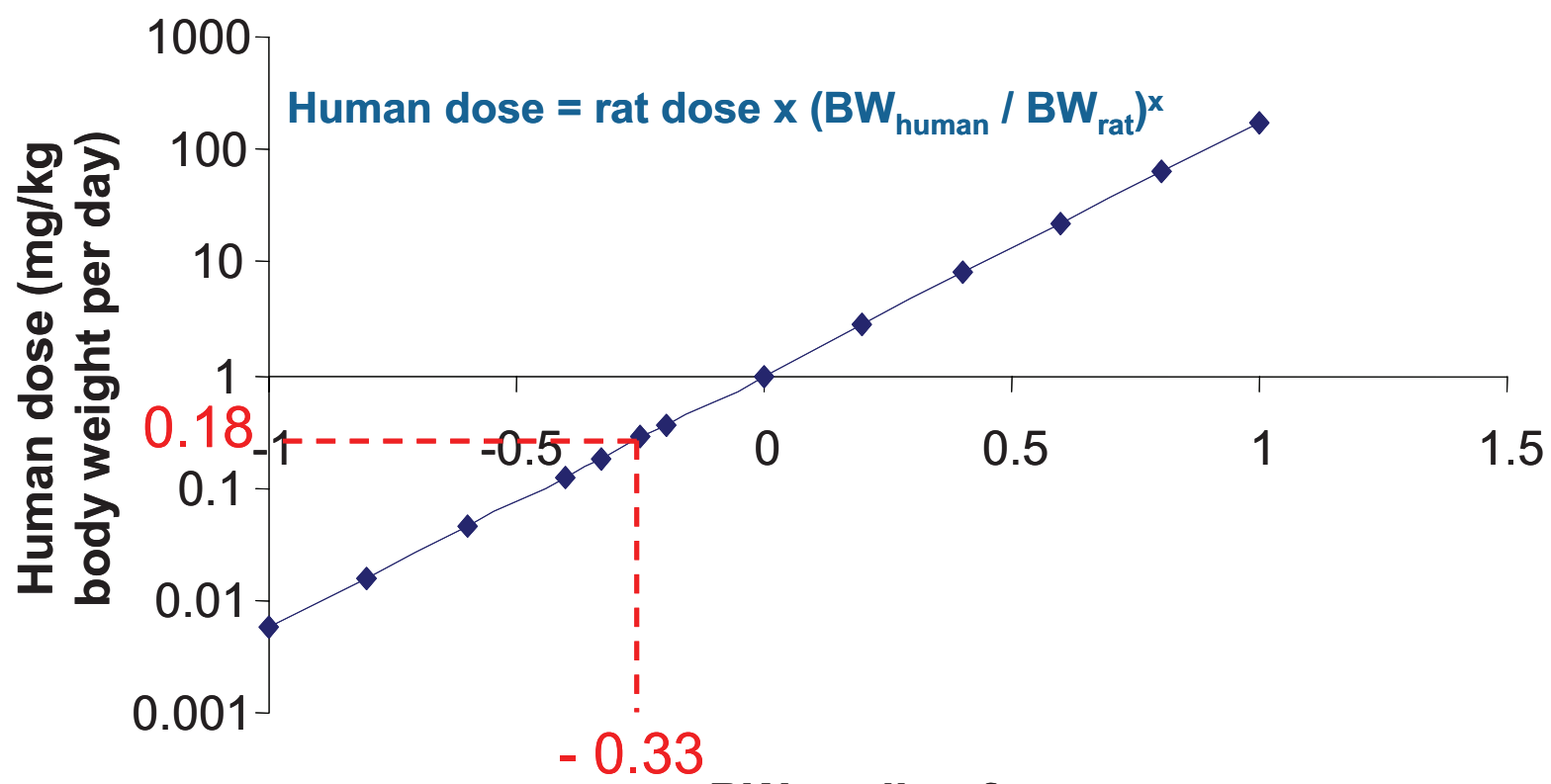

BW scaling factor

Figure 11: Illustration of the impact of the numerical values of a key input parameter of the default approaches (i.e. body weight-based scaling factor; $\left(\mathrm{BW}_{\text {human }} / \mathrm{BW}_{\text {rat }}{ }^{\mathrm{x}}\right)$ ) on the outcome (i.e. human dose estimated from rat dose, in $\mathrm{mg} / \mathrm{kg}$ body weight per day). The body surface scaling approach (i.e. (BW human $\left./ \mathrm{BW}_{\text {rat }}\right)^{-0.33}$; represented by the dotted lines) is only one of the possible options for the interspecies PK extrapolation of a given chemical.

\subsubsection{Purpose-specific model evaluation}

The following sections (sections 3.7.3.1-3.7.3.4) examine the model evaluation principles for some specific applications in risk assessment: interspecies extrapolation, interindividual variability, high dose to low dose extrapolation and route-to-route extrapolation. 


\subsubsection{Interspecies extrapolation}

For interspecies extrapolation or in the context of $\mathrm{AK}_{\mathrm{AF}}$, the PBPK model is used to calculate how the dose metric compares between an experimental animal and a human. The confidence in a PBPK model intended for use in cross-species extrapolation to humans would be high if 1) its structure and parameters have reasonable biological basis, 2) the model has been tested against PK data in different species, including humans, and 3) the reliability of the model predictions of dose metrics has been established (Figure 12). If no PK data are available in humans or if only parent chemical data are available in humans (and not the relevant dose metric, i.e. concentration of metabolite), then the reliability of the model predictions of dose metrics should be evaluated on the basis of appropriate sensitivity and uncertainty analyses. In such cases, however, the correspondence of metabolite predictions with data in several experimental animal species could be used as a surrogate, but this deficiency should be carefully considered when applying the model to predict human metabolism, as was done with chemical VC (see Annex 3).

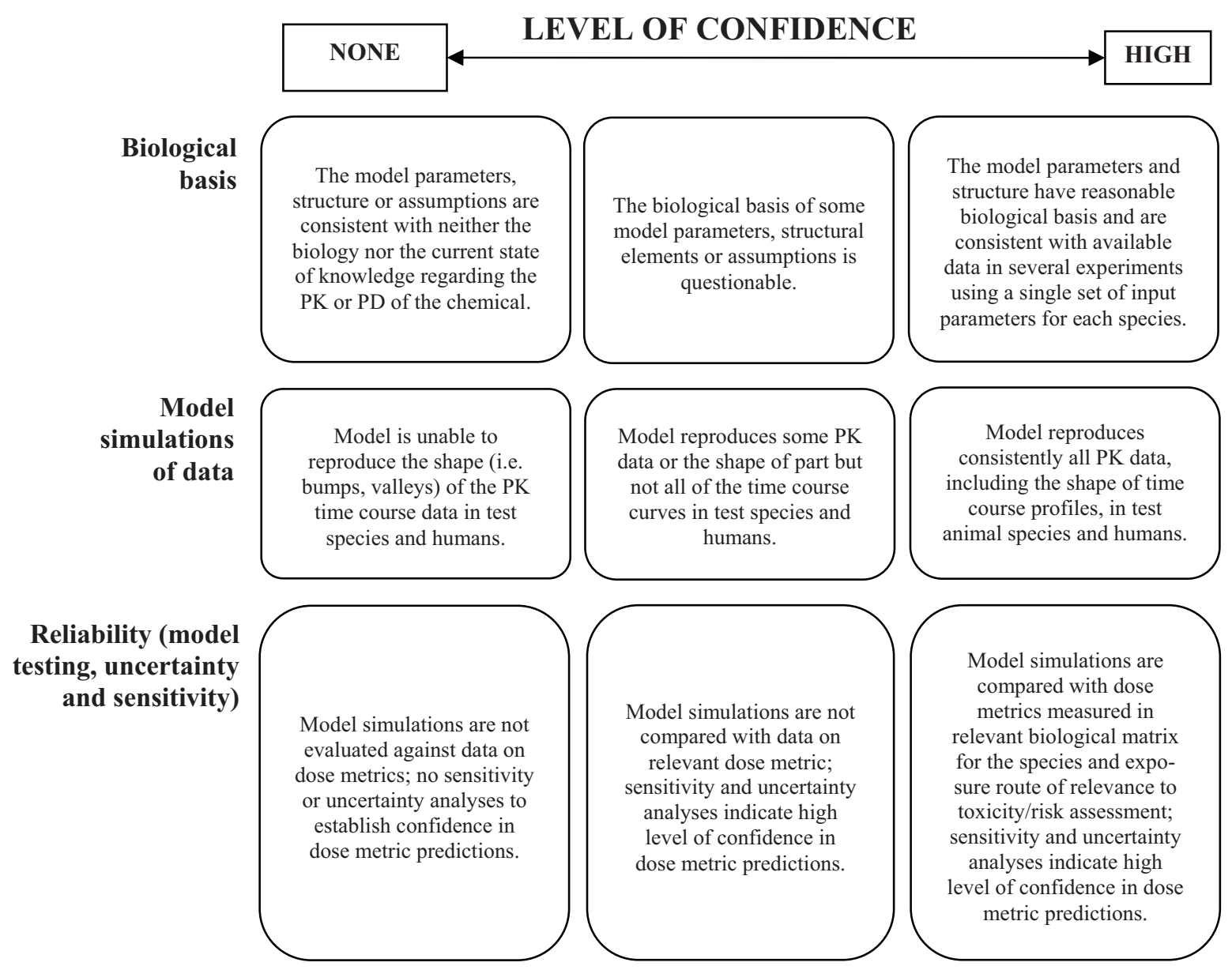

Figure 12: An illustrative scale of confidence level in PBPK models intended for use in interspecies dose extrapolation based on central tendencies. 


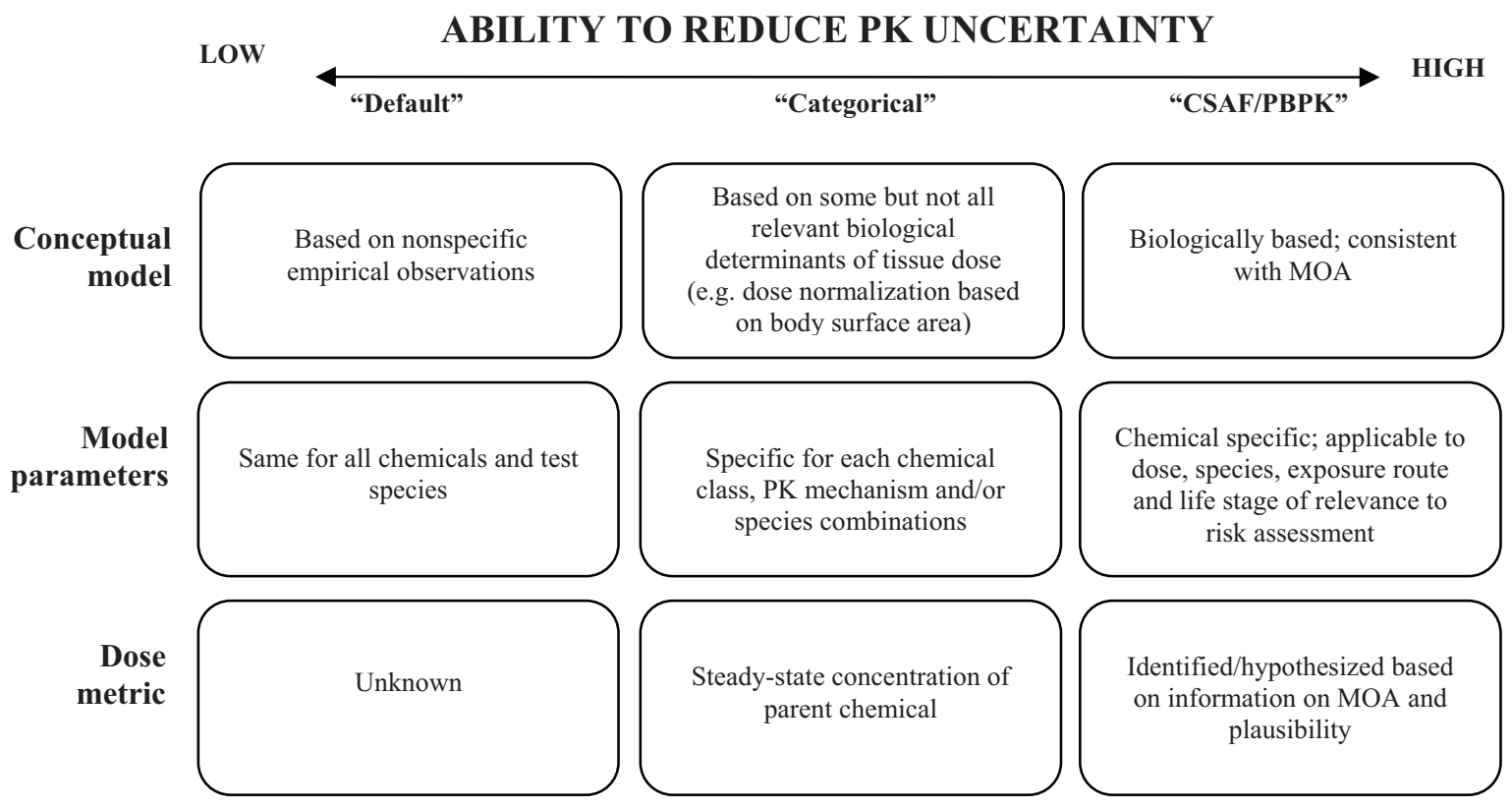

Figure 13: Relative ability of increasingly data-informed approaches in reducing the PK uncertainty associated with interspecies extrapolation.

Given that the interspecies extrapolation aspect focuses on the evaluation of the central tendency (i.e. the ratio of median values of the kinetic parameters between test animals and humans), simple PBPK models that have not incorporated information on the distribution of input parameters have been used for this purpose (USEPA, 2006). Regarding the uncertainty in the "average" values of parameters of a PBPK model used for interspecies extrapolation, a relevant question is:

What is the impact of this parameter uncertainty on the simulations of dose metrics relative to the uncertainty associated with the use of the available alternative approach (i.e. the default)?

The uncertainty related to the available alternative (i.e. default) approach arises from the conceptual model (based on nonspecific empirical observations), parameters (e.g. same for all chemicals and species; often based on average body weights of, for example, $0.25-0.40 \mathrm{~kg}$ for rats and $60-70 \mathrm{~kg}$ for humans) as well as the toxic moiety (i.e. unknown) (Figure 13). In this regard, PBPK models offer an opportunity to incorporate more data (i.e. chemicalspecific dose metrics estimated on the basis of physiological, biochemical and physicochemical determinants in both test species and humans), relative to the default approaches, to inform the adequacy of or reduce the uncertainty associated with dose extrapolation across species. In the case of 2-butoxyethanol, for example (Environment Canada \& Health Canada, 2002; USEPA, 2010), the overall confidence in the use of a PBPK model for interspecies extrapolation was high based on considerations of biological basis, model performance and reliability of dose metric predictions (see Table 2 above).

In the case of vinyl acetate, contrary to the situation with 2-butoxyethanol, the PBPK model predictions of the relevant dose metric (i.e. $\mathrm{pH}$ changes in nasal tissues of rats or humans) were not tested against empirical data (see Table 3 above). However, the model adequately simulated PK in the upper respiratory tract in both rats and humans, and a sensitivity analysis of the dose metric of relevance to risk assessment was conducted to provide additional 
support (Bogdanffy et al., 1999, 2001). Therefore, the level of confidence in the PBPK model for the conduct of interspecies PK extrapolation of vinyl acetate was considered to be medium.

\subsubsection{Interindividual variability}

For assessing human variability, a PBPK model might be initially evaluated with PK data from several individuals or a population subgroup and subsequently expanded to include estimates of distributions of input parameters. Evaluation of the human PBPK model based on comparison with data on dose metrics in the whole population is precluded. Rather, the goal would be to obtain an estimate of the distribution of key (or sensitive) parameters at the population level and incorporate them within PBPK models to characterize dose metric distributions (Figure 14). Quantifying and separating mean uncertainty from true, biologically based interindividual variability by using hierarchical and model parameter structures can be very useful when sufficient data are available (Barton et al., 2007). Several investigators would argue that instead of using any human data for model evaluation, all available human data should be used to improve the parameter estimates, so that no data are "wasted" towards that end. Such an iterative approach to model evaluation and calibration maximizes the use of the available human data. In this regard, Bayesian analysis utilizing Markov chain Monte Carlo simulation is being increasingly implemented in PBPK models to refine parameter values on the basis of information contained in additional data (e.g. Gelman et al., 1996; Bernillon \& Bois, 2000; Bois, 2000a,b; Jonsson \& Johanson, 2001, 2002; Gueorguieva et al., 2006a). However, it is necessary to evaluate whether the resulting parameter distributions adequately characterize variability in the whole population and, when they do not, utilize other data and approaches (e.g. Monte Carlo simulations) to estimate population variability.

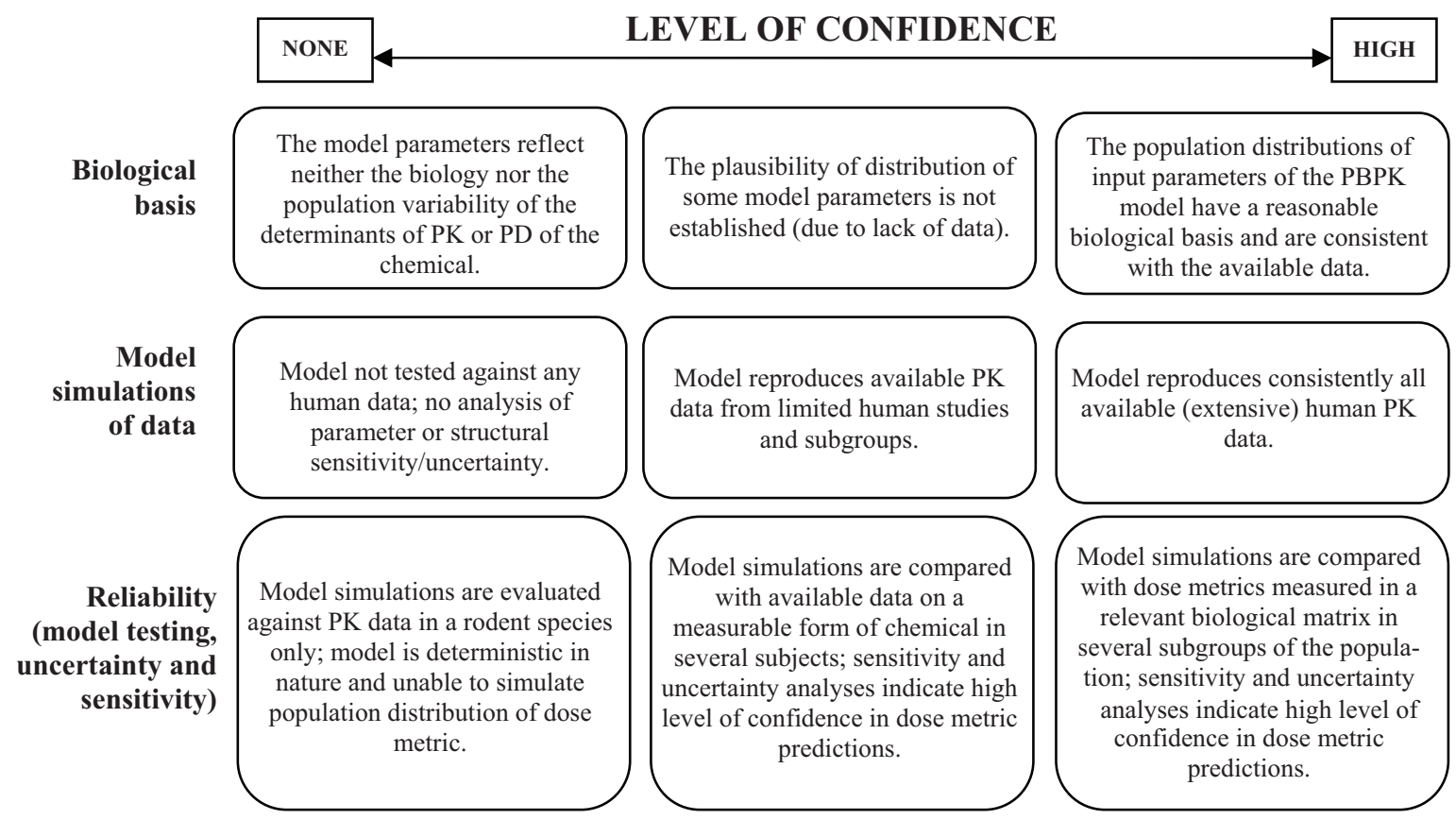

Figure 14: An illustrative scale of confidence level in PBPK models intended for evaluation of human variability in dose metrics. 


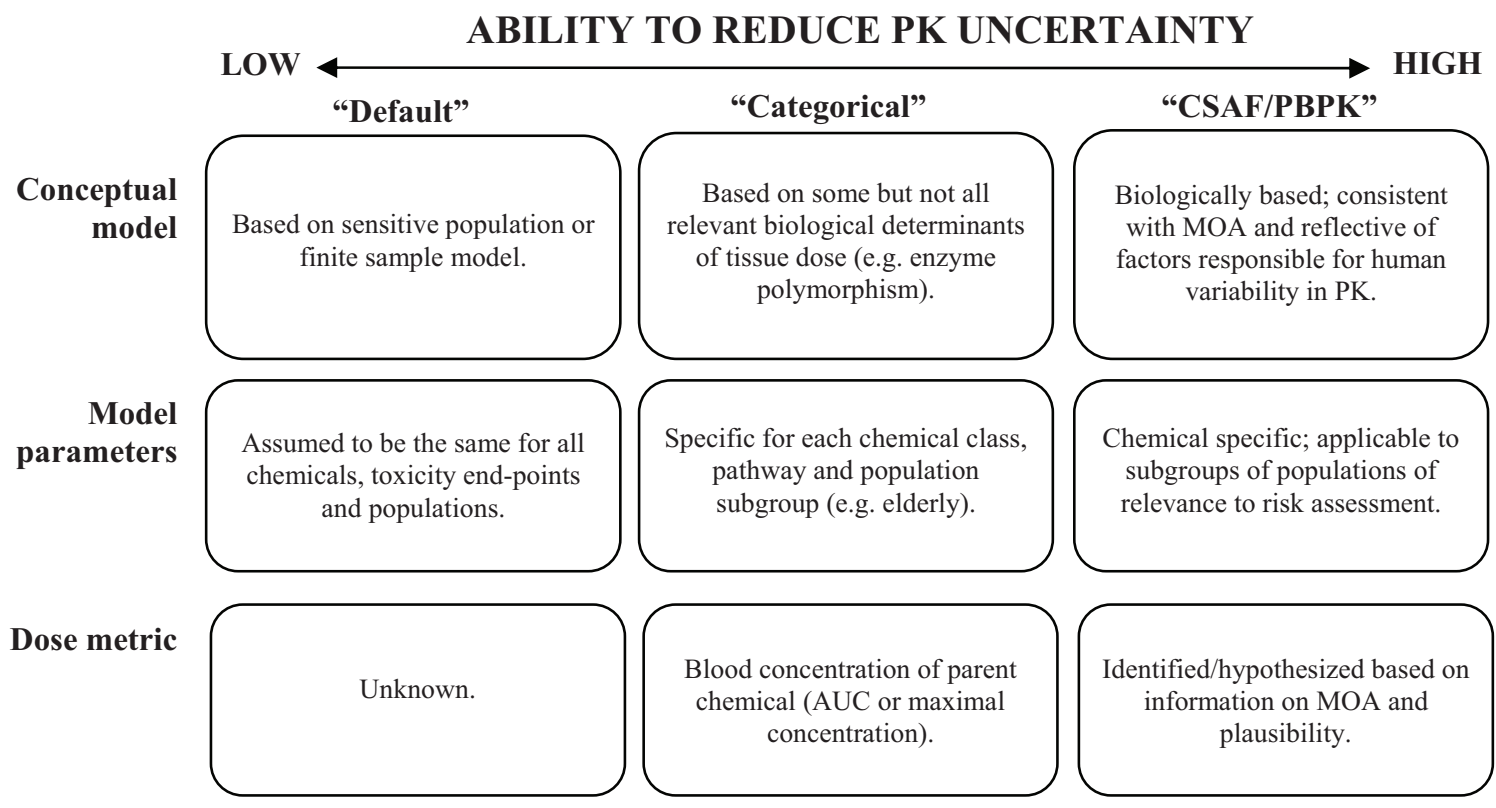

Figure 15: Relative ability of increasingly data-informed approaches in reducing the PK uncertainty associated with human variability in risk assessment.

Again, this issue should be viewed relative to the ability of the available alternatives (i.e. default approaches) to address the uncertainty associated with the characterization of individual variability in a human population or $\mathrm{HK}_{\mathrm{UF}}$. The uncertainty related to the default $\mathrm{HK}_{\mathrm{UF}}$ arises from the conceptual models (i.e. sensitive population model, finite sample model) (Price et al., 1999) and lack of consideration of a toxic moiety as well as other chemical-specific or population-specific determinants. The PBPK models offer an opportunity to incorporate relevant data to inform the adequacy of (or reduce the uncertainty associated with) use of an $\mathrm{HK}_{\mathrm{UF}}$ of 3.16 to account for the difference in dose metric between the median and sensitive individuals in a population; specifically, they facilitate the simulation of dose metrics of relevance to MOA on the basis of heterogeneity of physiological, biochemical and physicochemical determinants in human populations (e.g. Haber et al., 2002). In this regard, the effect of variability of sensitive parameters in the population would be characterized for the influence of these parameters on the dose metric when the PBPK model is intended for estimating chemical-specific interindividual variability or the CSAF for human variability in TK ( $\left.\mathrm{HK}_{\mathrm{AF}}\right)$ (e.g. Allen et al., 1996). For example, data on enzyme polymorphism and distributions of physiological parameters have been integrated within a human PBPK model for parathion to facilitate the characterization of the population distribution of dose metrics (Haber et al., 2002). If one were using the alternative (default) approaches, then these highly relevant data could not otherwise be used judiciously for characterizing human variability (Figure 15).

\subsubsection{High dose to low dose extrapolation}

For the purpose of high dose to low dose extrapolation, the evaluation of PBPK model performance should be undertaken in the dose range of relevance (e.g. linear and saturable dose ranges). Here, the confidence in a PBPK model would be high if it had reasonable biological basis, simulated the PK profile in terms of bumps and valleys for both low and high doses using a single set of parameter values and provided reliable estimates of dose metrics. The confidence in a PBPK model would be undermined if it used different values for 
a given parameter (e.g. intrinsic clearance) in order to provide adequate simulations of kinetic profiles at each dose level. Such a model would be relatively uninformative for high dose to low dose extrapolation or other risk assessment applications. In the absence of a PBPK model or another similar construct for conducting high dose to low dose extrapolation, the historical alternative has been the analysis of dose-response data on the basis of exposure doses. For example, in the case of vinyl chloride (Clewell et al., 1995), the confidence in the application of a PBPK model for the conduct of high dose to low dose extrapolation would be high based on considerations of biological basis, model performance and reliability of dose metric predictions. Here, the default linear extrapolation based on exposure dose would be highly uncertain, compared with a PBPK approach that uses a dose metric based on MOA and accounts for non-linearity of the relevant processes. This aspect has also been illustrated with dichloromethane, for which the PBPK model facilitated high dose to low dose extrapolation of the dose metric by accounting for the saturation of cytochrome P-450 (CYP) 2E1mediated metabolism and a disproportionate increase in flux through the glutathione $S$ transferase pathway with increasing doses (Andersen et al., 1987; Bos et al., 2006).

These examples emphasize the critical role and benefit of the uptake of PBPK models in risk assessment to address uncertainty associated with empirical approaches for high dose to low dose extrapolation.

\subsubsection{Route-to-route extrapolation}

The confidence in the use of PBPK models for route-to-route extrapolation will be high when evaluation of kinetics and/or dose metric is conducted for both routes in one or more species. Qualitatively, the model should be able to simulate the kinetic profile observed for each specific exposure route; on the quantitative front, the model should facilitate the simulation of the concentration-time profile in an acceptable manner (see section 3.7.1). A PBPK model that provides simulations of dose metrics based on the consideration of physiology, MOA and first-pass effects is clearly more relevant than an alternative (i.e. default) approach that assumes $100 \%$ absorption for the exposure routes. The uncertainty associated with the default (or "uninformed") route-to-route extrapolation approaches could be quite large; for example, the absorbed dose might actually be anywhere between $0 \%$ and $100 \%$. The degree of uncertainty is reduced progressively along the continuum of increasingly data-informed approaches. For example, with the dermal absorption route, the extrapolation uncertainty diminishes with the use of the available information or data in the following order (adapted from van de Sandt et al., 2007):

- no data; assumption of $100 \%$ absorption;

- QSAR modelling (steady-state assumption);

- QSAR modelling (non-steady-state condition);

- in vitro and/or experimental animal studies;

- human biomonitoring/PBPK modelling.

Even though the use of these approaches for route-to-route extrapolation might vary depending upon jurisdiction, the uncertainty associated with a particular approach is due to the conceptual model, parameters used as well as the dose metrics. In this regard, the PBPK models can be used to extrapolate the POD from one exposure route to another or to estimate the per cent absorption for each exposure route (Krishnan \& Carrier, 2008), depending upon the risk assessment need. As illustrated with vinyl chloride (Table 4), the confidence in the use of a PBPK model (Clewell et al., 1995) to perform route-to-route extrapolation relies 
essentially upon its ability to account for route-specific phenomena, physiology (e.g. the anatomical relationships of tissues and blood flows) and biochemical principles (e.g. the Michaelis-Menten equation). As PBPK models provide predictions of internal dose associated with both exposure routes in the same experimental animal (or human), it might be that variability or uncertainty analysis would not change the conclusions based on average parameter values. In this regard, the conventional route-to-route extrapolations are based only on average values, without taking into account the distributions of exposure dose, animal physiology, route-specific absorption or contact rates.

Table 4: Application for route-to-route extrapolation: characterizing the level of confidence in a PBPK model-vinyl chloride example.

\begin{tabular}{|c|c|}
\hline Model evaluation aspect & Observation \\
\hline Biological basis & $\begin{array}{l}\text { The model parameters, structure and assumptions have reasonable } \\
\text { biological basis. }\end{array}$ \\
\hline Model simulations of data & $\begin{array}{l}\text { Model consistently simulates the time course data (i.e. bumps and } \\
\text { valleys) relating to the respiratory uptake, amount metabolized as } \\
\text { well as hepatic GSH concentrations following inhalation exposures. } \\
\text { The rat model also simulates total expired carbon dioxide, as a } \\
\text { percentage of total metabolism and as a percentage of dose, } \\
\text { following oral dosing of vinyl chloride in corn oil }(0.05-100 \mathrm{mg} / \mathrm{kg} \\
\text { body weight). Overall, the PBPK model, with a single set of input } \\
\text { parameters, is capable of reproducing (qualitatively: bumps and } \\
\text { valleys; quantitatively: within a factor of } 2) \text { the various PK data sets } \\
\text { in rats and humans. }\end{array}$ \\
\hline $\begin{array}{l}\text { Reliability (model testing, } \\
\text { uncertainty and sensitivity) }\end{array}$ & $\begin{array}{l}\text { Experimental data on dose metric (i.e. metabolite in liver) following } \\
\text { oral dosing not available for comparison with model. However, } \\
\text { model simulations of total amount metabolized and GSH depletion } \\
\text { were compared with experimental data for the inhalation route but } \\
\text { not for the oral route (lack of data). A sensitivity analysis of the } \\
\text { parameters of the PBPK model showed that there was no } \\
\text { amplification of error from inputs to outputs. An uncertainty/variability } \\
\text { analysis was conducted to evaluate the impact of parameter } \\
\text { uncertainty and variability on risk prediction. The 95th percentile of } \\
\text { the distribution of the upper confidence limit risk was approximately } \\
\text { within a factor of } 2 \text { of the mean upper confidence limit risk. }\end{array}$ \\
\hline
\end{tabular}

$\mathrm{GSH}$, glutathione

\subsection{Documentation}

The documentation of a PBPK model intended for use in risk assessment requires the inclusion of sufficient information about the model and its parameters so that an experienced modeller can accurately reproduce and evaluate its performance. In order to facilitate transparency, reproducibility and credibility, the developer should systematically document the characteristics of a PBPK model. Even though the extent of documentation might depend upon the end use, the modellers generally should provide enough details to facilitate a clear understanding of the input-output relationships (and not just a flow diagram plus a table of parameters). Accordingly, the documentation should be sufficient to facilitate an experienced modeller, expert reviewer or interested end user (i.e. a risk assessor) to evaluate the model and reproduce the input-output relationships for the dose metric of relevance to MOA. Overall, PBPK model documentation should address the following broad topics: 
- $\quad$ scope and purpose of the model;

- model structure and biological characterization;

- mathematical description of ADME;

- computer implementation and verification;

- parameter estimation and analysis;

- model validation and evaluation;

- evaluation/justification of dose metrics;

- specialized analysis, if any.

The above aspects can be captured in summary form for the risk assessment audience but in greater depth for specialists, as is typically done in technical publications. In the latter case, it is particularly important to identify clearly the data sets that were used to evaluate the model, along with the rationale for excluding data sets, if any, during model development. Similarly, the alternative model structures considered, the range of values assigned for each of the input parameters as well as the exposure conditions for sensitivity, uncertainty and variability analyses should be presented along with the rationale. For risk assessment application, the original model code, corresponding to the published manuscript, is essential and should be provided to the regulatory scientists for independent evaluation and reproduction of any simulations that form the basis of dose metrics used in the risk assessment. Supporting files and data sets sufficient to reproduce published plots (comparing the model simulations with the experimental data) and reported numerical results (exposure/dose calculations) should also be submitted to the regulatory scientists. Further justification of the dose metric on the basis of plausibility and consistency with available information on MOA as well as doseresponse information for the critical effect should also be presented and necessarily requires input from a range of multidisciplinary experts (see section 4.3 for further discussion; also see IPCS, 2005a).

\section{APPLICATION OF PBPK MODELS IN RISK ASSESSMENT}

Application of PBPK models shifts the focus from the administered dose to a measure of internal dose that is more closely associated with the toxic response. For enhancing the scientific basis of risk assessments, PBPK models can be used to obtain predictions of dose metrics of relevance to the MOA of chemicals. Such predictions can be obtained not only for the POD (e.g. NOAEL) but also for a variety of study doses for enhancing the modelling of the dose-response data. It is therefore critical that a PBPK model intended for use in risk assessment be able to adequately simulate the dose metrics (potentially or causally related to toxic responses) for the exposure route, dose ranges, life stage and species used in the critical studies, as well as for anticipated human exposures.

The guiding principles for PBPK-based risk assessments are discussed in sections 4.1-4.4 in terms of the following aspects: 1) choice of critical studies; 2) selection of PBPK models; 3 ) evaluation of dose metrics and 4) determination of human exposures.

\subsection{Choice of critical studies}

The starting point for performing a risk assessment using PBPK models is essentially the same as for the conventional approach - that is, evaluate the available toxicological, epidemiological and mechanistic data for the chemical and identify potentially useful critical 
studies. In an assessment based on PBPK models, as in any other systematic assessment of toxicological and epidemiological data, more than one critical study (i.e. a study that predicts effects at the lowest dose) may be retained for identifying the POD. This is a consequence of the fact that dose equivalence across species for different toxicological end-points cannot be predicted a priori and would depend upon the dose metrics (consistent with the MOA) to be simulated with the use of PBPK models (Gentry et al., 2004). Once the critical studies are identified, the required capability of PBPK models in terms of the species, life stages, dose ranges, exposure routes and end-points (dose metrics and target tissues) would be clear.

\subsection{Selection of PBPK models}

PBPK models are selected to be consistent with the critical studies identified in section 4.1, as well as the objectives of the risk assessment. Box 2 presents a checklist of key aspects to be considered in selecting chemical-specific PBPK models for a given risk assessment.

A model whose scientific validity has been established through peer review and is capable of providing reliable dose metric simulations may not be applicable in a risk assessment if it is not "fit for the purpose". The intended application in risk assessment (e.g. evaluating interspecies differences, characterizing population distributions, route-to-route extrapolation) essentially determines the desired model characteristics. For example, if a critical study was conducted in adult animals, then, for conducting interspecies extrapolation, the appropriate PBPK model would have been developed and calibrated for:

- adult test species and adult humans;

- exposure routes used in the critical toxicological study as well as the exposure matrix and route of relevance to risk assessment (e.g. air: inhalation); and

- providing simulations of not only parent chemical but also metabolites (if applicable) in target tissues or a surrogate compartment (e.g. blood).

Here the models may be deterministic in nature, based on average parameter values for the test animals and humans, as with the case-study example in Annex 3. In contrast, if the PBPK model is intended for use in quantifying human variability in dose metrics, it would have been developed and calibrated for:

- human populations (i.e. characterized by distributions of parameters);

- exposure routes of relevance to risk assessment (e.g. air: inhalation); and

- simulating population distributions of not only parent chemical but also metabolites (if applicable) in target tissues or a surrogate compartment (e.g. blood).

In this regard, probabilistic PBPK modelling, based on the Monte Carlo simulation approach, alone or in conjunction with the Markov chain Monte Carlo method, may be used to generate population distributions of dose metrics (Bernillon \& Bois, 2000; Jonsson \& Johanson, 2001, 2002; Willmann et al., 2007).

For conducting route-to-route extrapolations with PBPK models, one of two approaches is used: 1) an animal model is used to extrapolate a POD from one route to another on the basis of an equivalent dose metric or 2) test animal and human PBPK models for a chemical are used to determine $\mathrm{POD}_{\text {human }}$ for one route from the available $\mathrm{POD}_{\text {animal }}$ for another route on the basis of an equivalent dose metric (Chiu et al., 2007). The extrapolation of the dose 


\section{Box 2: Checklist of characteristics for selecting PBPK models for use in risk assessment}

\section{Biological basis}

- Are the major sites/processes of absorption, storage, transformation and clearance included in the model?

- Are the mathematical equations of ADME based on a sound theoretical or biological basis?

- Are the input parameters related to the characteristics of the host, chemical or environment?

- Is the sum total of the tissue blood flow rates equal to the cardiac output?

- Is the ventilation:perfusion ratio specified in the model within physiological limits?

- Are the volumes of compartments (individual and total) within known physiological limits?

- Is the approach used to establish partition coefficients within the domain of valid application?

- Is the method used for estimating biochemical parameters adequate?

- Is the allometric scaling of parameters, if applicable, done appropriately?

- Is the integration algorithm proven for solving differential equations in similar models?

- Has the computer model code been verified for syntax errors and the accuracy of units (i.e. dimensional consistency)?

\section{Model simulation of data}

- Has the model been evaluated for its ability to predict kinetics under various conditions, consistent with its intended application?

- Does the model consistently reproduce the general trend of the data (i.e. peaks, bumps and valleys, saturation of metabolism) or only portions of one or more data sets?

- Are the model predictions within an acceptable level of correspondence with the experimental data (e.g. within a factor of 2)?

Reliability (model testing, uncertainty and sensitivity)

- Is the model capable of providing predictions of the concentration time course of the candidate dose metrics in the target organ or a suitable surrogate compartment (e.g. blood)?

- Has the uncertainty in model predictions of dose metric been assessed for the relevant exposure conditions?

- What is the reliability of the data used for calibrating and/or evaluating the PBPK model?

- Is the sensitivity of the dose metric to change in numerical values of input parameters characterized for relevant exposures?

\section{Applicability}

- Has the model been developed and evaluated in the species and life stage of relevance to the risk assessment?

- Do the exposure routes in the model correspond to those of anticipated human exposures as well as those of the critical studies chosen for the assessment?

- Has the model been tested for the exposure doses and durations of relevance to the intended extrapolations?

- Does the model contain point estimates (or distributions) of parameters, consistent with the purpose of application?

metric of a chemical from one exposure route to another is performed by including appropriate equations to represent each exposure pathway (Figure 16). In this context, then, appropriate PBPK models would have been developed and calibrated for: 
- adult test animals and/or humans (or another relevant life stage);

- simulating the kinetics of chemicals for the two routes of relevance (i.e. the exposure route for which the POD is available and the route for which extrapolation is intended); and

- providing simulations of not only parent chemical but also metabolites (if applicable) in target tissues or a surrogate compartment (e.g. blood) by both routes of exposure.

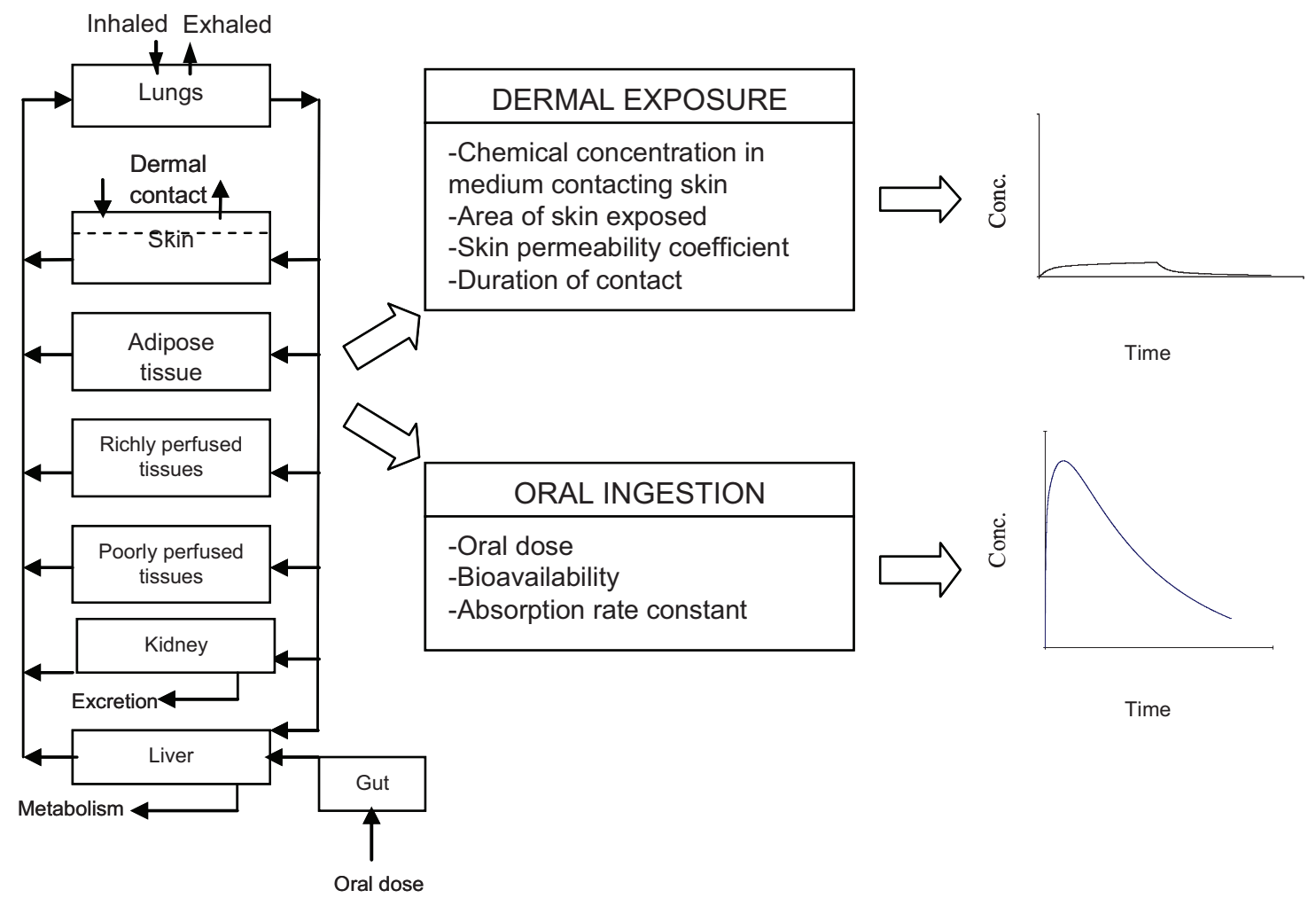

STRUCTURE

ROUTE-SPECIFIC PARAMETERS

SIMULATIONS

Figure 16: Illustration of the conceptual approach for the application of PBPK models in the conduct of route-to-route (oral-to-dermal) extrapolation.

Again, these models may be deterministic in nature, based on average parameter values for the test animals and humans, as with the case-study chemical VC (see Annex 3).

When there is more than one model that conforms to the above criteria, it then becomes a question of which one among the alternative models would be the most appropriate for use. In this regard, it is important to assess how well the particular models have been evaluated for their reliability in predicting the candidate dose metrics. Statistical comparisons of PBPK model structures in relation to the data could be carried out; however, the goal should be to minimize the uncertainty in prediction of the dose metric rather than to obtain the best fit to all available data. Based on considerations of the conformity of the model predictions to experimental data, the biological basis of the models as well as their ability to reliably predict dose metrics of relevance to MOA, the appropriate model is chosen for risk assessment application. 


\subsection{Evaluation of dose metrics}

The dose metric required for the conduct of PBPK-based risk assessments relates to the form of chemical (e.g. parent chemical or metabolite), its level (concentration or amount), duration (instantaneous, daily, lifetime or a specific developmental period), intensity (peak, average or integral) as well as the biological matrix (e.g. blood, target tissue) that is consistent with the MOA of the chemical (USEPA, 2006). The circulating level of the active form of chemical is useful as a basis for PK extrapolations and comparisons (IPCS, 2005a). More specifically, for chronic non-carcinogenic effects, the AUC in target tissue or surrogate compartment (e.g. blood), determined as the daily average, has often been used as the dose metric (Collins, 1987; Voisin et al., 1990; Clewell et al., 2002). For carcinogens producing reactive intermediates, the amount of metabolite produced per unit time and the amount of metabolite in target tissue over a period of time (e.g. milligrams of metabolite per litre of tissue during 24 h) have been used as dose metrics (Andersen et al., 1987; Andersen \& Dennison, 2001). For developmental effects, the dose metric is defined in the context of the window of exposure for a particular gestational event (e.g. Welsch et al., 1995). For some compounds, dose metrics based upon free concentrations are more appropriate than those based upon total concentrations (Kalvass \& Maurer, 2002). Other metrics of tissue exposure may also be appropriate for risk assessment purposes and determined on the basis of the current state of knowledge of the MOA of chemicals (IPCS, 2005a).

Typically, the PBPK model is used to calculate several potentially plausible dose metrics for each critical study and end-point selected. The specific nature of the dose metric calculations to be performed with PBPK models would depend upon the nature of the assessment. For example, in the case of a non-cancer assessment based on a NOAEL, the dose metric would be calculated only for the NOAEL for a particular critical study and end-point. In contrast, if the assessment uses the BMD approach, then dose metrics associated with each of the treatment groups would be calculated (Gentry et al., 2004). It is important to use the appropriate simulation period to characterize the dose metrics associated with test animal and human exposures, consistent with the knowledge on the MOA of the chemical. For example, if toxicological measurements (e.g. neurobehavioural measures) are done at a specific time following exposure, the dose metric should reflect internal exposures only up to the time of that measurement. In this case, if measurements were done at several times during a day, the peak concentration might be the same for all of them, but the AUC would increase with later measurements. The dose metric associated with human exposures should be calculated in just the same way as the dose metric for the critical toxicity study. For example, if the dose metric in the experimental animal toxicity study was expressed in terms of area under the free concentration versus time curve during $24 \mathrm{~h}$, then the dose metric for human exposure should also correspond to free $\mathrm{AUC}_{24} \mathrm{~h}$. Alternatively, if it is the peak concentration during a $24 \mathrm{~h}$ period, then the same measure should be obtained in both species.

It is important that the proposed MOA and dose metric be adequately justified to elicit confidence in the PBPK model-based risk assessment. When there are several candidate dose metrics, the appropriate one for use in risk assessment should be chosen on the basis of plausibility. The plausibility of a particular dose metric is determined by its consistency with available information on the MOA as well as dose-response information for the end-point of concern. In this regard, the chosen dose metric would show a consistent quantitative relationship with the levels of responses seen in a single study (internal consistency) as well as the other available studies regardless of differences in exposure scenario, route and species (external consistency) (Clewell et al., 2002). If multiple chemicals act through a specific 
MOA, support for selection of the appropriate dose metric may be obtained from another chemical with a larger database. If the analyses fail to identify the most appropriate dose metric, the results can be used to characterize the range of uncertainty associated with the output of the analysis, where feasible (Gentry et al., 2004). The last resort, when any one dose metric among the possible ones cannot be selected (on the basis of resolution of differences and inconsistencies across studies and dose groups), might involve the use of the dose metric yielding the highest risk estimate or lowest acceptable human exposure level.

The dose metrics generated using the PBPK models are then used in the dose-response model in lieu of exposure concentrations or exposure doses, to conduct various extrapolations for deriving the human exposure values (Andersen et al., 1987; Clewell et al., 2002; Thompson et al., 2008).

\subsection{Determination of human exposures}

Conventionally, in risk assessments of threshold toxicants, the human-equivalent dose is determined by dividing the $\mathrm{POD}_{\text {animal }}$ by the uncertainty factors $\left(\mathrm{AK} \mathrm{UF}_{\mathrm{UF}}, \mathrm{AD}_{\mathrm{UF}}\right)$ or $\mathrm{CSAFs}$ $\left(\mathrm{AK}_{\mathrm{AF}}, \mathrm{AD}_{\mathrm{AF}}\right)$. To the extent that PBPK models are viewed as tools for estimating CSAFs, the model can be used to estimate the dose metric associated with the POD in both the test animal species and humans and then divide one by the other to determine the magnitude of the CSAF. However, in a PBPK-based risk assessment, it is only appropriate to divide the dose metrics obtained from the toxicity studies by the remaining factors rather than applying them to the exposure concentrations or doses (Figure 17). The application of assessment factors to the exposure dose in the test species might lead to erroneous or questionable human doses, particularly if the relationship between the internal dose and applied dose is non-linear in the range of doses used in the toxicological studies. Therefore, the dose metrics should be adjusted to ensure that the biologically effective dose is reduced to the extent desired, before deriving the human doses. Accordingly, an $\mathrm{AD}_{\mathrm{UF}}$ applied in PBPK-based assessment corresponds to 2.5, accounting for the remaining uncertainty in TD differences across species; however, in specific cases, the actual value may be lower or greater than 2.5 , based on other available data on the relative sensitivity of humans compared with the test animal species (IPCS, 2005a). The process of implementation of PBPK models in the determination of human exposure concentrations or doses from test animal data can be summarized as follows (Clewell et al., 2002; Gentry et al., 2004; Thompson et al., 2008):

- A PBPK model is selected (section 4.2) and used to simulate dose metrics associated with the doses used for characterizing the POD or the dose-response relationship (section 4.3) in the test species.

- The dose metric associated with the POD is then divided by the appropriate assessment or uncertainty factors.

- The PBPK model, following replacement of parameters with those corresponding to a typical human, is used to determine the safe exposure concentration or dose associated with the target dose metric identified above. In this process, the PBPK model is run repeatedly by varying the human exposure concentration or dose until the target dose metric value is obtained.

It is relevant to note that the PBPK model can be used with both NOAEL and BMDL approaches to translate the study doses to dose metrics to which dose-response modelling may then be applied. For example, guideline values for 2-butoxyethanol and vinyl chloride have been derived with the use of PBPK models (USEPA, 2000, 2010; Environment Canada 
\& Health Canada, 2002). Additional examples of PBPK application in the risk assessment of carcinogens and systemic toxicants have been summarized by DeWoskin et al. (2007). Two distinct characteristics regarding the application of uncertainty factors in PBPK-based risk assessments are as follows (Gentry et al., 2004):

1. The uncertainty factors are applied to the dose metric and not to the exposure concentration or applied dose.

2. Only a portion of the uncertainty factor that corresponds to the pharmacodynamic uncertainty $\left(\mathrm{AD}_{\mathrm{UF}}, \mathrm{HD}_{\mathrm{UF}}\right)$ is applied if PBPK models for both test species and humans are used.

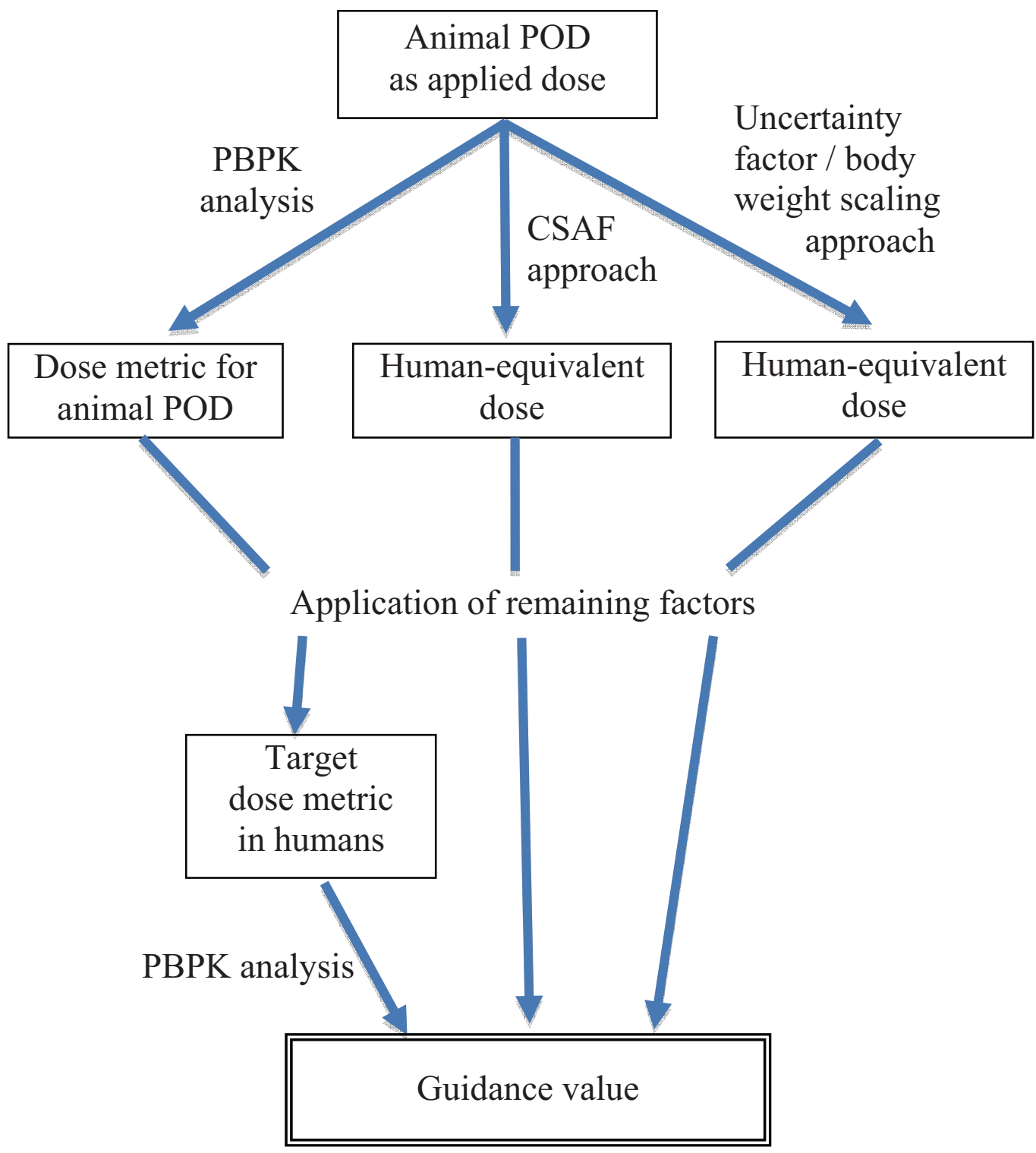

Figure 17: The application of assessment factors in the derivation of guidance values according to different approaches. In this particular example, the remaining factors refer to the interspecies TD uncertainty as well as human variability in TK and TD. Note that the CSAFs can be derived from empirical data, PK parameters or PBPK models. 


\section{PROCESS CONSIDERATIONS}

Stimulating the dialogue between modellers and risk assessors, without compromising the integrity of the process, is likely to enhance the application of PBPK models in risk assessment. Optimally, the development of a model for application in risk assessment would involve upfront consultation between the modeller and the risk assessor. Particularly the definition of the scope for model use and the capability of the model can be determined by the consideration of MOA and pathways of exposure, with the involvement of the risk assessor. The development of PBPK models without such two-way interactions - at any level - may ultimately result in an end product that may not be relevant or applicable for risk assessment purposes. In order to maximize the development of PBPK models consistent with the end use (i.e. risk assessment), the following paradigms are useful (Loizou et al., 2008):

- Continuous involvement: Here, the risk assessor is involved right from the beginning in the model formulation and model evaluation processes. This permits the modeller and risk assessor to jointly consider the data on MOA and identify dose metrics of relevance. The interaction between the risk assessor and modeller might continue through the evaluation of the dose-response data. Such an ongoing feedback mechanism would enable the modeller to consider and address issues of relevance to the MOA and risk assessment at every step of the process, such that the resulting model is useful for addressing the key issues and uncertainties in an assessment.

- Iterative involvement: The risk assessor provides input at specific stages of model development and evaluation. Depending upon the stage of the involvement, the risk assessor may or may not have an impact on the end product or its relevance. In this regard, the dialogue between the risk assessor and the modeller can be helpful in identifying data gaps and prioritizing avenues for additional research, including aspects of model development, particularly when a published model fails to address the issues at hand.

To the extent possible, the development of PBPK models intended for use in risk assessment would be undertaken with continuous involvement of a risk assessor from the problem formulation stage. This value-added feedback mechanism involving the risk assessor in the process, however, should in no way compromise PBPK model development or evaluation as an "independent" process.

The application and acceptance of PBPK models in risk assessment would depend upon access to PBPK expertise within the regulatory environment such that adequate evaluation of PBPK models and their output can be carried out. The following sections identify some key aspects regarding the expertise, training and communication efforts required to achieve the goals related to the optimal use of PBPK models in risk assessment.

\subsection{Expertise}

Based on the scope of and the identified need for a PBPK model in an assessment, the implementation process would require personnel with expertise to perform the following four tasks: 
1. review and selection of critical toxicological studies;

2. review and selection of a PBPK model for the chemical in question;

3. evaluation of dose metrics, based on plausibility and MOA considerations; and

4. determination of health-based guidance values (e.g. RfD/RfC, ADI/TDI).

Whereas the risk assessor would play a leading role in steps 1 and 3 and additionally be involved in each of the other steps, the modeller as well as a statistician would play critical roles in steps 2 through 4. Specifically, step 2 would benefit from peer engagement for ensuring the integrity of the PBPK model and its reliability to simulate dose metrics of relevance to the risk assessment. Specifically, an international standing committee might be constituted to provide a support structure for providing continuous feedback on key aspects regarding the credibility and reliability of PBPK models.

Risk assessors have traditionally received graduate training in pharmacology, toxicology and related biomedical sciences and therefore possess broad knowledge in a number of areas, including toxicokinetics, xenobiotic metabolism, physiology, target organ toxicology and dose-response modelling. While expertise in these areas is fundamentally useful for the understanding of the biological and mechanistic basis of PBPK models, additional expertise in applied mathematics, statistics and computational methods is required in order to appropriately evaluate and apply these models in risk assessment. The latter expertise, of course, would also facilitate a better understanding of the more sophisticated mathematical models of human exposure, environmental fate and risk characterization. Development of training materials and hiring of personnel with appropriate expertise will be essential to augment the implementation of MOA- and PBPK-based risk assessment by regulatory agencies.

\subsection{Training}

Training in biological and life sciences is evolving significantly with an orientation towards quantitative analysis and modelling, as opposed to the historical focus on qualitative aspects. A number of public and private institutions as well as professional societies in Europe and North America have periodically been conducting hands-on training sessions on PBPK modelling. Such focused training, offered in the form of continuing education courses, workshops or short courses, would be relevant to end users by providing a unique opportunity:

- to understand how the information on toxicology and MOA is integrated within the model development process;

- to see how the equations are written, compiled and solved using commercially available software;

- to learn from case-studies of application of PBPK models in risk assessment; and

- to learn about current or newer methodologies for characterizing uncertainty and variability in PBPK models.

A number of software packages (e.g. MEGen, Berkeley Madonna, acslXtreme, Matlab) are helpful, as they permit the development of generic PBPK models for demonstrating to students how physiological and biochemical information is integrated to address issues and uncertainty related to specific toxicology and risk assessment problems. Such generic models as well as step-by-step instructions may be assembled to develop a focused, web-based 
training (and retraining) package that is easily accessible by risk assessors and other interested audiences.

Even though such short-term or web-based training materials on PBPK modelling and its applications in risk assessment can help prepare individuals lacking quantitative skills and expertise, the longer-term goal should be to include a more quantitative, computationally based study of toxicology in university curricula.

\subsection{Communication}

Efficient communication is centrally important in facilitating practical application of PBPK models in risk assessment. The issues related to communication between modellers and risk assessors are somewhat different from those encountered in the context of communication within the risk assessment community or within the modelling community. The communication from the risk assessor to the modeller on aspects of the risk assessment issues, scope for model use and MOA and dosimetric considerations is central to making progress in the uptake of PBPK models. Some key aspects that require consideration in communication between modellers and risk assessors are as follows:

- Development of model descriptions in a standard format to effectively transfer information about the model, the data supporting it and the appropriateness of its application under a range of conditions. To this end, a template for PBPK model description is presented in Box 3; a case-study (and an expanded template) is presented in Annex 3 to illustrate the effective use of this template by modellers to communicate the essential elements to the risk assessors.

\section{Box 3: Template for describing the characterization and application of PBPK} models in risk assessment

1. BACKGROUND

1.1 Critical effect

1.2 Pharmacokinetics

1.3 Mode of action/relevant dose metric

1.4 Scope for PBPK model application

\section{PBPK MODEL: CHARACTERIZATION AND EVALUATION}

2.1 Model capability and selection

2.2 Model structure and biological characterization

2.3 Parameter estimation and analysis

2.4 Purpose-specific model evaluation

2.5 Model documentation

2.6 Model peer review

3. PBPK MODELLING AND EVALUATION OF DOSE METRICS

4. PBPK MODEL APPLICATION AND COMPARISON WITH DEFAULT

- Presentation of key model parameters along with the methodologies used is critical to a better communication and understanding of the model development elements by the 
assessors (see Table B1.1 in Annex 3). It is also important to identify which of the key parameters were developed based on fitting to PK data sets and whether the data used for model evaluation were different from those used for model calibration (i.e. fitting). In cases where the fitting or re-estimation of model parameters is undertaken for simulating the kinetics in various age groups, sexes and exposure conditions, it is more useful to illustrate the overall process using a flow diagram or another such pictorial (e.g. Clewell et al., 2008). For example, Figure 18 illustrates the process of parameter estimation for the inhalation PBPK model of the chemical 2-butoxyethanol in male rats (Corley et al., 1994; Lee et al., 1998).

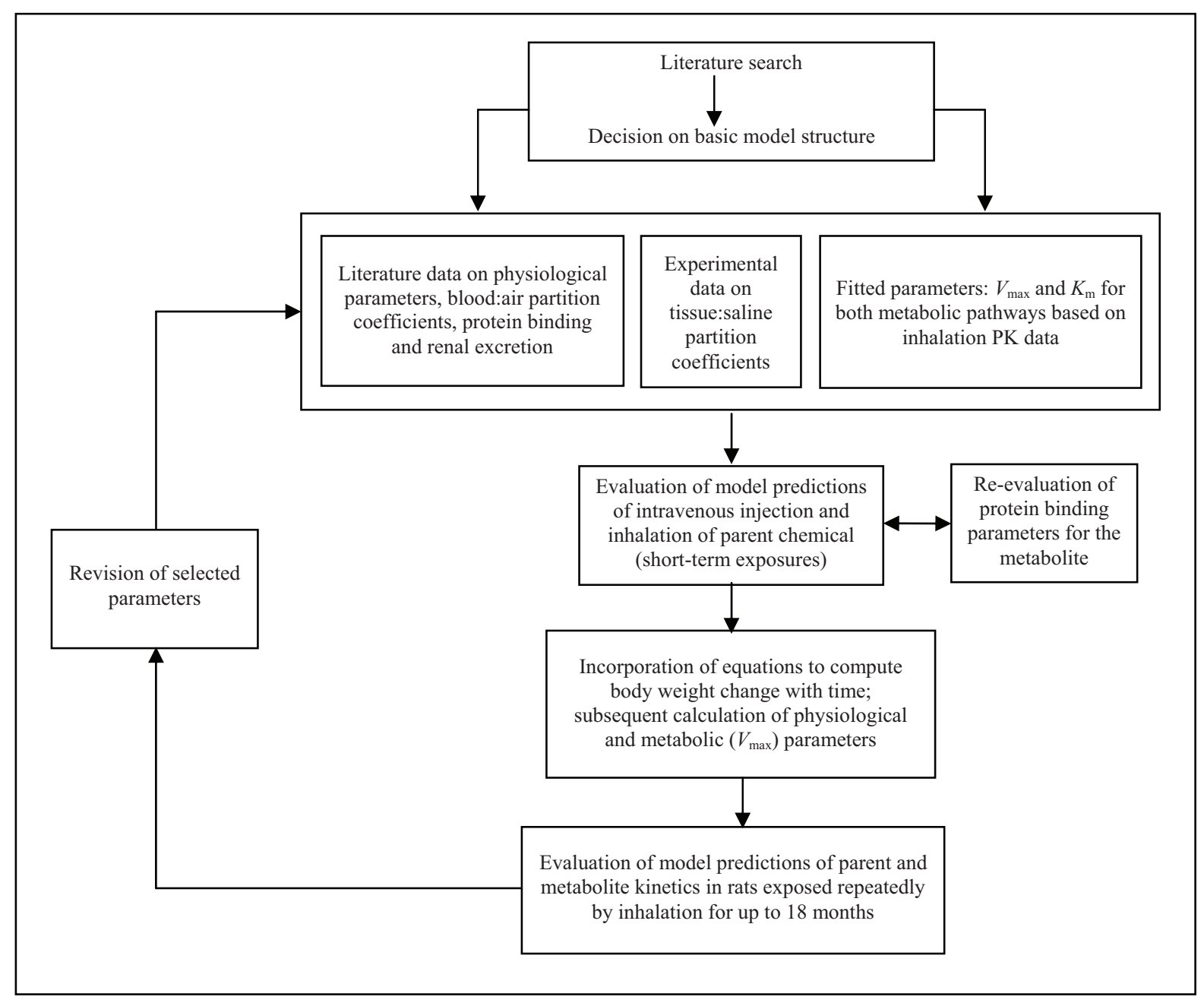

Figure 18: Simplified illustration of the model development process for 2-butoxyethanol in male rats (based on Lee et al., 1999).

- Such illustrations, combined with the parameter tables, would be instrumental in communicating the model development aspects, with particular emphasis on the parameter estimation processes.

- Communicative pieces illustrating the critical importance of "purpose-specific" model evaluation should be developed. A common impediment to the use of PBPK models in risk assessment relates to the criticism or confusion regarding the extent of validation or evaluation required. As illustrated with chemical VC in the case-study (see Annex 3), the 
focus should be on "fit for purpose". Accordingly, it is important to present clearly the level of confidence in a model based on its performance, biological basis and reliability as they relate to a specific purpose or application in risk assessment.

- Establishment of standard abbreviations or parameter nomenclature as well as a glossary for PBPK modelling is central to facilitate communication not only between risk assessors and modellers, but also among modellers themselves. In this regard, a library of generic model structures, descriptions of departures from the generic structure as well as lessons learnt regarding the evaluation of dose metrics for specific MOAs might be envisaged.

\section{CONCLUDING REMARKS}

- PBPK models provide a documentable and scientifically defensible means of bridging the gap between critical toxicity studies and human risk estimates by facilitating interspecies, interindividual, high dose to low dose and route-to-route extrapolations. In particular, they shift the focus in risk assessments from external dose to internal dose, which is more closely associated with the tissue responses. The PBPK models, however, will not remove all of the uncertainty associated with the risk assessment process; specifically, in most cases, they would not address TD uncertainty.

- The complexity of PBPK models should be no more than is required for the task at hand. The increased complexity and data demands of PBPK models must be counterbalanced by the increased accuracy, biological basis and scientific justifiability of the risk assessment using them. While complex PBPK models may be relevant to chemicals for which margin between exposure and effect is small, simpler models might be adequate for preliminary assessments to inform additional steps.

- For PBPK models intended for application in risk assessment, the focus should be on purpose-specific "evaluation" rather than generic "validation". Accordingly, comparison of model predictions with PK data is not the only way of establishing confidence in a PBPK model; equally important are aspects relating to the biological basis of the model structure and parameters as well as the reliability of dose metric predictions, supplemented with appropriate analyses of variability, uncertainty and sensitivity.

- In order to facilitate transparency, reproducibility and credibility, the PBPK models should be systematically characterized and documented. The documentation should be sufficient to enable an experienced modeller, expert reviewer or interested end user to evaluate a PBPK model and reproduce the input-output relationships for the dose metric of relevance to the risk assessment. Transparency could be improved through development of dedicated repositories for data, models and their detailed documentation.

- Communication between the modeller and the risk assessor is of prime importance in developing PBPK models applicable for risk assessment. The continuous involvement of a risk assessor right from the problem formulation stage would be key in helping the modeller consider and address issues of relevance to MOA and risk assessment. 
- Mechanisms for adequate peer engagement at the international level for evaluating PBPK models in the context of their suitability for specific applications in risk assessment would be essential. For example, an international steering committee or a peer review group could facilitate such a process. This, in conjunction with enhanced access to modelling expertise through recruitment, training or retraining, would facilitate greater uptake and optimal use of PBPK models by the risk assessment community. 


\section{REFERENCES}

Adolph EF (1949) Quantitative relations in the physiological constitutions of the mammals. Science, 109:579-585.

Allen BC, Covington TR, Clewell HJ (1996) Investigation of the impact of pharmacokinetic variability and uncertainty on risks predicted with a pharmacokinetic model for chloroform. Toxicology, 111:289-303.

Andersen ME (2003) Toxicokinetic modeling and its applications in chemical risk assessment. Toxicology Letters, 138(1):9-27.

Andersen ME, Dennison JE (2001) Mode of action and tissue dosimetry in current and future risk assessments. Science of the Total Environment, 274:3-14.

Andersen ME, Clewell HJ, Gargas ML, Smith FA, Reitz RH (1987) Physiologically based pharmacokinetics and the risk assessment process for methylene chloride. Toxicology and Applied Pharmacology, 87(2):185-205.

Andersen ME, Clewell HJ, Gargas ML (1991) Physiologically-based pharmacokinetic modeling with dichloromethane, its metabolite carbon monoxide and blood carboxyhemoglobin in rats and humans. Toxicology and Applied Pharmacology, 108:14-27.

Andersen ME, Clewell HJ, Frederick CB (1995a) Applying simulation modeling to problems in toxicology and risk assessment - $\mathrm{a}$ short perspective. Toxicology and Applied Pharmacology, 133(2):181-187.

Andersen ME, Clewell HJ, Krishnan K (1995b) Tissue dosimetry, pharmacokinetic modeling, and interspecies scaling factors. Risk Analysis, 15:533-537.

Arms AD, Travis CC (1988) Reference physiological parameters in pharmacokinetic modeling. Washington, DC, United States Environmental Protection Agency, Office of Health and Environmental Assessment (NTIS PB 88-196019).

Balci O (1997) Verification, validation and accreditation of simulation models. In: Andradottir S, Healy KJ, Withers DH, Nelson BL, eds. Proceedings of the 1997 Winter Simulation Conference. San Diego, CA, Society for Computer Simulation, pp. 135-141.

Barter ZE, Bayliss MK, Beaune PH, Boobis AR, Carlile DJ, Edwards RJ, Houston JB, Lake BG, Lipscomb JC, Pelkonen OR, Tucker GT, Rostami-Hodjegan A (2007) Scaling factors for the extrapolation of in vivo metabolic drug clearance from in vitro data: reaching a consensus on values of human microsomal protein and hepatocellularity per gram of liver. Current Drug Metabolism, 8:33-45.

Barton HA, Flemming CD, Lipscomb JC (1996) Evaluating human variability in chemical risk assessment: hazard identification and dose-response assessment for noncancer oral toxicity of trichloroethylene. Toxicology, 111:271-287.

Barton HA, Chiu WA, Setzer RW, Andersen ME, Bailer AJ, Bois FY, Dewoskin RS, Hays S, Johanson G, Jones N, Loizou G, Macphail RC, Portier CJ, Spendiff M, Tan YM (2007) 
Characterizing uncertainty and variability in physiologically-based pharmacokinetic (PBPK) models: state of the science and needs for research and implementation. Toxicological Sciences, 99(2):395-402.

Béliveau M, Lipscomb JC, Tardif R, Krishnan K (2005) Quantitative structure-property relationships for interspecies extrapolation of the inhalation pharmacokinetics of organic chemicals. Chemical Research in Toxicology, 18:475-485.

Bernillon P, Bois FY (2000) Statistical issues in toxicokinetic modeling: a Bayesian perspective. Environmental Health Perspectives, 108(5):883-893.

Bogdanffy MS, Sarangapani R, Plowchalk DR, Jarabek A, Andersen ME (1999) A biologically based risk assessment for vinyl acetate-induced cancer and noncancer inhalation toxicity. Toxicological Sciences, 51(1):19-35.

Bogdanffy MS, Plowchalk DR, Sarangapani R, Starr TB, Andersen ME (2001) Mode-ofaction-based dosimeters for interspecies extrapolation of vinyl acetate inhalation risk. Inhalation Toxicology, 13(5):377-396.

Bois FY (2000a) Statistical analysis of Clewell et al. PBPK model of trichloroethylene kinetics. Environmental Health Perspectives, 108(2):307-316.

Bois FY (2000b) Statistical analysis of Fisher et al. PBPK model of trichloroethylene kinetics. Environmental Health Perspectives, 108(2):275-282.

Bois FY (2001) Applications of population approaches in toxicology. Toxicology Letters, 120(1-3):385-394.

Boobis AR, Cohen SM, Dellarco V, McGregor D, Meek ME, Vickers C, Willcocks D, Farland W (2006) IPCS framework for analyzing the relevance of a cancer mode of action for humans. Critical Reviews in Toxicology, 36:781-792.

Boobis AR, Doe JE, Heinrich-Hirsch B, Meek ME, Munn S, Ruchirawat M, Schlatter J, Seed J, Vickers C (2008) IPCS framework for analysing the relevance of a non-cancer mode of action for humans. Critical Reviews in Toxicology, 38(2):87-96.

Bos PM, Zeilmaker MJ, van Eijkeren JC (2006) Application of physiologically based pharmacokinetic modeling in setting acute exposure guideline levels for methylene chloride. Toxicological Sciences, 91(2):576-585.

Brightman FA, Leahy DE, Searle GE, Thomas S (2006) Application of a generic physiologically based pharmacokinetic model to the estimation of xenobiotic levels in rat plasma. Drug Metabolism and Disposition, 34(1):84-93.

Brown RP, Delp MD, Lindstedt SL, Rhomberg LR, Beliles RP (1997) Physiological parameter values for physiologically based pharmacokinetic models. Toxicology and Industrial Health, 13:407-484. 
Campolongo F, Saltelli A (1997) Sensitivity analysis of an environmental model: an application of different analysis methods. Reliability Engineering and System Safety, 57(1):49-69.

Carson ER, Cobelli C, Finkelstein L (1983) The mathematical modeling of metabolic and endocrine systems: model formulation, identification, and validation. New York, NY, John Wiley \& Sons.

Chiu WA, Barton HA, Dewoskin RS, Schlosser P, Thompson CM, Sonawane B, Lipscomb JC, Krishnan K (2007) Evaluation of physiologically based pharmacokinetic models for use in risk assessment. Journal of Applied Toxicology, 27:218-237.

Clark LH, Setzer RW, Barton HA (2004) Framework for evaluation of physiologically-based pharmacokinetic models for use in safety or risk assessment. Risk Analysis, 24(6):1697-1717.

Clewell HJ, Andersen ME (1985) Risk assessment extrapolations and physiological modeling. Toxicology and Industrial Health, 1(4):111-131.

Clewell HJ III, Andersen ME (1987) Dose, species, and route extrapolation using physiologically based pharmacokinetic models. In: Pharmacokinetics in risk assessment: drinking water and health. Vol. 8. Washington, DC, National Academy Press, pp. 159-184.

Clewell HJ, Lee T, Carpenter RL (1994) Sensitivity of physiologically based pharmacokinetic models to variation in model parameters: methylene chloride. Risk Analysis, 14:521531.

Clewell HJ, Gentry PR, Gearhart JM, Allen BC, Andersen ME (1995) Considering pharmacokinetic and mechanistic information in cancer risk assessments for environmental contaminants: examples with vinyl chloride and trichloroethylene. Chemosphere, 31(1): 2561-2578.

Clewell HJ, Gearhart JM, Gentry PR, Covington TR, VanLandingham CB, Crump KS, Shipp AM (1999) Evaluation of the uncertainty in an oral reference dose for methylmercury due to interindividual variability in pharmacokinetics. Risk Analysis, 19:547-558.

Clewell HJ, Andersen ME, Barton HA (2002) A consistent approach for the application of pharmacokinetic modeling in cancer and noncancer risk assessment. Environmental Health Perspectives, 110:85-93.

Clewell HJ, Gentry PR, Covington TR, Sarangapani R, Teeguarden JG (2004) Evaluation of the potential impact of age- and gender-specific pharmacokinetic differences on tissue dosimetry. Toxicological Sciences, 79(2):381-393.

Clewell RA, Clewell HJ III (2008) Development and specification of physiologically based pharmacokinetic models for use in risk assessment. Regulatory Toxicology and Pharmacology, 50(1):129-143.

Clewell RA, Kremer JJ, Williams CC, Campbell JL Jr, Andersen ME, Borghoff SJ (2008) Tissue exposures to free and glucuronidated monobutylphthalate in the pregnant and fetal rat 
following exposure to di- $n$-butylphthalate: evaluation with a PBPK model. Toxicological Sciences, 103(2):241-259.

Cobelli C, Carson ER, Finkelstein L, Leaning MS (1984) Validation of simple and complex models in physiology and medicine. American Journal of Physiology, 246:R259-266.

Collins JM (1987) Prospective predictions and validations in anticancer therapy. In: Pharmacokinetics in risk assessment: drinking water and health. Vol. 8. Washington, DC, National Academy Press, pp. 431-440.

Corley RA, Bormett GA, Ghanayem BI (1994) Physiologically based pharmacokinetics of 2butoxyethanol and its major metabolite, 2-butoxyacetic acid, in rats and humans. Toxicology and Applied Pharmacology, 129(1):61-79.

Corley RA, Mast TJ, Carney EW, Rogers JM, Daston GP (2003) Evaluation of physiologically based models of pregnancy and lactation for their application in children's health risk assessments. Critical Reviews in Toxicology, 33(2):137-211.

Davies B, Morris T (1993) Physiological parameters in laboratory animals and humans. Pharmaceutical Research, 10:1093-1095.

Dedrick RL, Bischoff KB (1980) Species similarities in pharmacokinetics. Federation Proceedings, 39:54-59.

Dedrick RL, Forrester DD, Ho DHW (1972) In vitro-in vivo correlation of drug metabolism: deamination of 1- $\beta$-D-arabinosyl cytosine. Biochemical Pharmacology, 21:1-16.

Dedrick RL, Zaharko DS, Lutz RJ (1973) Transport and binding of methotrexate in vivo. Journal of Pharmaceutical Sciences, 62:882-890.

DeWoskin RS, Lipscomb JC, Thompson C, Chiu WA, Schlosser P, Smallwood C, Swartout J, Teuschler LK, Marcus A (2007) Pharmacokinetic/physiologically based pharmacokinetic models in integrated risk information system assessments. In: Lipscomb JC, Ohanian EV, eds. Toxicokinetics and risk assessment. New York, NY, Informa Healthcare, pp. 301-348.

DHAHC (2004) Environmental health risk assessment guidelines for assessing human health risks from environmental hazards. Canberra, Australian Government, Department of Health and Ageing, 227 pp. http://www.health.gov.au/internet/main/publishing.nsf/Content/ohpehra-2004.htm).

Dourson ML, Felter SP, Robinson D (1996) Evolution of science-based uncertainty factors in noncancer risk assessment. Regulatory Toxicology and Pharmacology, 24:108-120.

ECETOC (2003) Derivation of assessment factors for human health risk assessment. Brussels, European Centre for Ecotoxicology and Toxicology of Chemicals (Technical Report No. 86).

Environment Canada, Health Canada (2002) Priority substances list assessment report. 2Butoxyethanol. Ottawa, Ontario, Government of Canada http://www.hc-sc.gc.ca/ewhsemt/alt formats/hecs-sesc/pdf/pubs/contaminants/ps12-1sp2/2_butoxyethanol/ 2 butoxyethanol-eng.pdf. 
Farrar D, Allen B, Crump K, Shipp A (1989) Evaluation of uncertainty in input parameters to pharmacokinetic models and the resulting uncertainties in output. Toxicology Letters, 49:371-385.

Fiserova-Bergerova V (1983) Modeling of inhalation exposure to vapors: uptake distribution and elimination. Vol. 2. Boca Raton, FL, CRC Press, pp. 108-130.

Gehring PJ, Watanabe PG, Park CN (1978) Resolution of dose-response toxicity data for chemicals requiring metabolic activation: example - vinyl chloride. Toxicology and Applied Pharmacology, 44:581-591.

Gelman A, Bois F, Jiang J (1996) Physiological pharmacokinetic analysis using population modeling and informative prior distributions. Journal of the American Statistical Association, 91(436):1400-1412.

Gentry PR, Clewell HJ, Andersen ME (2004) Good modeling practices for pharmacokinetic models in chemical risk assessment. Unpublished contract report submitted to Health Canada, Ottawa, Ontario.

Gerlowski LE, Jain RK (1983) Physiologically based pharmacokinetic modeling: principles and applications. Journal of Pharmaceutical Science, 72:1103-1127.

Gerrity TR, Henry CJ (1990) Principles of route-to-route extrapolation for risk assessment. New York, NY, Elsevier, pp. 1-12.

Gueorguieva I, Aarons L, Rowland M (2006a) Diazepam pharmacokinetics from preclinical to phase I using a Bayesian population physiologically based pharmacokinetic model with informative prior distributions in WinBUGS. Journal of Pharmacokinetics and Pharmacodynamics, 33(5):571-594.

Gueorguieva I, Nestorov IA, Rowland M (2006b) Reducing whole body physiologically based pharmacokinetic models using global sensitivity analysis: diazepam case study. Journal of Pharmacokinetics and Pharmacodynamics, 33(1):1-27.

Gundert-Remy U, Sonich-Mullin C (2002) The use of toxicokinetic and toxicodynamic data in risk assessment: an international perspective. Science of the Total Environment, 288:3-11.

Haber LT, Maier A, Gentry PR, Clewell HJ, Dourson ML (2002) Genetic polymorphisms in assessing interindividual variability in delivered dose. Regulatory Toxicology and Pharmacology, 35:177-197.

Hack CE, Chiu WA, Zhao QJ, Clewell HJ (2006) Bayesian population analysis of a harmonized physiologically based pharmacokinetic model of trichloroethylene and its metabolites. Regulatory Toxicology and Pharmacology, 46(1):63-83.

Hattis D, White P, Koch P (1993) Uncertainties in pharmacokinetic modeling for perchloroethylene: II. Comparison of model predictions with data for a variety of different parameters. Risk Analysis, 13:599-610. 
Health Canada (1994) Human health risk assessment for priority substances. Ottawa, Ontario, Health Canada, Environmental Health Directorate http://www.hc-sc.gc.ca/ewhsemt/alt_formats/hecs-sesc/pdf/pubs/contaminants/approach/approach-eng.pdf).

Himmelstein KJ, Lutz RJ (1979) A review of the application of physiologically based pharmacokinetic modeling. Journal of Pharmacokinetics and Biopharmaceutics, 7:127-145.

ICRP (1975) Report of the Task Group on Reference Man. Prepared by the International Commission on Radiological Protection. New York, NY, Pergamon Press.

IGHRC (2006) Guidelines on route-to-route extrapolation of toxicity data when assessing health risks of chemicals. Bedfordshire, Cranfield University, Institute of Environment and Health, Interdepartmental Group on Health Risks from Chemicals http://ieh.cranfield.ac.uk ighrc/cr12[1].pdf).

Iman RL, Helton JC (1988) An investigation of uncertainty and sensitivity analysis techniques for computer models. Risk Analysis, 8:71-90.

IPCS (1994) Assessing human health risks of chemicals: derivation of guidance values for health-based exposure limits. Geneva, World Health Organization, International Programme on Chemical Safety (Environmental Health Criteria 170; http://www.inchem.org/documents/ ehc/ehc/ehc170.htm.

IPCS (1999) Principles for the assessment of risks to human health from exposure to chemicals. Geneva, World Health Organization, International Programme on Chemical Safety (Environmental Health Criteria 210; http://www.inchem.org/documents/ehc/ehc) ehc210.htm).

IPCS (2004) IPCS risk assessment terminology. Geneva, World Health Organization, International Programme on Chemical Safety (Harmonization Project Document No. 1; http://www.who.int/ipcs/methods/harmonization/areas/ipcsterminologyparts land2.pdf).

IPCS (2005a) Chemical-specific adjustment factors for interspecies differences and human variability: guidance document for use of data in dose/concentration-response assessment. Geneva, World Health Organization, International Programme on Chemical Safety (Harmonization Project Document No. 2; http://whqlibdoc.who.int/publications/2005/ 9241546786_eng.pdf).

IPCS (2005b) Principles of characterizing and applying human exposure models. Geneva, World Health Organization, International Programme on Chemical Safety (Harmonization Project Document No. 3; http://whqlibdoc.who.int/publications/2005/9241563117 eng.pdf).

IPCS (2008) Part 1. Guidance document on characterizing and communicating uncertainty in exposure assessment. In: Uncertainty and data quality in exposure assessment. Geneva, World Health Organization, International Programme on Chemical Safety (Harmonization Project Document No. 6; http://www.who.int/ipcs/publications/methods/harmonization/ exposure_assessment.pdt). 
IPCS (2009) Principles and methods for the risk assessment of chemicals in food. Geneva, World Health Organization, International Programme on Chemical Safety (Environmental Health Criteria 240; http://www.who.int/ipcs/food/principles/en/index1.html).

Iyengar S, Rao MS (1983) Statistical techniques in modeling of complex systems: single versus multiresponse models. IEEE Transactions on Systems, Man and Cybernetics, 13:175189.

Jacobs A (2007) Drug development and the use of pharmacokinetics/toxicokinetics in selecting the first dose of systemically administered drugs in humans - a nonclinical perspective. In: Lipscomb JC, Ohanian EV, eds. Toxicokinetics and risk assessment. New York, NY, Informa Healthcare, pp. 285-300.

Jarabek AM (1994) Inhalation RfC methodology: dosimetric adjustments and dose-response estimation of non-cancer toxicity in the upper respiratory tract. Inhalation Toxicology, 6:301325 .

Jarabek AM (1995) The application of dosimetry models to identify key processes and parameters for default dose-response assessment approaches. Toxicology Letters, 79:171184.

Johanson G, Johnsson S (1991) Gas chromatographic determination of butoxyacetic acid in human blood after exposure to 2-butoxyethanol. Archives of Toxicology, 65(5):433-435.

Jonsson F, Johanson G (2001) A Bayesian analysis of the influence of GSTT1 polymorphism on the cancer risk estimate for dichloromethane. Toxicology and Applied Pharmacology, 174(2):99-112.

Jonsson F, Johanson G (2002) Physiologically based modeling of the inhalation kinetics of styrene in humans using a Bayesian population approach. Toxicology and Applied Pharmacology, 179:35-49.

Kalvass JC, Maurer TS (2002) Influence of nonspecific brain and plasma binding on CNS exposure: implications for rational drug discovery. Biopharmaceutics \& Drug Disposition, 23(8):327-338.

Kirman CR, Sweeney LM, Meek ME, Gargas ML (2003) Assessing the dose-dependency of allometric scaling performance using physiologically based pharmacokinetic modeling. Regulatory Toxicology and Pharmacology, 38:345-367.

Kohn MC (1995) Achieving credibility in risk assessment models. Toxicology Letters, 79:107-114.

Konietzka R, Dieter HH, Schneider K (2008) Toxicological evaluations: extrapolation beats uncertainty. Journal of Toxicology and Environmental Health, Part B, 11:609-611.

Krewski D, Wang Y, Bartlett S, Krishnan K (1995) Uncertainty, variability, and sensitivity analysis in physiological pharmacokinetic models. Journal of Biopharmaceutical Statistics, $5: 245-271$. 
Krishnan K, Andersen ME (2007) Physiologically-based pharmacokinetic and toxicokinetic modeling. In: Hayes AW, ed. Principles and methods in toxicology. New York, NY, Taylor and Francis, pp. 232-291.

Krishnan K, Carrier R (2008) Exposure source and multiroute exposure considerations for risk assessment of drinking water contaminants. In: Howd RA, Fan AM, eds. Risk assessment for chemicals in drinking water. Hoboken, NJ, John Wiley \& Sons, pp. 67-90.

Krishnan K, Haddad S, Beliveau M, Tardif R (2002) Physiological modeling and extrapolation of pharmacokinetic interactions from binary to more complex chemical mixtures. Environmental Health Perspectives, 110(6):989-994.

Lee KM, Dill JA, Chou BJ, Roycroft JH (1998) Physiologically based pharmacokinetic model for chronic inhalation of 2-butoxyethanol. Toxicology and Applied Pharmacology, 153(2):211-226.

Leijnse A, Hassanizadeh SM (1994) Model definition and model validation. Advances in Water Resources, 17(3):197-200.

Lipscomb JC, Ohanian EV, eds (2007) Toxicokinetics and risk assessment. New York, NY, Informa Healthcare, $361 \mathrm{pp}$.

Lipscomb JC, Poet TS (2008) In vitro measurements of metabolism for application in pharmacokinetic modeling. Pharmacology \& Therapeutics, 118:82-103.

Lipscomb JC, Fisher JW, Confer PD, Byczkowski JZ (1998) In vitro to in vivo extrapolation for trichloroethylene metabolism in humans. Toxicology and Applied Pharmacology, $152: 376-387$.

Loizou G, Spendiff M, Barton HA, Bessems J, Bois FY, d'Yvoire MB, Buist H, Clewell HJ, Meek B, Gundert-Remy U, Goerlitz G, Schmitt W (2008) Development of good modeling practice for physiologically based pharmacokinetic models for use in risk assessment: the first steps. Regulatory Toxicology and Pharmacology, 50(3):400-411.

Marcus AH, Elias R (1998) Some useful statistical methods for model validation. Environmental Health Perspectives, 106(6):1541-1550.

Meek ME, Renwick A, Sonich-Mullin C (2003a) Practical application of kinetic data in risk assessment — an IPCS initiative. Toxicology Letters, 138:151-160.

Meek ME, Bucher JR, Cohen SM, Dellarco V, Hill RN, Lehman-McKeeman LD, Longfellow DG, Pastoor T, Seed J, Patton DE (2003b) A framework for human relevance analysis of information on carcinogenic modes of action. Critical Reviews in Toxicology, 33:591-653.

Naumann BD, Silverman KC, Dixit R, Faria EC, Sargent EV (2001) Case studies of categorical data-derived adjustment factors. Human and Ecological Risk Assessment, 7(1):61-105. 
Nestorov IA (2001) Modelling and simulation of variability and uncertainty in toxicokinetics and pharmacokinetics. Toxicology Letters, 120:411-420.

NRC (1983) Risk assessment in the federal government: managing the process. Prepared by the Committee on the Institutional Means for Assessment of Risks to Public Health, National Research Council. Washington, DC, National Academy Press (http://www.nap.edu openbook.php?isbn=0309033497).

NRC (1987) Pharmacokinetics in risk assessment: drinking water and health. Vol. 8. Prepared by the Safe Drinking Water Committee, National Research Council. Washington, DC, National Academy Press.

Oreskes N (1998) Evaluation (not validation) of quantitative models. Environmental Health Perspectives, 106(6):1453-1460.

Pauluhn J (2003) Issues of dosimetry in inhalation toxicity. Toxicology Letters, 140:229-238.

Price PS, Keenan RE, Schwab B (1999) Defining the interindividual (intraspecies) uncertainty factor. Human and Ecological Risk Assessment, 5(5):1023-1035.

Price PS, Conolly RB, Chaisson CF, Gross EA, Young JS, Mathis ET, Tedder DR (2003) Modeling interindividual variation in physiological factors used in PBPK models of humans. Critical Reviews in Toxicology, 33:469-503.

Ramsey JC, Andersen ME (1984) A physiological model for the inhalation pharmacokinetics of inhaled styrene monomer in rats and humans. Toxicology and Applied Pharmacology, 73:159-175.

Reddy MB, Yang RSH, Clewell HJ, Andersen ME, eds (2005) Physiologically based pharmacokinetic modeling: science and application. Hoboken, NJ, John Wiley \& Sons, 420 pp.

Renwick AG (1993) Data safety factors for the evaluation of food additives and environmental contaminants. Food Additives and Contaminants, 10:275-305.

Renwick AG (1999) Subdivision of uncertainty factors to allow for toxicokinetics and toxicodynamics. Human and Ecological Risk Assessment, 5(5):1035-1050.

Renwick AG, Dorne JL, Walton K (2001) Pathway-related factors: the potential for human data to improve the scientific basis of risk. Human and Ecological Risk Assessment, $7(1): 165-180$.

Rescigno A, Beck J (1987) The use and abuse of models. Journal of Pharmacokinetics and Biopharmaceutics, 15(3):327-344.

Rideout VC (1991) Mathematical and computer modeling of physiological systems. New York, NY, Prentice-Hall, 272 pp. 
Ritter L, Totman C, Krishnan K, Carrier R, Vezina A, Morisset V (2007) Deriving uncertainty factors for threshold chemical contaminants in drinking water. Journal of Toxicology and Environmental Health, Part B, 10:527-557.

Rodgers T, Rowland M (2007) Mechanistic approaches to volume of distribution predictions: understanding the processes. Pharmaceutical Research, 24(5):918-933.

Rowland M (1985) Physiologic pharmacokinetic models and interanimal species scaling. Pharmacology and Therapeutics, 29:49-68.

Schmitt W (2008) General approach for the calculation of tissue to plasma partition coefficients. Toxicology In Vitro, 22(2):457-467.

Slikker W, Andersen ME, Bogdanffy MS, Bus JS, Cohen SD, Conolly RB, David RM, Doerrer NG, Dorman DC, Gaylor DW, Hattis D, Rogers JM, Setzer WR, Swenberg JA, Wallace K (2004) Dose-dependent transitions in mechanisms of toxicity. Toxicology and Applied Pharmacology, 201(3):203-225.

Sonich-Mullin C, Fielder R, Wiltse J, Baetcke K, Dempsey J, Fenner-Crisp P, Grant D, Hartley M, Knaap A, Kroese D, Mangelsdorf I, Meek E, Rice JM, Younes M (2001) IPCS conceptual framework for evaluating a mode of action for chemical carcinogenesis. Regulatory Toxicology and Pharmacology, 34:46-52.

Tan YM, Liao KH, Clewell HJ III (2007) Reverse dosimetry: interpreting trihalomethanes biomonitoring data using physiologically based pharmacokinetic modeling. Journal of Exposure Science and Environmental Epidemiology, 17:591-603.

Teorell T (1937a) Kinetics of distribution of substances administered to the body. I. The extravascular modes of administration. Archives Internationales de Pharmacodynamie et de Thérapie, 57:205-225.

Teorell T (1937b) Kinetics of distribution of substances administered to the body. II. The intravascular modes of administration. Archives Internationales de Pharmacodynamie et de Thérapie, 57:226-240.

Thompson CM, Sonawane B, Barton HA, DeWoskin RS, Lipscomb JC, Schlosser P, Chiu WA, Krishnan K (2008) Approaches for applications of physiologically based pharmacokinetic models in risk assessment. Journal of Toxicology and Environmental Health, Part B, 11(7):519-547.

Thompson CM, Johns DO, Sonawane B, Barton HA, Hattis D, Tardif R, Krishnan K (2009) Database for physiologically based pharmacokinetic (PBPK) modeling: physiological data for healthy and health-impaired elderly. Journal of Toxicology and Environmental Health, Part B, 12:1-24.

USEPA (2000) Toxicological review of vinyl chloride (CASRN 75-01-4) in support of summary information on the Integrated Risk Information System (IRIS). Washington, DC, United States Environmental Protection Agency (EPA/635/R-00/004). 
USEPA (2006) Approaches for the application of physiologically based pharmacokinetic (PBPK) models and supporting data in risk assessment (final report). Washington, DC, United States Environmental Protection Agency (EPA/600/R-05/043A).

USEPA (2010) Toxicological review of ethylene glycol monobutyl ether (EGBE) (CASRN 111-76-2) in support of summary information on the Integrated Risk Information System (IRIS). Washington, DC, United States Environmental Protection Agency (EPA/635/R08/006F).

van de Sandt JJM, Bellarco M, van Hemmen JJ (2007) From dermal exposure to internal dose. Journal of Exposure Science and Environmental Epidemiology, 17(Suppl. 1):S38-47.

Veerkamp W, Wolff C (1996) Fate and exposure models in relation to risk assessment. Environmental Science and Pollution Research, 3(2):91-95.

Vermeire T, Stevenson H, Pieters MN, Rennen M, Slob W, Hakkert BC (1999) Assessment factors for human health risk assessment: a discussion paper. Critical Reviews in Toxicology, 29(5):439-490.

Verner MA, Ayotte P, Muckle G, Charbonneau M, Haddad S (2009) A physiologically based pharmacokinetic model for the assessment of infant exposure to persistent organic pollutants in epidemiologic studies. Environmental Health Perspectives, 117(3):481-487.

Voisin EM, Ruthsatz M, Collins JM, Hoyles PC (1990) Extrapolation of animal toxicity to humans: interspecies comparisons in drug development. Regulatory Toxicology and Pharmacology, 12:107-116.

Walton K, Dorne JL, Renwick AG (2001a) Uncertainty factors for chemical risk assessment: interspecies differences in glucuronidation. Food and Chemical Toxicology, 39:1175-1190.

Walton K, Dorne JL, Renwick AG (2001b) Uncertainty factors for chemical risk assessment: interspecies differences in the in vivo pharmacokinetics and metabolism of human CYP1A2 substrates. Food and Chemical Toxicology, 39:667-680.

Walton K, Dorne JL, Renwick AG (2001c) Categorical default factors for interspecies differences in the major routes of xenobiotic elimination. Human and Ecological Risk Assessment, 7:181-201.

Walton K, Dorne JL, Renwick AG (2004) Species-specific uncertainty factors for compounds eliminated principally by renal excretion in humans. Food and Chemical Toxicology, 42(2):261-274.

Welsch F, Blumenthal GM, Conolly RB (1995) Physiologically based pharmacokinetic models applicable to organogenesis: extrapolation between species and potential use in prenatal toxicity risk assessments. Toxicology Letters, 82-83:539-547.

Willmann S, Höhn K, Edginton A, Sevestre M, Solodenko J, Weiss W, Lippert J, Schmitt W (2007) Development of a physiology-based whole-body population model for assessing the influence of individual variability on the pharmacokinetics of drugs. Journal of Pharmacokinetics and Pharmacodynamics, 34:401-431. 
Yoon M, Barton, HA (2008) Predicting maternal rat and pup exposures: how different are they? Toxicological Sciences, 102(1):15-32.

Younes M, Sonich-Mullin C, Meek ME (1998) Risk: assessment and management. In: Herzstein JA, Bunn WB, Fleming LE, eds. International occupational and environmental medicine. St Louis, MO, Mosby, pp. 62-74. 


\section{ANNEX 1: GLOSSARY OF TERMS'}

Absorbed dose: The amount of chemical that, after contact with the exchange boundary (skin, lungs, gut), actually penetrates the exchange boundary and enters the circulatory system. The amount may be the same as or less than the applied dose.

Adjustment factor: See Chemical-specific adjustment factor.

Adverse effect: The change in morphology, physiology, growth, development or lifespan of an organism that results in impairment of functional capacity, impairment of capacity to compensate for additional stress or increase in susceptibility to the harmful effects of other environmental influences.

Algorithm: A fixed, step-by-step mathematical procedure.

Area under the curve (AUC): Area under the concentration versus time curve. The AUC is a summary measure that integrates serial assessments of internal dose over the duration of a study.

Assessment factor: See Uncertainty factor.

Benchmark concentration (BMC): The concentration calculated to be associated with a given incidence (e.g. $5 \%$ or $10 \%$ incidence) of effect estimated from all toxicity data on that effect within that study. BMCL is the statistical lower confidence limit of the BMC.

Benchmark dose (BMD): The dose calculated to be associated with a given incidence (e.g. $5 \%$ or $10 \%$ incidence) of effect estimated from all toxicity data on that effect within that study. BMDL is the statistical lower confidence limit of the BMD.

Biologically effective dose: The amount of the chemical available for interaction with any particular organ, cell or macromolecular target.

Chemical-specific adjustment factor (CSAF): A factor based on quantitative chemicalspecific toxicokinetic or toxicodynamic data, which replaces the default uncertainty factor.

Critical effect: The first adverse effect, or its known precursor, that occurs in the dose/concentration scale.

Default value: Pragmatic, fixed or standard value used in the absence of relevant data.

Delivered dose: The amount of a substance available for biologically significant interactions in the target organ.

Diffusion-limited uptake: Occurs when the diffusion of a chemical (typically high molecular weight chemicals and those with significant protein binding) across the membrane is the rate-limiting process of tissue uptake.

\footnotetext{
${ }^{1}$ Based on Carson et al. (1983), Oreskes (1998), DHAHC (2004), IPCS (2004, 2005a) and USEPA (2006).
} 
Dose: A stated quantity or concentration of a substance to which an organism is exposed over a continuous or intermittent duration of exposure. It is most commonly expressed as the amount of test substance per unit weight of test animal (e.g. $\mathrm{mg} / \mathrm{kg}$ body weight).

Dose metric: The target tissue dose that is closely related to ensuing adverse responses. Dose metrics used for risk assessment applications should reflect the biologically active form of the chemical, its level, duration of internal exposure as well as intensity.

Dose-response assessment: The process of determining the relationship between the magnitude of administered, applied or internal doses and biological responses. Response can be expressed as measured or observed incidence or change in level of response, per cent response in groups of subjects (or population) or the probability of occurrence or change in level of response within a population.

Exposure: Contact between an agent and a target.

Exposure dose: Amount of an agent presented to an absorption barrier and available for absorption-i.e. the amount ingested, inhaled or applied to the skin. This amount may be the same as or greater than the absorbed dose. Also referred to as applied dose or potential dose.

First-order process: A linear process whose output is strictly proportional to the dose or concentration.

First-pass effects: Metabolism that occurs before a compound can enter the general circulation. For example, an orally administered compound may undergo metabolism in the intestines and/or liver prior to systemic distribution.

Fitting: Process of optimizing model output to experimental data for the estimation of parameters adequately identifiable from the data.

Flow-limited uptake: Occurs when the chemical diffuses readily between blood and tissue compartments and exchange is limited primarily by blood flow.

Human-equivalent concentration or dose: The human concentration (for inhalation exposure) or dose (for oral exposure) of an agent that is believed to induce the same magnitude of toxic effect as the exposure concentration or dose in experimental animal species. This adjustment may incorporate toxicokinetic information on the particular agent, if available, or use a default procedure.

Internal dose: A general term denoting the amount absorbed or the concentration of a chemical (or its metabolites and adducts) in biological matrices.

Lowest-observed-adverse-effect concentration (LOAEC): The lowest concentration of a substance, found by experiment or observation, that causes an adverse alteration of morphology, functional capacity, growth, development or lifespan of the target organisms distinguishable from normal (control) organisms of the same species and strain under the same defined conditions of exposure.

Lowest-observed-adverse-effect level (LOAEL): The lowest amount of a substance, found by experiment or observation, that causes an adverse alteration of morphology, functional 
capacity, growth, development or lifespan of the target organisms distinguishable from normal (control) organisms of the same species and strain under the same defined conditions of exposure.

Markov chain Monte Carlo: A simulation approach that considers a model's parameters as random variables with a probability distribution for describing each parameter. The distribution based only on prior information and assumptions is called the prior distribution. Analysis of new data yields a posterior distribution of parameters that reconciles the prior information and assumptions with the new data.

Mechanism of action: A detailed description of the precise chain of events from the molecular level to gross macroscopic or histopathological toxicity.

Mode of action (MOA): A series of key events that may lead to induction of the relevant end-point of toxicity for which the weight of evidence supports plausibility.

Model evaluation: Refers to the process of establishing confidence in a model on the basis of scientific principles, quality of input parameters and ability to reproduce independent empirical data. In the context of PBPK models, evaluation is purpose specific and focuses on the following aspects: biological basis of the model, model simulations of data and reliability of dose metric predictions.

Monte Carlo simulation: Repeated random sampling from the distribution of input parameters to derive a distribution of output in the population. Monte Carlo simulation with PBPK models can provide population distributions of dose metric of relevance to risk assessment.

Non-threshold toxicant: A chemical for which there is no dose or exposure concentration below which the critical effect will not be observed or expected to occur.

No-observed-adverse-effect concentration (NOAEC): The highest concentration of a substance, found by experiment or observation, that causes no detectable adverse alteration of morphology, functional capacity, growth, development or lifespan of the target organisms under defined conditions of exposure.

No-observed-adverse-effect level (NOAEL): The highest amount of a substance, found by experiment or observation, that causes no detectable adverse alteration of morphology, functional capacity, growth, development or lifespan of the target organisms under defined conditions of exposure.

Perfusion-limited uptake: See Flow-limited uptake.

Pharmacodynamic (PD) models: Mathematical descriptions simulating the relationship between a biologically effective dose and the occurrence of a tissue response over time.

Pharmacodynamics (PD): See Toxicodynamics.

Pharmacokinetic (PK) models: Mathematical descriptions simulating the relationship between external exposure level and chemical concentration in biological matrices over time. 
PK models take into account absorption, distribution, metabolism and elimination of the administered chemical and its metabolites.

Pharmacokinetics (PK): See Toxicokinetics.

Physiologically based pharmacokinetic (PBPK) model: A model that estimates the dose to target tissue by taking into account the rate of absorption into the body, distribution and storage in tissues, metabolism and excretion on the basis of interplay among critical physiological, physicochemical and biochemical determinants.

Point of departure (POD): The dose-response point that marks the beginning of a low-dose extrapolation. This point can be the lower bound on dose for an estimated incidence or a change in response level from a dose-response model (benchmark dose or concentration) or a no-observed-adverse-effect level or lowest-observed-adverse-effect level for an observed incidence or change in level or response.

Reliability: In the context of PBPK modelling in risk assessment, refers to the trustworthiness of the model for its prediction of dose metrics. The reliability is assessed on the basis of how well the model has been tested against real data and whether adequate sensitivity and uncertainty analyses have been conducted to support the model's ability to provide prediction of dose metrics.

Sensitivity analysis: Quantitative evaluation of how input parameters influence the model output (e.g. dose metrics).

Simulation: System behaviour (e.g. blood kinetic profile in exposed organism) predicted by solving the differential and algebraic equations constituting a model.

Steady state: A variable is said to have attained steady state when its value stays constant in a given interval of time (i.e. when its derivative is zero).

Target organ (or tissue): The biological organ (or tissue) most adversely affected by exposure to a chemical or physical agent.

Threshold toxicant: A chemical for which the critical effect is observed or expected to occur only above a certain dose or exposure concentration.

Toxicodynamics (TD): The process of interaction of chemical substances with target sites and the subsequent reactions leading to adverse effects. The term has essentially the same meaning as pharmacokinetics, but the latter term is frequently used in reference to pharmaceutical substances.

Toxicokinetics (TK): The process of the uptake of potentially toxic substances by the body, the biotransformation they undergo, the distribution of the substances and their metabolites in the tissues and the elimination of the substances and their metabolites from the body. Both the amounts and the concentrations of the substances and their metabolites are studied. The term has essentially the same meaning as pharmacokinetics, but the latter term is frequently used in reference to pharmaceutical substances. 
Uncertainty: Refers to lack of knowledge. Uncertainty can often be reduced with greater knowledge of the system or by collecting more and better experimental or simulation data.

Uncertainty factor: A product of several single factors by which the no-observed-adverseeffect level or lowest-observed-adverse-effect level of the critical effect is divided to derive a tolerable intake. These factors account for adequacy of the pivotal study, interspecies extrapolation, interindividual variability in humans, adequacy of the overall database and nature of toxicity.

Validation: Process by which the reliability and relevance of a particular approach (or model) is established for a defined purpose.

Variability: Refers to true heterogeneity or diversity. Differences among individuals in a population are referred to as interindividual variability; differences for one individual over time are referred to as intraindividual variability.

Verification: In the context of PBPK modelling, refers to the process of examining the model structure, parameters, units, equations and model codes to ensure accuracy. 


\section{ANNEX 2: FREQUENTLY ASKED QUESTIONS}

Which specific uncertainty factors can be refined or replaced with the simulations of PBPK models?

- $\mathrm{AK}_{\mathrm{AF}}$, i.e. the TK component of the interspecies uncertainty factor (central estimate); and

- $\mathrm{HK}_{\mathrm{AF}}$, i.e. the $\mathrm{TK}$ component of the interindividual uncertainty factor (population variability).

Will the use of a PBPK model always result in a reduction of uncertainty factors compared with the default values?

No. The assessment factors derived on the basis of PBPK model simulations of dose metrics may be equal to, greater than or less than the default values.

What is the relationship between PBPK models and CSAFs?

CSAFs refer to chemical-specific adjustment factors derived on the basis of TK data relevant to the MOA of chemicals. If relevant experimental data useful for deriving CSAFs are not available, PBPK models may be used to simulate the dose metrics required for calculating the kinetic components of $\mathrm{CSAF}$, i.e. $\mathrm{AK}_{\mathrm{AF}}$ and $\mathrm{HK}_{\mathrm{AF}}$.

When PBPK models are not available for the species or exposure route relevant to the critical toxicological study, how do I use the PBPK model in a risk assessment?

It will be of only limited use, particularly for informing about the linearity of the internal dose versus external dose relationship in a given species as well as for exploring the appropriate dose metrics of relevance to the MOA of the chemical.

Will PBPK modelling always suggest non-linearity in a dose-response relationship?

No. If all metabolic and physiological processes in a given species are linear in the dose range of interest, then the dose-response analysis conducted with the PBPK model will indicate no departure from linearity.

Are PBPK models available for all chemicals?

No. There are compilations and web sites that list PBPK models published to date for specific chemicals and species (Corley et al., 2003; Reddy et al., 2005; Krishnan \& Andersen, 2007; http://www.pbpk.org.

What kind of factor is applied to account for remaining uncertainty (i.e. TD differences) once a PBPK model is applied to account for interspecies TK differences?

A default factor of 2.5 is applied to account for remaining uncertainty (IPCS, 2005a). However, chemical-specific information on the interspecies differences in TD may lead to a further reduction or increase of this default value. 
What kind of factor is applied to account for remaining uncertainty (i.e. TD differences) once a PBPK model is applied to account for interindividual TK differences?

A default factor of 3.16 is applied to account for remaining uncertainty (IPCS, 2005a). However, chemical-specific information on the human variability in toxicodynamics may lead to a further reduction or increase of this default value.

Do we always need a PBPK model to account for interspecies or interindividual TK differences in risk assessments?

No. If experimental data on relevant dose metrics are available for the exposure scenarios and species (or population) of interest, then those data can be used to calculate CSAFs.

Would a multicompartmental PBPK model always be superior to a one-compartment model?

No. For example, for chemicals whose concentration versus time profiles are identical throughout the body (e.g. certain hydrophilic chemicals), the use of one-compartment models would be sufficient.

Are all PBPK models useful in quantifying the population variability of dose metric and response?

No. Only those PBPK models that contain information on the variability of input parameters for the population of interest would be useful in providing information on the distribution of dose metrics.

Does the use of PBPK models in risk assessment render the latter process less or more conservative compared with the conventional approach?

It can go either way or end up giving the same results as the conventional approach. In any case, the PBPK models contribute to augment the scientific basis of risk assessments by basing them on internal dose (i.e. more closely related to tissue response) rather than external dose of chemicals.

Do all PBPK models account for physiological and metabolic alterations, if any, to facilitate the prediction of dose metrics during chronic exposures?

No. Not all PBPK models account for dose-related pathophysiological or biochemical changes that may occur during repeated exposure to high doses of chemicals.

If there are multiple PBPK models for a given chemical, how do I proceed?

Choose the model that 1) has the least uncertainty in predictions of the dose metrics of relevance to the MOA and 2) has been calibrated and evaluated for the exposure routes, dose ranges, species and life stage of relevance to the assessment. 
Can the PBPK models provide an estimate of uncertainty factors used to extrapolate from subchronic to chronic exposure and from LOAEL to NOAEL?

No. PBPK models cannot be used in isolation for assessing or suggesting the appropriate magnitude of these factors. Therefore, the toxicological data, dose-response relationship as well as the MOA information would continue to be the critical pieces of information supporting or refuting the default values for these uncertainty factors.

\section{What constitutes adequate "validation" in the case of PBPK models?}

Validation and evaluation of PBPK models are context specific and should be conducted by considering the 1) biological basis of the model structure and parameters, 2) closeness of the model simulations to experimental data and 3) reliability of model predictions of dose metrics relevant to the risk assessment. Supplementary analyses of variability, uncertainty and sensitivity might be important, depending upon the end use and extent of comparison with real-life data.

Are more complex PBPK models better than simpler ones for application in risk assessment?

Not necessarily. In principle, the credibility and usefulness of models should not be equated to model complexity.

\section{What constitutes adequate "simulation" in PBPK modelling?}

In PBPK modelling, simulations that are on average within a factor of 2 of the experimental data have frequently been considered adequate.

Is variability analysis required for all PBPK models intended for risk assessment application?

No. The focus of a variability analysis is to evaluate the range of values that a parameter is expected to have in a population and its impact on the variability of the dose metric. Such an analysis is fundamental to the use of PBPK models in estimating $\mathrm{HK}_{\mathrm{AF}}$ (i.e. TK component of the interindividual uncertainty factor). However, variability analysis is not a prerequisite for a PBPK model intended for use in interspecies, high dose to low dose or route-to-route extrapolations.

What is uncertainty analysis, and is it required for all PBPK models intended for risk assessment application?

Uncertainty analysis evaluates the impact of the lack of precise knowledge of parameter values and model structure on dose metric simulations. It would be essential/beneficial for those PBPK models that do not adequately simulate the experimental PK data or that have been evaluated only with limited data sets. 
What is sensitivity analysis, and is it required for all PBPK models intended for risk assessment application?

In the context of PBPK modelling, sensitivity analysis provides a quantitative evaluation of how parameters in input functions influence the dose metrics or other model output of relevance to risk assessment. Although not usually required for all PBPK models, it is critically useful in identifying the key parameters of the model for which variability or uncertainty should be analysed. 


\section{ANNEX 3: CASE-STUDY ON INTEGRATING PBPK MODELS IN A RISK ASSESSMENT OF A CHEMICAL}

The following sections describe essential information to integrate the application and development of a PBPK model into a health risk assessment of a chemical. A template with simple instructions on how to document a PBPK model (section A) and a case-study example for a "hypothetical" chemical (VC) using the template (section B) are presented.

Section A presents the template with information relevant for description of a PBPK model for application in chemical risk assessment. Four areas are included in the template: 1) background on chemical PK and MOA, 2) characterization and evaluation of the PBPK model, 3) modelling and evaluation of the model-derived dose metrics and 4) PBPK modelling and comparison with the default assessment. The brief descriptions of the sections included here should be read in conjunction with the more complete explanations included in the guidance document and the case-study in section B.

Section B applies the case-study template to the hypothetical chemical VC, including an overview of the observed toxicity (liver angiosarcoma) and evidence for the MOA. The predictive capability of the model and confidence in a predicted dose metric (liver concentration of the metabolite VC-M) are evaluated and compared with the outcome using the default approach.

\section{A. CASE-STUDY TEMPLATE}

\section{A1. Background}

Describe the overall objective of the risk assessment. Specifically, what is the intended goal of the risk assessment (e.g. deriving guidance value for the inhalation route based on a cancer bioassay conducted in rats)? State the issue of PK uncertainty pertaining to the specific objectives of the assessment (e.g. calculate the human-equivalent exposure concentration of a chemical that yields the same level of dose metric that produces a given level of effect in the responding test species).

\section{A1.1 Critical effect}

A summary review of the toxicological database on the chemical should be presented with emphasis on the target organs and tissues and effects. This section should also describe the dose-response information associated with the critical studies being considered for the assessment.

\section{A1.2 Pharmacokinetics}

Describe the pathways and processes involved in the absorption, distribution, metabolism and excretion of the chemical. Include a metabolic scheme showing the different pathways and metabolites (particularly the potential dose metrics) with emphasis on dose dependency, species- and route-specific observations as well as the role of enzyme variants, to the extent they are known. 


\section{A1.3 Mode of action/relevant dose metric}

The analysis of the weight of evidence for MOA hypotheses and the choice of the appropriate dose metric are fundamental steps to development and incorporation of a PBPK model in comprehensive risk assessment. Key events in the hypothesized MOA producing the toxicity should be identified and schematically presented (e.g. Figure A1). It should be clear as to whether the supporting database, based on the weight of evidence, satisfies criteria of doseresponse as well as temporal concordance of key events, strength, consistency, specificity and biological plausibility (i.e. reflective of physiological reality and consistent with current state of knowledge). Relevant evidence from in vitro and in vivo studies relating to the key role of the parent chemical, a specific metabolite or a metabolic pathway in the toxicity in test animals and humans should be presented. Supporting data may include observations from studies with genetically altered mice, depletion of cofactors (e.g. GSH), enzyme induction, inhibition, toxicity of metabolites, etc.

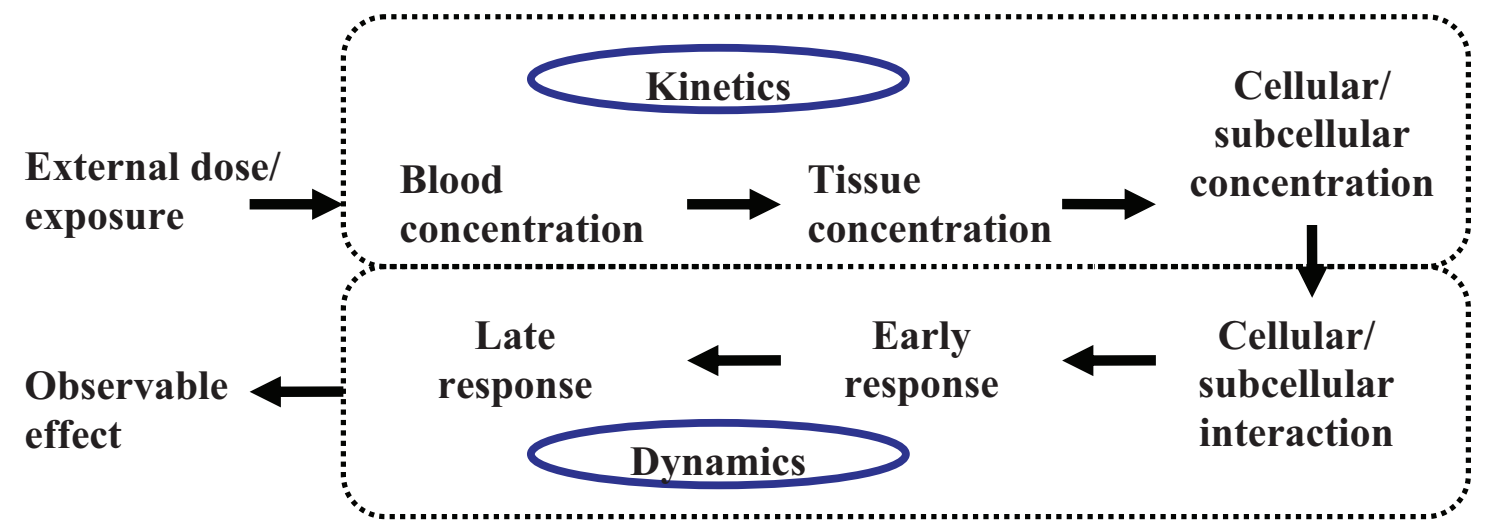

Figure A1: Schematic representation of some key elements of the MOA for the critical doseresponse relationship for a chemical.

Based on the review of the information on MOA for each critical effect, the confidence associated with each of the plausible dose metrics may be summarized, as in Table A1. These degrees of confidence are generally specified based on comparison with the relative degree of support for databases supporting hypothesized dose metrics for other substances. They take into account relative degree of strength (quantity, quality, consistency, specificity and biological plausibility).

Table A1: Confidence in the use of plausible dose metrics for a chemical based on an understanding of MOA for the critical effect (N, none; L, low; M, medium; H, high).

\begin{tabular}{lc}
\hline Dose metric options & Confidence based on MOA \\
\hline Exposure concentration of parent chemical & $\mathrm{N}$ \\
$\ldots$ & $\ldots$ \\
$\ldots$ & $\ldots$ \\
Model-derived dose metric & $? ? ?$ \\
\hline
\end{tabular}

\section{A1.4 Scope for PBPK model application}

This section describes the specific need for the PBPK model and the strategy for use of the modelling results in the risk assessment process. Also, it should identify the specific aspects 
motivating the preferred use of a PBPK model to address the particular risk assessment question or issue.

It is important to identify at this stage the intended application of the model in risk assessment. For example, the PBPK model application might address the following question:

What is the human-equivalent concentration of a risk-specific inhalation exposure in rodents, on the basis of an equivalent dose metric at the target tissue?

It should then be clear as to how the interspecies comparisons are going to be made (e.g. based on measures of central tendency, such as means or medians). Also, depending on the basis for the PBPK model, it should be clarified as to whether a particular model and dose metric together address the interspecies differences in PK only, or PK as well as a portion of PD. This is critically important, for example, as a basis for appropriate application of factors to address the remaining elements of uncertainty for CSAFs.

\section{A2. PBPK model: characterization and evaluation}

\section{A2.1 Model capability and selection}

Given the nature of the risk assessment (e.g. cancer versus non-cancer, inhalation versus oral), the PBPK models selected should be able to simulate potential dose metrics in the needed species, sex, life stage and exposure route (e.g. adult male rats and adult humans exposed by inhalation). If multiple models were developed or available, the rationale for choosing one model over the other should be described. Also, it is essential to describe whether the chosen model is capable of simulating all or only some of the candidate dose metrics identified in Table A1.

\section{A2.2 Model structure and biological characterization}

Describe the model in terms of physiological compartments and biological processes. Specifically, what is the rationale for the choice of specific physiological compartments? Is the structure similar to that of models established for other chemicals or chemical classes? Include a schematic representation of the model that also clearly indicates the extent to which the metabolic scheme is integrated within the model (e.g. Figure A2). The routes of absorption, distribution, including protein binding as well as diffusion- or perfusion-limited uptake, and elimination processes included in the model should be described with respect to the biological basis and mechanisms consistent with information presented in section A1.2.

\section{A2.3 Parameter estimation and analysis}

Describe the sources from which the parameter values were obtained. Were model parameters such as partition coefficients and metabolism constants obtained on the basis of iterative fitting to in vivo data (e.g. plasma time course, total metabolism by gas uptake, GSH depletion, urinary metabolite levels), in vitro data or in silico methods? Describe and justify the use of the in silico method (in terms of application domain), allometric scaling and in vitro-in vivo scaling of parameters, if applicable. When the parameter is not directly available in the cited reference source, but calculated from data reported in that source, it is essential to indicate how the specific values were obtained for use in the model. 


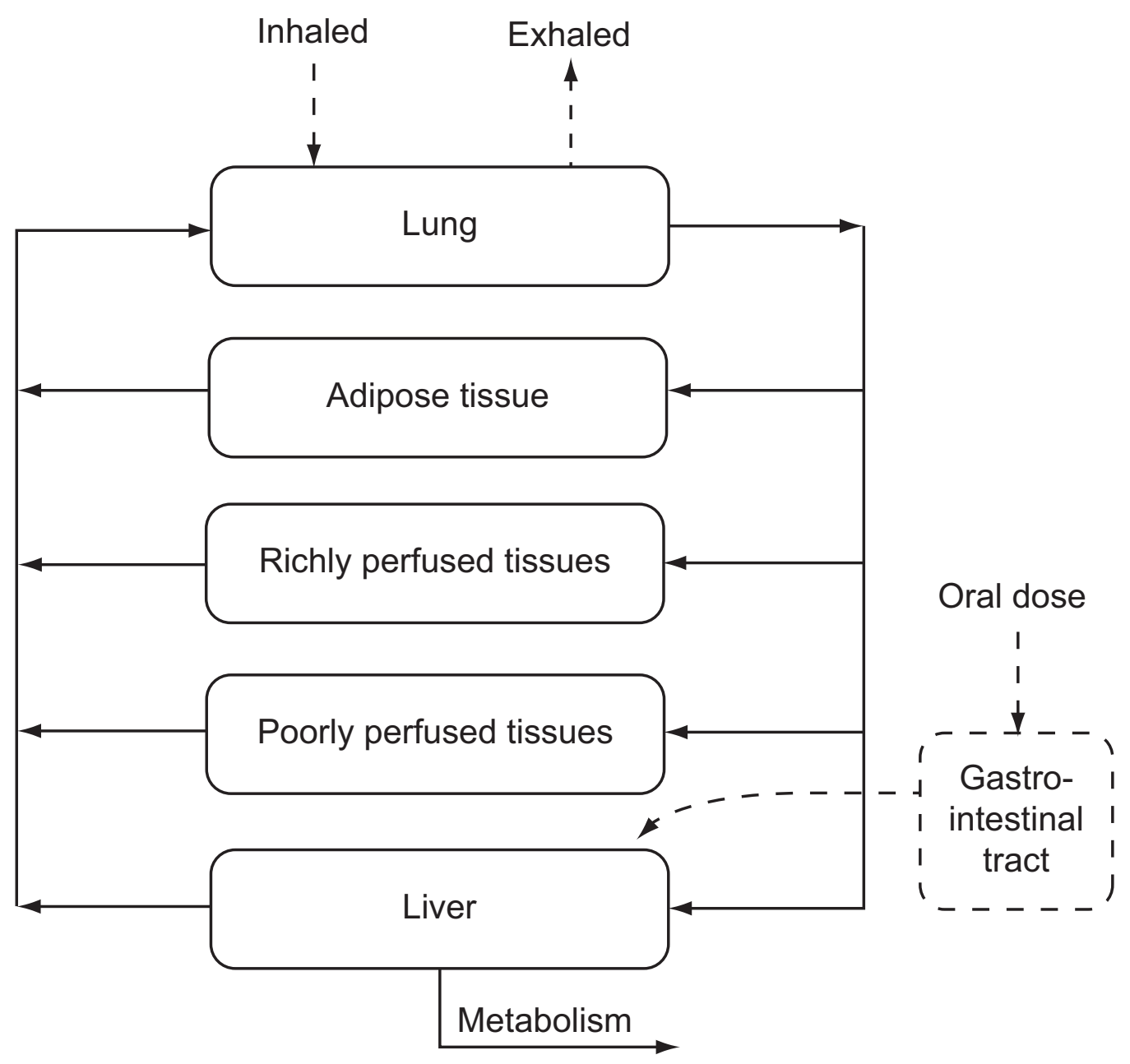

Figure A2: Structure of the PBPK model for a volatile organic chemical.

Key model parameters having significant influence on the dose metric predictions (model outcome) for both test animals and humans should be provided here, preferably in tabular form. Where possible, the complete list of all model parameters should be appended to this document along with their values and sources. Appendix B1 of the VC example presents the key parameters that are most influential in determining the outcome of the model. Even though all parameters may not be critical to the dose metric prediction in humans and test animals, some are likely to be relevant to model simulation and parameter estimation in other studies or for other purposes (e.g. exposure routes not in the current risk assessment).

\section{A2.4 Purpose-specific model evaluation}

Describe the evaluation of the model, focusing on the level of confidence in its structure and parameters as well as its ability to adequately predict the dose metrics for the intended risk assessment application. The reliability of the PBPK model for the specific purpose for which it is intended-for example, conducting interspecies extrapolation of $\mathrm{PK}$ - should be described.

Provide a summary list of experimental data/studies that were compared with the simulations of the PBPK model. Based on simulation outputs, indicate whether the model reproduces the shape of the time course PK data in experimental animals and humans. Does the model 
consistently reproduce the general trend of the data (i.e. peaks, bumps and valleys, saturation of metabolism) or only portions of one or more data sets? How close are the model simulations to the experimental data (e.g. are they within a factor of 2 on average?), given that the experimental data in reality constitute only one sample of the hypothetically plausible range of values?

The level of confidence in the PBPK model for its predictions of dose metrics intended for the risk assessment purpose (e.g. interspecies extrapolation of PK) should be established on the basis of the following considerations (see section 3.7):

- Biological basis: Do the model structure and parameters have a reasonable biological basis?

- Model simulations of data: How well does the PBPK model reproduce the chemicalspecific TK data under various experimental or exposure conditions?

- Reliability (model testing, uncertainty and sensitivity): How reliable is the PBPK model with regard to its predictions of dose metrics relevant to risk assessment?

Describe the reliability of the model predictions of dose metrics for the risk assessment, where feasible, based on the level of sensitivity of the predictions to the model parameters and the level of uncertainty of the parameter values (study origins, estimated from data or experimental measurements). If the highly sensitive parameters are also the ones that are highly uncertain, then the reliability of the model for risk assessment applications would be questionable. The results may be presented in summary form, as illustrated in Figure A3. The quantitative values describing low, medium and high uncertainty or sensitivity should be assigned on a case-by-case basis, depending upon, among other things, the expected distribution of the variables considered. Although the scaling of this analysis is subjectively defined, the qualitative overview of sensitivity and uncertainty conveys the expected behaviour of the model and parameter confidence by focusing on:

- for the human risk assessment conditions (e.g. exposure pathways, relevant exposure conditions such as acute or chronic), which parameters most strongly influence the dose metric; and

- for the study or studies from which the critical end-points are derived (i.e. toxicity, epidemiological, clinical studies), which are the model parameters to which the dose metric predictions are most sensitive.

\section{A2.5 Model documentation}

How was the model implemented? Which simulation languages were used for model implementation? Is the model code available from the corresponding author? Have the authors provided the numerical values of all model parameters as well as distributions of parameters used for variability/uncertainty analysis, if applicable?

\section{A2.6 Model peer review}

Were the PBPK models used in the assessment published in the peer-reviewed literature? Was there any further independent review of these models and codes? Did any regulatory agency evaluate or use these models for specific risk assessment applications? What was the nature of these peer reviews? 


\begin{tabular}{|c|c|c|c|c|}
\hline & & \multicolumn{3}{|c|}{ UNCERTAINTY } \\
\hline & & High & Medium & Low \\
\hline \multirow{3}{*}{ 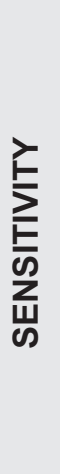 } & 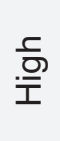 & & Parameter 1 & $\begin{array}{l}\text { Parameter } 3 \\
\text { Parameter } 4 \\
\text { Parameter } 7\end{array}$ \\
\hline & $\begin{array}{l}\frac{E}{3} \\
\frac{.}{\overline{0}} \\
\frac{0}{2}\end{array}$ & & Parameter 2 & Parameter 10 \\
\hline & ב⿱ & & & $\begin{array}{l}\text { Parameter } 12 \\
\text { Parameter } 13\end{array}$ \\
\hline
\end{tabular}

Figure A3: A sample summary table of the output of the uncertainty and sensitivity analyses for the PBPK model of a chemical. Sensitivity and uncertainty analysis results are presented as high, medium or low.

\section{A3. PBPK modelling and evaluation of dose metrics}

Present the dose metrics computed with the PBPK model for the POD for a particular critical end-point (e.g. NOAEL) or the dose metrics associated with each of the treatment groups (e.g. BMD analysis). Describe the relationship between the various dose metrics and responses observed in critical studies. Which alternative dose metrics were evaluated, and what was the reason for choosing the particular dose over the others?

Evaluate the confidence in the choice of the dose metric based on the reliability of model predictions, consideration of the dose-response information for the end-point of concern in the key studies as well as any other available relevant studies acknowledging differences in exposure scenario, route and species. These observations can be summarized as in Table A2 to provide comparisons of the level of confidence in alternative dose metrics, on the basis of both MOA and PBPK modelling. The summary of levels of confidence in the various dose metrics allows the assessor to choose the appropriate dose metrics for the assessment that would be consistent with the nature of the assessment, understanding fully the trade-offs and level of effort required.

Table A2: Confidence in the use of plausible dose metrics for a chemical based on an understanding of MOA for the critical effect and PBPK modelling results (N, none; L, low; $M$, medium; $H$, high).

\begin{tabular}{lcc}
\hline Dose metric options & $\begin{array}{c}\text { Confidence } \\
\text { based on MOA }\end{array}$ & $\begin{array}{c}\text { Confidence in simulation } \\
\text { based on PBPK modelling }\end{array}$ \\
\hline Exposure concentration of parent chemical & $\mathrm{N}$ & $\mathbf{N}$ \\
$\ldots$ & $\ldots$ & $\ldots$ \\
$\ldots$ & $\ldots$ & $\ldots$ \\
Model-derived dose metric & $? ? ?$ & $? ? ?$ \\
\hline
\end{tabular}


This section should also present the results of dose-response modelling based on the dose metrics generated using the PBPK models (in lieu of exposure concentrations or exposure doses), to conduct various extrapolations for deriving the human exposure values.

\section{A4. PBPK model application and comparison with default}

Present the output associated with the range of defaults relevant to the particular assessment. This would facilitate the evaluation of the value of PBPK modelling as well as place it in the context of relative ability to reduce PK uncertainty in a specific risk assessment.

Assess the ability of the PBPK model, relative to the other available approaches, to address the PK uncertainty in the risk assessment based on considerations of the 1) choice of dose metrics, 2) conceptual model and 3) input parameters (Figure A4).

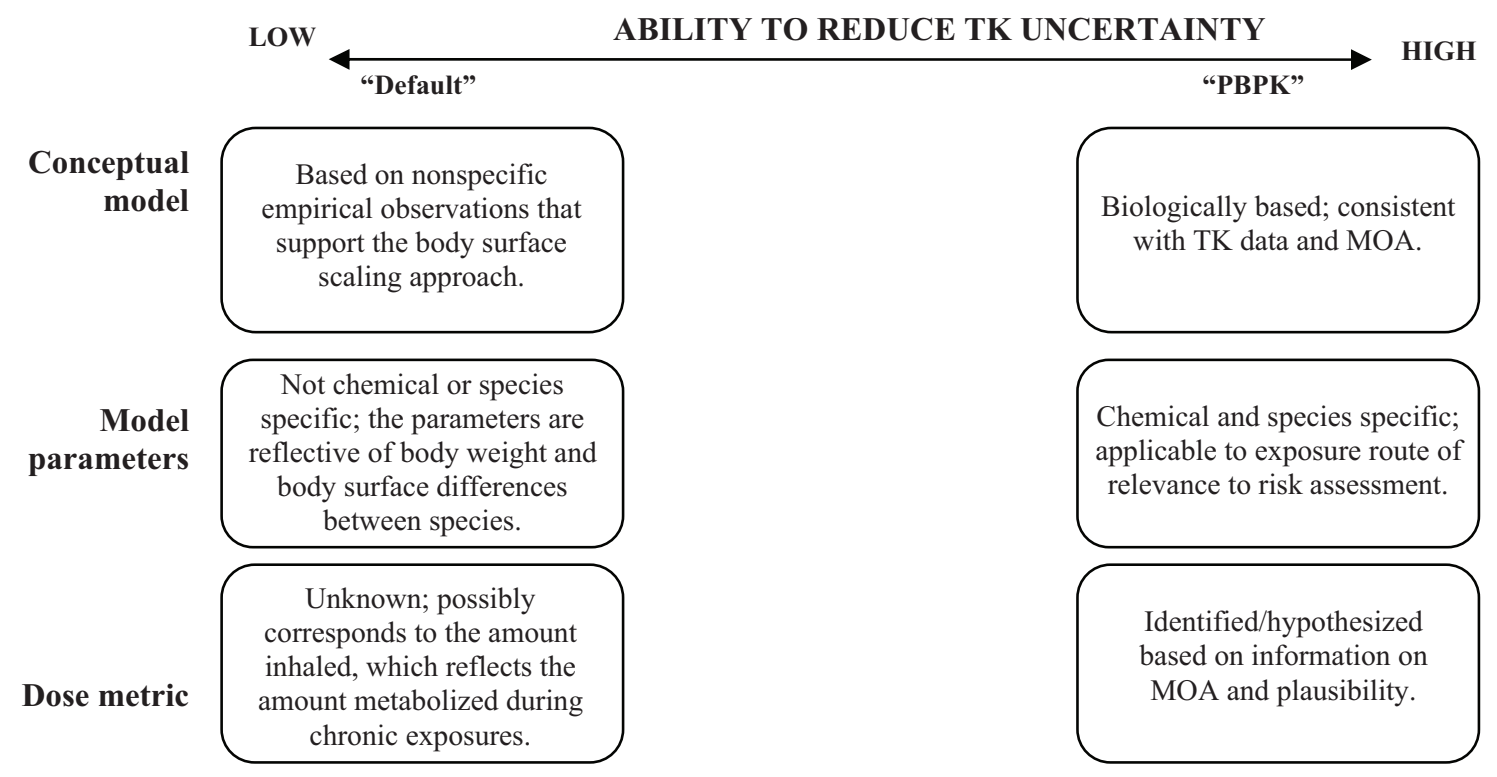

Figure A4: Relative ability of increasingly data-informed approaches in reducing the PK uncertainty associated with interspecies extrapolation for a chemical.

\section{B. CASE-STUDY EXAMPLE: CHEMICAL VC}

\section{B1. Background}

$\mathrm{VC}$ is a volatile chemical for which the risk assessment focuses on deriving a guidance value for the inhalation route based on a cancer bioassay conducted in rats. A central element of this assessment is the rat to human (i.e. interspecies) extrapolation of the inhalation unit risk. This risk assessment application focuses on calculating the human-equivalent exposure concentration of $\mathrm{VC}$ that yields the same level of dose metric as in the responding test species. This case-study will identify a dose metric in the target tissue that relates to tumour response more closely than the external exposure concentration. The resulting measures will identify the external human exposure that results in the same level of the dose metric as in the responding test animal species, using a PBPK model. 


\section{B1.1 Critical effect}

The rich toxicological database on VC indicates that this chemical is a multispecies, multisite carcinogen. VC exposures have been reported to induce benign and malignant tumours in several organs of various species, in both males and females. The most prominent of these is a rare tumour of the liver, namely, angiosarcoma.

In the liver, VC-induced morphological and biochemical effects were apparent in both hepatocytes (origin of the hepatocellular carcinoma) and sinusoidal cells (origin of the angiosarcoma). Hepatic sinusoidal cells appear to be especially susceptible.

In the critical cancer bioassay, female rats were exposed to $\mathrm{VC}$ by inhalation for several weeks and observed throughout their lifetime. Tumour incidence (including liver angiosarcomas) and latency were concentration dependent. Additional studies indicate the occurrence of p53 gene mutations in angiosarcoma tumours induced by VC in SpragueDawley rats as well as in workers with this tumour.

\section{B1.2 Pharmacokinetics}

$\mathrm{VC}$ is absorbed and distributed rapidly following inhalation exposures. Its storage in tissues is limited by its rapid metabolism and excretion. Being lipophilic, VC accumulates to a greater extent in adipose tissues than in blood or other tissues. VC is primarily metabolized to a haloepoxide metabolite, VC-M, via two saturable pathways, one representing low capacityhigh affinity oxidation by CYP2E1 and the other representing higher capacity-lower affinity oxidation by other CYP isozymes (Figure B1). VC-M, which is reactive and short-lived, is scavenged effectively by GSH. In vivo studies in rats and mice indicate that the tissue GSH levels can be depleted following VC exposure due to conjugation with VC-M and that the extent of GSH conjugation is proportional to exposure concentrations up to about $250 \mathrm{mg} / \mathrm{m}^{3}$. At higher exposure concentrations, several experiments indicated saturation of GSH and oxidative metabolism. Saturation of the metabolism of VC also occurs in other species but was not reached in humans exposed to concentrations up to about $60 \mathrm{mg} / \mathrm{m}^{3}$.

\section{B1.3 Mode of action/relevant dose metric}

Key events in the hypothesized mode of induction of tumours by VC are schematically presented in Figure B2. The substantial supporting database, for which the weight of evidence is considerable, satisfies criteria of dose-response as well as temporal concordance of key events, strength, consistency, specificity and biological plausibility. The supporting information on MOA and relevant dose metric is described in more detail in the following paragraphs.

Overall, the available evidence suggests a key role for metabolism in the toxicity and carcinogenicity of $\mathrm{VC}$ in both rats and humans. This derives from in vitro evidence in cells, from experimental animals treated to modify GSH and CYP as well as from in vivo studies in which animals were treated to modify GSH content and in which CYP2E1 content was genetically altered. Dose-response data also lend support: the dose-response for tumours indicated a plateau of effect with increasing dose; this is consistent with the hypothesis that metabolic saturation occurs and with what would be expected for CYP enzymes. 


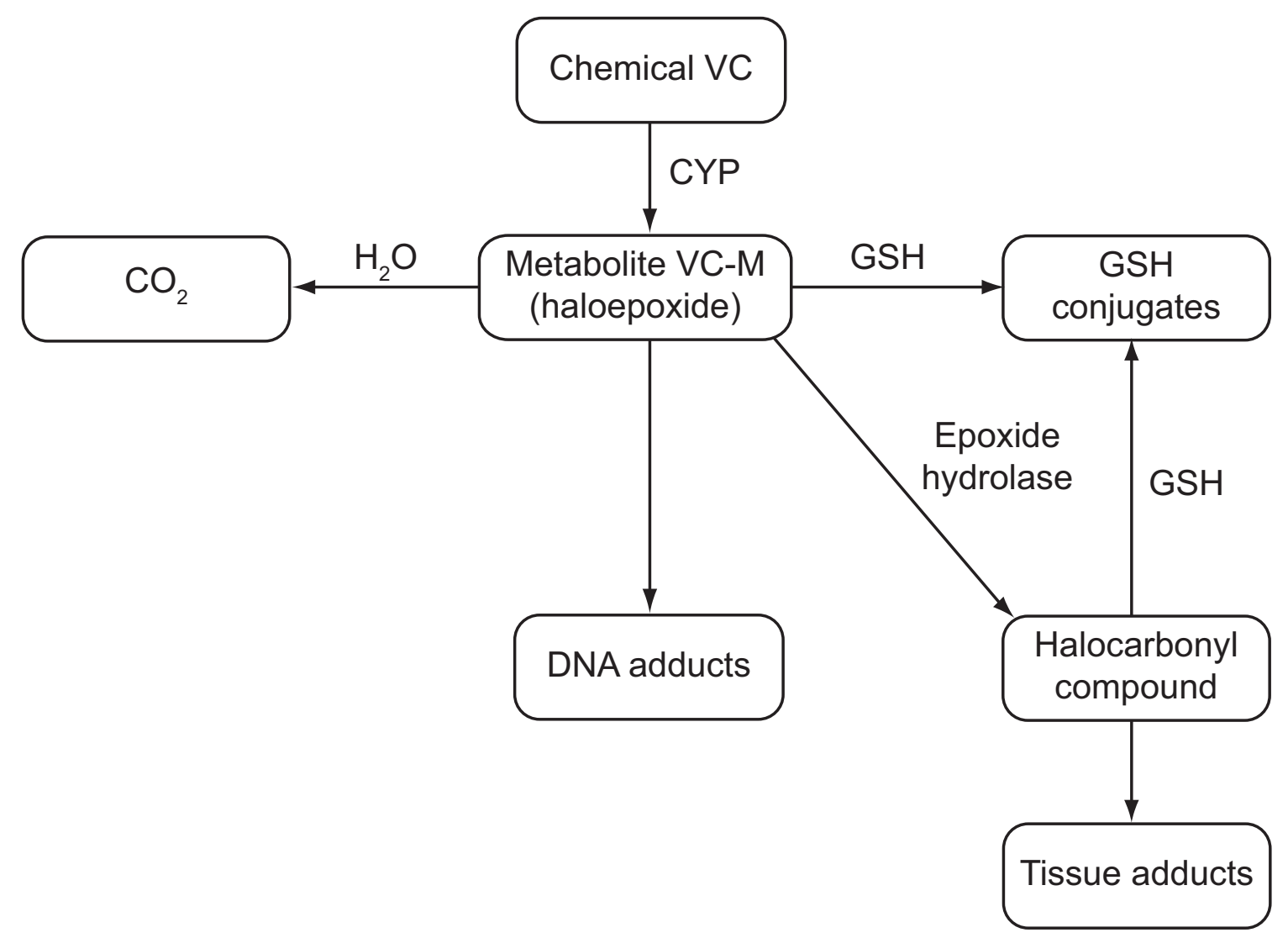

Figure B1: Schematic of the metabolic fate of chemical VC in rats and humans.

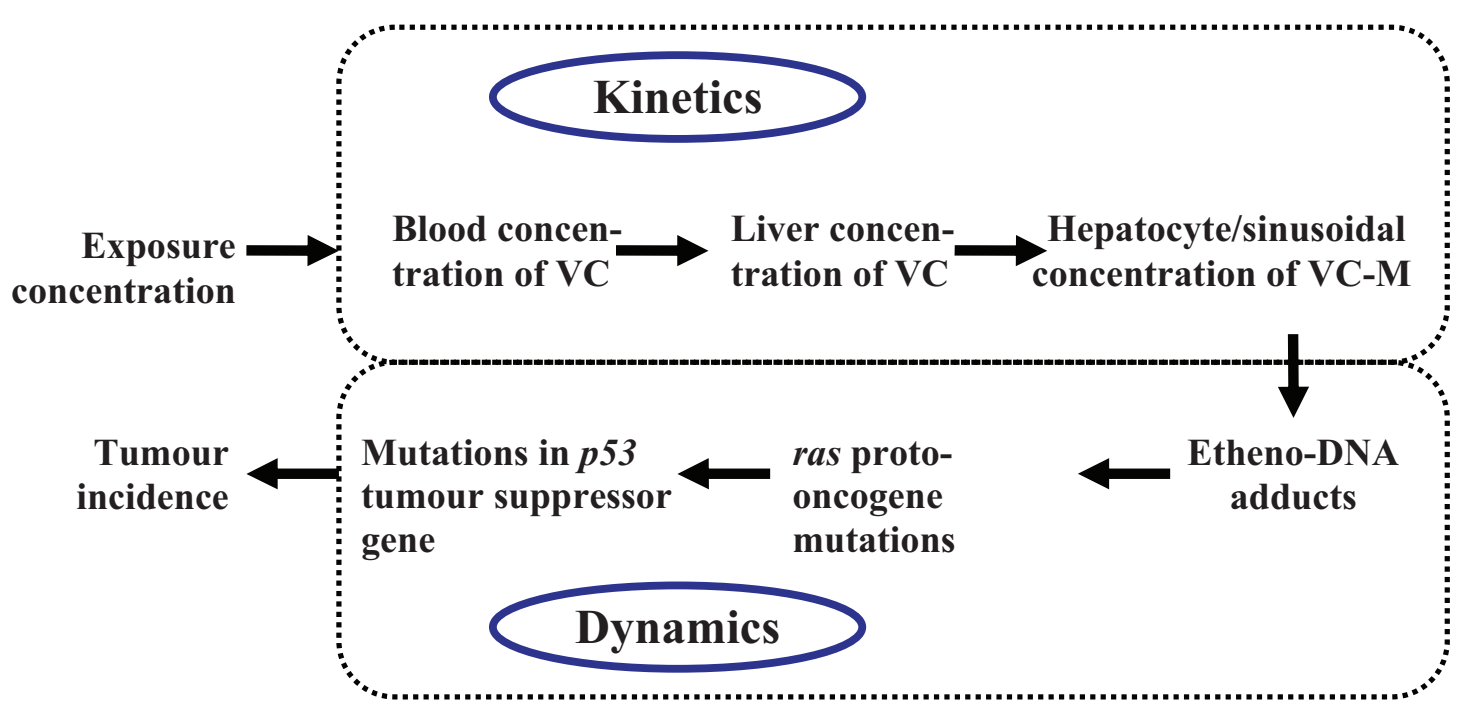

Figure B2: Schematic representation of some key elements of the MOA for the critical doseresponse relationship for chemical VC.

The role of CYP2E1 is further supported by the results of toxicity studies in knockout mice, in which similar toxicity was observed in knockout and wild types at high concentrations (which recruit CYP forms other than CYP2E1 to the reaction). The role of CYP2E1 is additionally supported based on the marked resistance to hepatotoxicity in knockout versus wild-type mice at low concentrations. Together, these data sets indicate that a CYP2E1- 
derived metabolite is toxicologically active and that GSH plays an important role in scavenging this active metabolite.

Mutagenic effects have also been reported in a human cell line containing cloned CYP2E1, capable of metabolizing VC to VC-M. Mutations in the $p 53$ tumour suppressor gene and the ras proto-oncogene in $\mathrm{VC}$-induced liver tumours from rats and humans have been reported.

In additional support of the role of $\mathrm{VC}-\mathrm{M}$ as the relevant toxic metabolite, the available body of data indicates that $\mathrm{VC}$ itself is not DNA reactive and does not induce mutagenic effects in the absence of metabolic activation systems.

From this compilation of evidence, we can summarize the level of confidence in the choice of dose metrics for the MOA of VC as shown in Table B1.

Table B1: Confidence in the use of plausible dose metrics for chemical VC based on an understanding of MOA for the critical effect ( $N$, none; $L$, low; M, medium; H, high).

\begin{tabular}{lc}
\hline Dose metric options & Confidence based on MOA \\
\hline Exposure concentration of parent chemical & $\mathrm{N}$ \\
Blood concentration of parent chemical & $\mathrm{N}$ \\
Absorbed dose of parent chemical & $\mathrm{L}$ \\
Rate of metabolite production & $\mathrm{M}$ \\
Reactive metabolite concentration in target organ & $\mathrm{H}$ \\
Mutagenic DNA adduct level in target organ & $\mathrm{H}$ \\
\hline
\end{tabular}

It would appear that circulating (systemic) concentrations of VC-M cannot be used as a reliable measure of target tissue exposure, given its reactivity and relatively low stability. The primary target cells for VC carcinogenicity (i.e. hepatic sinusoidal cells), however, only have a very limited capacity to metabolize VC. VC-M formed in the hepatocytes would then appear to be the primary source of its levels in the adjacent sinusoidal cells where angiosarcomas originate. Quantum chemical calculations suggest that the VC-M formed in hepatocytes would be stable enough to reach and react with the DNA in adjacent sinusoidal cells.

\section{B1.4 Scope for PBPK model application}

Information on late key events in the hypothesized MOA is likely to provide the most accurate basis for characterization of interspecies differences in effect. A comparison of estimated levels of the putatively toxic metabolite in the target organ (i.e. levels of VC-M in the liver) still has potential to reduce uncertainty in interspecies extrapolations. Reliable measurements of the concentrations of VC-M (which is short-lived and reactive) in VCexposed rats or humans cannot be obtained. The levels of VC-M in rats and humans can be predicted using a PBPK model that takes into account much more chemical-specific information (i.e. physicochemical and biochemical constants) and species-related physiology (weights of organs and tissues and blood flows) than do default approaches, which consider much less information on species differences (e.g. body surface scaling).

In the case of chemical VC, then, the PBPK model application would address the following question: 
What is the human-equivalent concentration of a risk-specific inhalation exposure in rodents, on the basis of equivalent dose metric at the target tissue?

In addition to answering this specific risk assessment question, the PBPK modelling approach might also facilitate the comparison of cancer estimates from epidemiological studies with those derived from test animal bioassays, on the basis of a common dose metric of relevance to the MOA of VC. It is important to recognize in this context that interspecies comparisons are based on measures of central tendency of PK determinants of toxicity.

\section{B2. PBPK model: characterization and evaluation}

\section{B2.1 Model capability and selection}

Given that the cancer risk assessment for $\mathrm{VC}$ focuses on the inhalation route of exposure, the PBPK models selected should be able to simulate potential dose metrics related to VC-M (e.g. total amount metabolized per gram liver, amount metabolized but not conjugated with GSH per gram liver) in both adult rats (test animals) and adult humans exposed by this exposure route.

\section{B2.2 Model structure and biological characterization}

The PBPK model for VC consists of four tissue compartments: liver, adipose tissue, richly perfused tissue compartment, which includes all of the organs except the liver, and poorly perfused tissue compartment, which represents muscles and skin (Figure B3). Absorption is through the pulmonary route, and exhalation of $\mathrm{VC}$ is described as a function of the blood VC concentration, alveolar ventilation rate and blood:air partition coefficient. The distribution of $\mathrm{VC}$ from blood to tissues is described as a perfusion-limited process, determined by tissue blood flow, tissue:blood partition coefficients and tissue volumes.

Metabolism in the liver is characterized by two saturable pathways: 1) high affinity and low capacity (CYP2E1) and 2) low affinity and high capacity (possibly representing one or more CYP2C isozymes). The PBPK model describes the production of the haloepoxide, its subsequent metabolism to carbon dioxide and its reaction with GSH. It also contains a GSH module that allows the simulation of the depletion of GSH following exposure to VC. GSH is an effective scavenger of the reactive metabolite VC-M through conjugation; therefore, as GSH is depleted, a greater quantity of VC-M would interact with the DNA and other macromolecules in the target tissue.

\section{B2.3 Parameter estimation and analysis}

The physiological parameters in test animals were computed on the basis of the body weight information, whereas the human physiological values were obtained from the literature. Species-specific blood:air partition coefficients and rat tissue:air partition coefficients for $\mathrm{VC}$, determined in vitro, were obtained from the literature. The tissue:blood partition coefficients were calculated by dividing the rat tissue:air partition coefficients by the speciesspecific blood:air partition coefficients. The affinity and maximal velocity for metabolism were set based on iterative fitting to in vivo data (total metabolism, GSH depletion). 


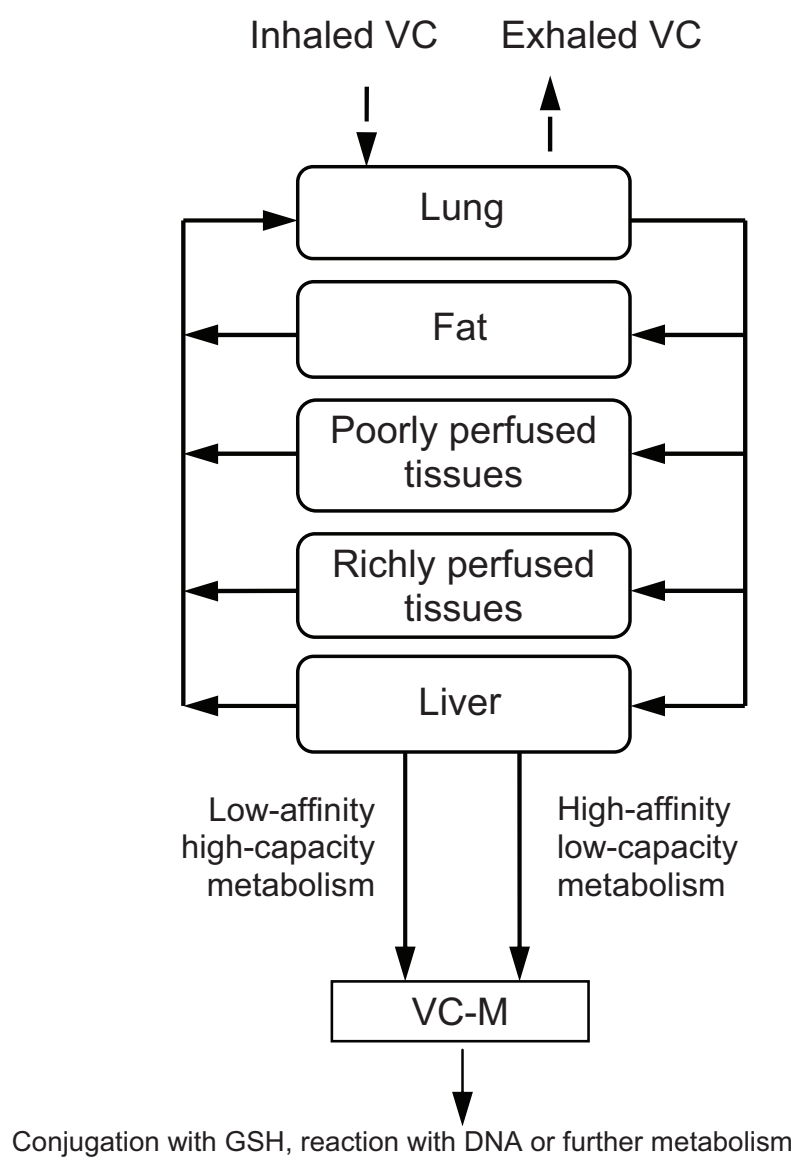

Figure B3: Structure of the PBPK model for chemical VC in rats and humans.

Sensitivity analysis indicated that the body weight, alveolar ventilation rate, cardiac output, blood flow to liver, volume of liver, blood:air partition coefficient as well as the maximal velocity and affinity for the first saturable pathway are the key model parameters in both rats and humans with regard to the dose metric predictions of relevance to $\mathrm{VC}$ risk assessment (see sections B2.4 and B4).

The values and sources of the key parameters of the VC PBPK model are listed in Table B1.1 in Appendix B1.

\section{B2.4 Purpose-specific model evaluation}

The reliability and relevance of the PBPK model for VC were evaluated for the specific purpose for which it is intended - that is, to conduct interspecies extrapolation of the PK of VC. Model evaluation is focused on establishing confidence in the model structure and parameters as well as its ability to adequately predict the behaviour, particularly the dose metrics, to facilitate the rat to human extrapolation of the exposure concentration of VC.

Table B2 lists the experimental data in rats and humans that were simulated by the PBPK model. Visual comparison of simulations and data indicates that model estimations simulate the shape of the time course PK data in rats and humans. The results show that the model consistently reproduces the general trend of the data (i.e. peaks, bumps and valleys, saturation of metabolism) rather than just portions of one or more data sets. Further, the model simulations are close (i.e. within a factor of 2 on average) to the experimental data, which in 
reality constitute only one sample of the hypothetically plausible range of values. As the test animals (or humans) used in estimating parameters versus those used in generating the various PK data for model evaluation purposes are not the same, some level of discordance is expected. In the case of $\mathrm{VC}$, the PBPK model simulations were within a factor of 2 of the experimental data on respiratory uptake, the amount metabolized, change in hepatic GSH concentrations (indicative of VC-M formed in liver) as well as the PK profile following inhalation exposure. The model simulations of the amount metabolized, which is directly reflective of the amount of VC-M produced, are supported by the simulations of data on depletion of GSH, which is representative of the amount of VC-M scavenged following exposure to $\mathrm{VC}$.

Table B2: List of PK studies used for evaluating the PBPK model for VC.

\begin{tabular}{|c|c|c|c|}
\hline Species & Dose/exposure concentration & Route & Data \\
\hline Rat & $\begin{array}{l}4 \mathrm{~h} \text { exposure to } 15,50,150 \text {, } \\
1500 \text { and } 15000 \mathrm{mg} / \mathrm{m}^{3}\end{array}$ & Inhalation & $\begin{array}{l}\text { GSH concentration in liver at } 0,20 \text { and } \\
44 \mathrm{~h} \text { following } 4 \mathrm{~h} \text { exposure }\end{array}$ \\
\hline Rat & $\begin{array}{l}6 \mathrm{~h} \text { exposure to } 3-12800 \\
\mathrm{mg} / \mathrm{m}^{3}\end{array}$ & Inhalation & $\begin{array}{l}\text { GSH concentration in liver immediately } \\
\text { following } 6 \text { h exposure }\end{array}$ \\
\hline Rat & $\begin{array}{l}6 \mathrm{~h} \text { exposure to } 3-12800 \\
\mathrm{mg} / \mathrm{m}^{3}\end{array}$ & Inhalation & $\begin{array}{l}\text { Total amount metabolized during } 6 \mathrm{~h} \\
\text { inhalation exposures }\end{array}$ \\
\hline Rat & $\begin{array}{l}\text { Closed chamber concentrations } \\
\text { of } 610,1100,1670,2110,2940 \\
\text { or } 3580 \mathrm{mg} / \mathrm{m}^{3}\end{array}$ & Inhalation & $\begin{array}{l}\text { Chamber concentrations reflective of } \\
\text { the respiratory uptake of VC by the } \\
\text { organism }\end{array}$ \\
\hline Rat & 0.05-100 mg/kg body weight & $\begin{array}{l}\text { Oral (in } \\
\text { corn oil) }\end{array}$ & $\begin{array}{l}\text { Total amount expired as } \mathrm{CO}_{2} \text { (as \% } \\
\text { total metabolism) }\end{array}$ \\
\hline Human & $\begin{array}{l}\text { Closed chamber exposure to } \\
25.6 \mathrm{mg} / \mathrm{m}^{3}\end{array}$ & Inhalation & $\begin{array}{l}\text { Chamber concentrations reflective of } \\
\text { the uptake by volunteers during } 6 \mathrm{~h} \\
\text { exposures }\end{array}$ \\
\hline Human & $\begin{array}{l}15-30 \mathrm{~min} \text { exposures to } 6.4 \\
\mathrm{mg} / \mathrm{m}^{3}\end{array}$ & Inhalation & Exhaled concentration of VC \\
\hline
\end{tabular}

Degree of similarity of model simulations to experimental data is not the sole manner of determining the level of confidence associated with a PBPK model. Rather, the consideration of the following aspects should be an integral part of this process (see section 3.7):

- Biological basis: Do the model structure and parameters have a reasonable biological basis?

- Model simulations of data: How well does the PBPK model reproduce the chemicalspecific TK data under various experimental or exposure conditions?

- Reliability (testing of model, uncertainty and sensitivity): How reliable is the PBPK model with regard to its predictions of dose metrics relevant to risk assessment?

Based on consideration of the biological basis, model performance and reliability of dose metric predictions, there is a high level of confidence in the VC PBPK model for the conduct of interspecies (rat to human) extrapolation using dose metrics related to VC-M (Table B3).

The results of the uncertainty/sensitivity analyses for the rat PBPK model for chemical VC $\left(128 \mathrm{mg} / \mathrm{m}^{3}, 4 \mathrm{~h} /\right.$ day, inhalation) are shown in Figure B4. 
Table B3: Purpose-specific evaluation of the PBPK model for chemical VC.

\section{Model evaluation aspect}

Biological basis

Model simulations of data

Reliability (model testing, uncertainty and sensitivity)

\section{Observation}

The model parameters, structure and assumptions have reasonable biological basis.

Model consistently simulates the time course data (i.e. bumps and valleys) relating to the respiratory uptake, amount metabolized as well as hepatic GSH concentrations following inhalation exposures. The PBPK model, with a single set of input parameters, is capable of reproducing (qualitatively: bumps and valleys; quantitatively: within a factor of 2) the TK data for various inhalation exposure concentrations in rats and humans.

Experimental data on dose metric (i.e. VC-M in liver) are not available for comparison with model. However, model simulations of total amount metabolized and GSH depletion (which are indicative of VC-M produced in liver) were within a factor of 2 of the experimental values for various exposure doses and scenarios in the rat. A sensitivity analysis of the parameters of the PBPK model showed that there was no amplification of error from inputs to outputs (see below for summary of results). An uncertainty/variability analysis (4 realizations, 500 simulations/realization) was conducted to evaluate the impact of parameter uncertainty and variability on risk prediction. The 95th percentile of the distribution of the upper confidence limit risk was approximately within a factor of 2 of the mean upper confidence limit risk, indicating the reliability of the model structure and parameters.

\begin{tabular}{|c|c|c|c|c|}
\hline & & \multicolumn{3}{|c|}{ UNCERTAINTY } \\
\hline & & High & Medium & Low \\
\hline \multirow{3}{*}{ 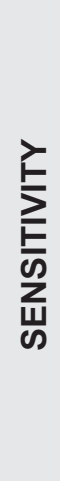 } & 号 & & Cardiac output & $\begin{array}{c}\text { Liver volume } \\
\text { Blood:air partition coefficient } \\
\text { Liver blood flow }\end{array}$ \\
\hline & $\begin{array}{l}\frac{\mathrm{g}}{\bar{D}} \\
\frac{\overline{7}}{0} \\
\frac{0}{2}\end{array}$ & & Pulmonary ventilation & Body weight \\
\hline & 30 & & & $\begin{array}{c}V_{\max } \\
K_{\mathrm{m}}\end{array}$ \\
\hline
\end{tabular}

Figure B4: The output of the uncertainty and sensitivity analyses for the rat VC PBPK model. Sensitivity and uncertainty analysis results are presented as high, medium or low. The values were subjectively scaled based on their absolute impact on the simulated dose metrics (see also Figures 8 and 9).

The summary results for such analyses with the human PBPK model (exposure conditions: $2.6 \mathrm{mg} / \mathrm{m}^{3}$, continuous inhalation exposure) are presented in Figure B5. 


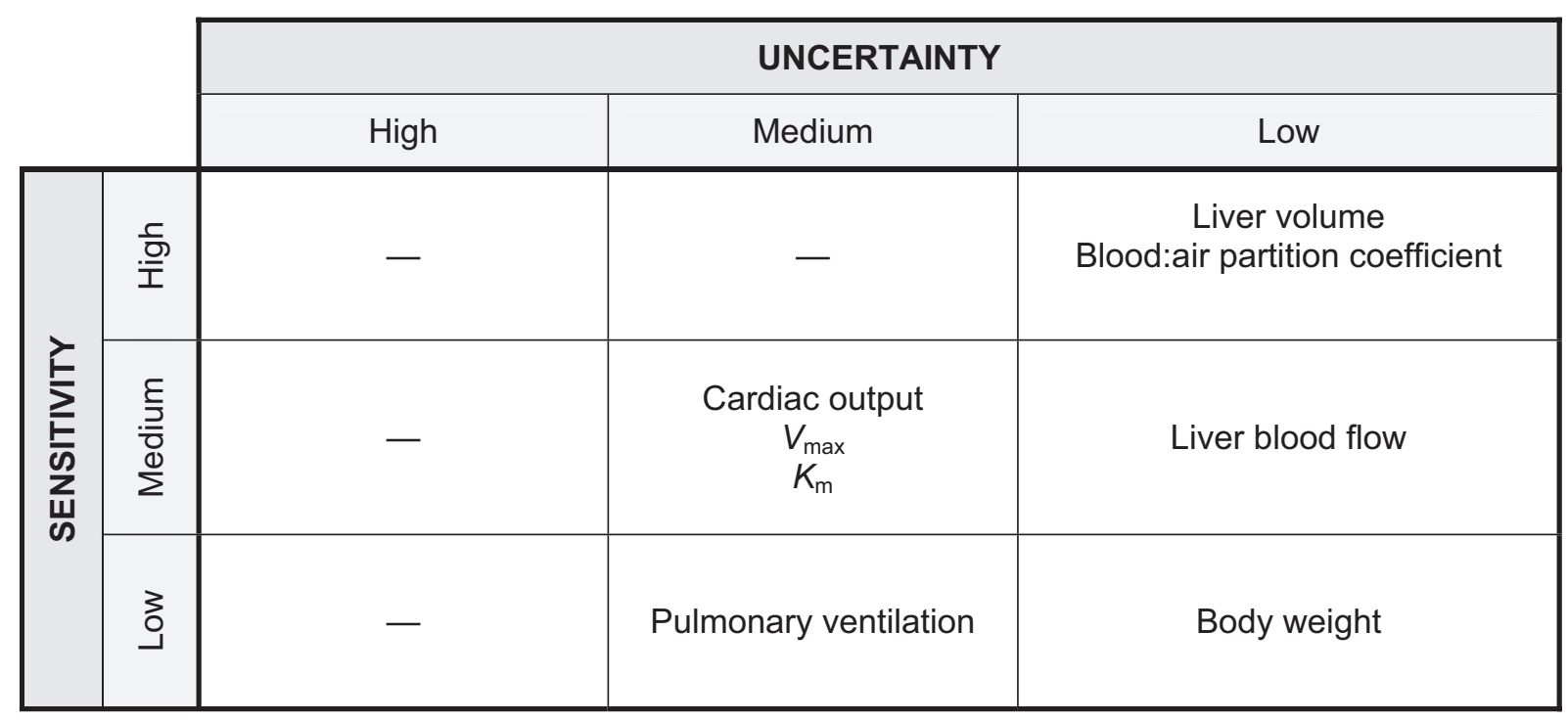

Figure B5: The output of the uncertainty and sensitivity analyses for the human VC PBPK model. Sensitivity and uncertainty analysis results are presented as high, medium or low. Values are scaled in the same manner as in Figure B4.

The results of the sensitivity analysis indicate that the key model parameters are body weight, alveolar ventilation rate, cardiac output, blood flow to liver, volume of liver, blood:air partition coefficient as well as the maximal velocity and affinity for the first saturable pathway. The reliability of the model predictions of dose metrics of relevance to the risk assessment is influenced by the level of uncertainty in the sensitive parameters of the model. Thus, if the highly sensitive parameters are also the ones that are highly uncertain, then the reliability of the model for risk assessment applications would be questionable. In the case of $\mathrm{VC}$, the highly sensitive model parameters exhibit high certainty (or low uncertainty), contributing to a high level of confidence in the use of this model for predicting dose metrics for risk assessment.

\section{B2.5 Model documentation}

The model for VC was implemented in ACSL; model code is available from the corresponding author. Study-specific body weight and other parameters (fat volume and metabolic parameters) are provided along with distributions of parameters used for variability/uncertainty analysis.

\section{B2.6 Model peer review}

The rat and human PBPK models for VC were published in the peer-reviewed literature. Further, independent review of these models and codes was undertaken on two different occasions by government agencies in North America in the context of specific risk assessment applications.

\section{B3. Evaluation of dose metrics}

Empirical evidence from in vitro and in vivo studies suggests a key role for metabolism in the toxicity and carcinogenicity of VC. The dose-response data for tumours indicate the attainment of a plateau for tumour incidence with increasing dose, consistent with VC-M being the toxic moiety formed via a saturable pathway. The role of CYP2E1 is supported by 
the results of toxicity studies in knockout mice, in which similar toxicity was observed in knockout and wild-type mice at high concentrations (which recruit CYP forms other than CYP2E1 to the reaction). These data sets indicate that a CYP2E1-derived VC-M is likely the toxicologically active moiety.

The data on the clearance and lack of reactivity of $\mathrm{VC}$, in conjunction with the observations that:

- mutagenic effects were observed only in systems that were metabolically active (to produce $\mathrm{VC}-\mathrm{M}$ ),

- mutagenic effects were further exacerbated following the induction of liver CYP enzymes and

- base pair mutations, indicative of the role of etheno adducts formed by VC-M, occurred following $\mathrm{VC}$ exposures,

indicate that the tissue dose of $\mathrm{VC}-\mathrm{M}$, rather than that of $\mathrm{VC}$, would be more closely related to the tumour response. In other words, VC-M is more closely related than VC to the tumour response, such that an appropriate measure of "dose to target" should be reflective of the amount of VC-M formed in liver. Circulating concentrations of this metabolite, however, cannot be used as a reliable measure of internal dose, given its reactivity and relatively low stability.

Table B4 provides a comparison of the level of confidence in the use of alternative dose metrics, based on the MOA and the capability of the PBPK model.

Table B4: Confidence in the use of plausible dose metrics for chemical VC based on an understanding of MOA for the critical effect and PBPK modelling results (N, none; L, low; M, medium; H, high; N/A, not available).

\begin{tabular}{lcc}
\hline Dose metric options & $\begin{array}{c}\text { Confidence } \\
\text { based on MOA }\end{array}$ & $\begin{array}{c}\text { Confidence in } \\
\text { simulation based on } \\
\text { PBPK modelling }\end{array}$ \\
\hline Exposure concentration of parent chemical & $\mathrm{N}$ & - \\
Blood concentration of parent chemical & $\mathrm{N}$ & $\mathrm{H}$ \\
Absorbed dose of parent chemical & $\mathrm{L}$ & $\mathrm{H}$ \\
Rate of metabolite production & $\mathrm{M}$ & $\mathrm{H}$ \\
Reactive metabolite concentration in target organ & $\mathrm{H}$ & $\mathrm{L}$ \\
Mutagenic DNA adduct level in target organ & $\mathrm{H}$ & $\mathrm{N} / \mathrm{A}$ \\
\hline
\end{tabular}

Given these considerations, two alternative dose metrics simulated with the PBPK model were evaluated: 1) the daily average amount of $\mathrm{VC}-\mathrm{M}$ generated in the liver divided by the volume of liver (i.e. the tissue into which it is produced) (dose metric-1); and 2) the daily total amount of metabolite not detoxified by reaction with GSH, again divided by the volume of the liver (dose metric-2). The use of dose metric-1 simulated by the PBPK model shows that the concentration of the actual carcinogenic moiety (or the extent of the crucial event associated with the cellular transformation) is linearly related to this pseudo-concentration of reactive intermediate except at high concentrations at which GSH is depleted significantly, as depicted in Figure B6(A). 
A

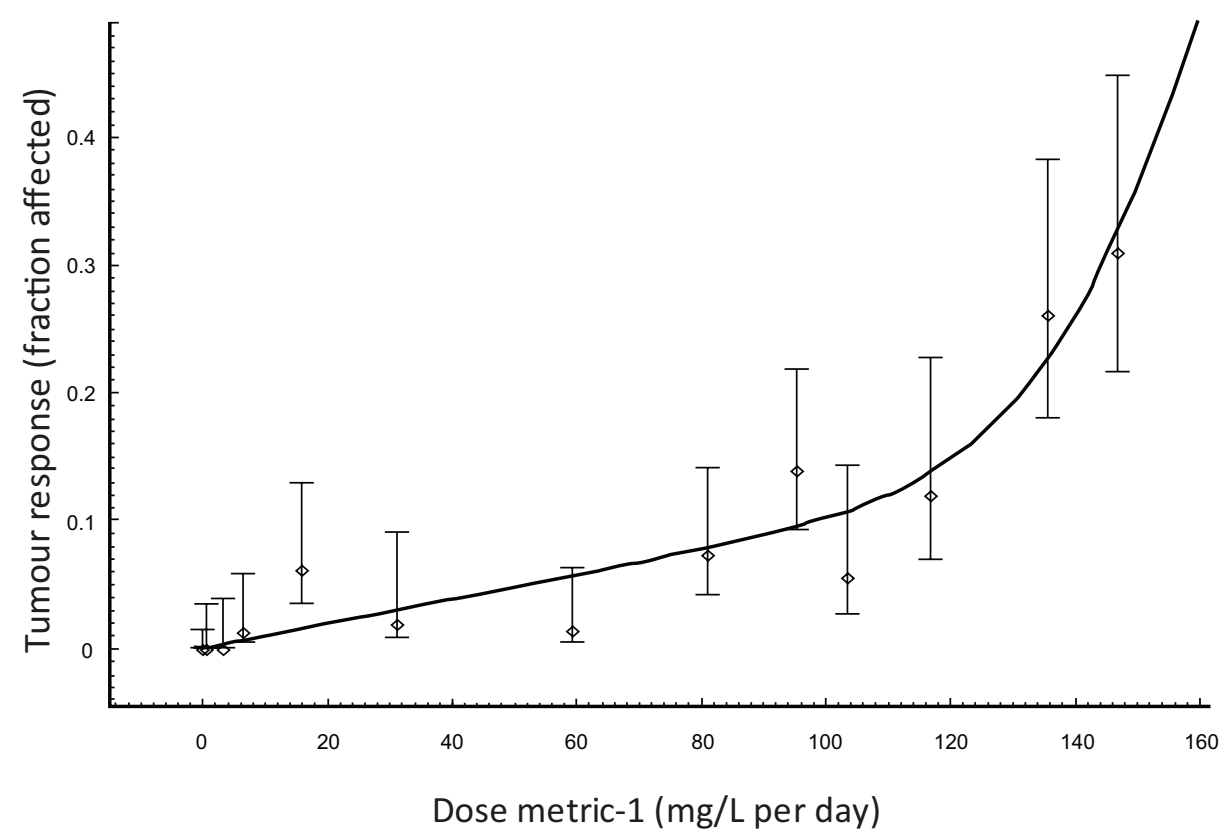

B

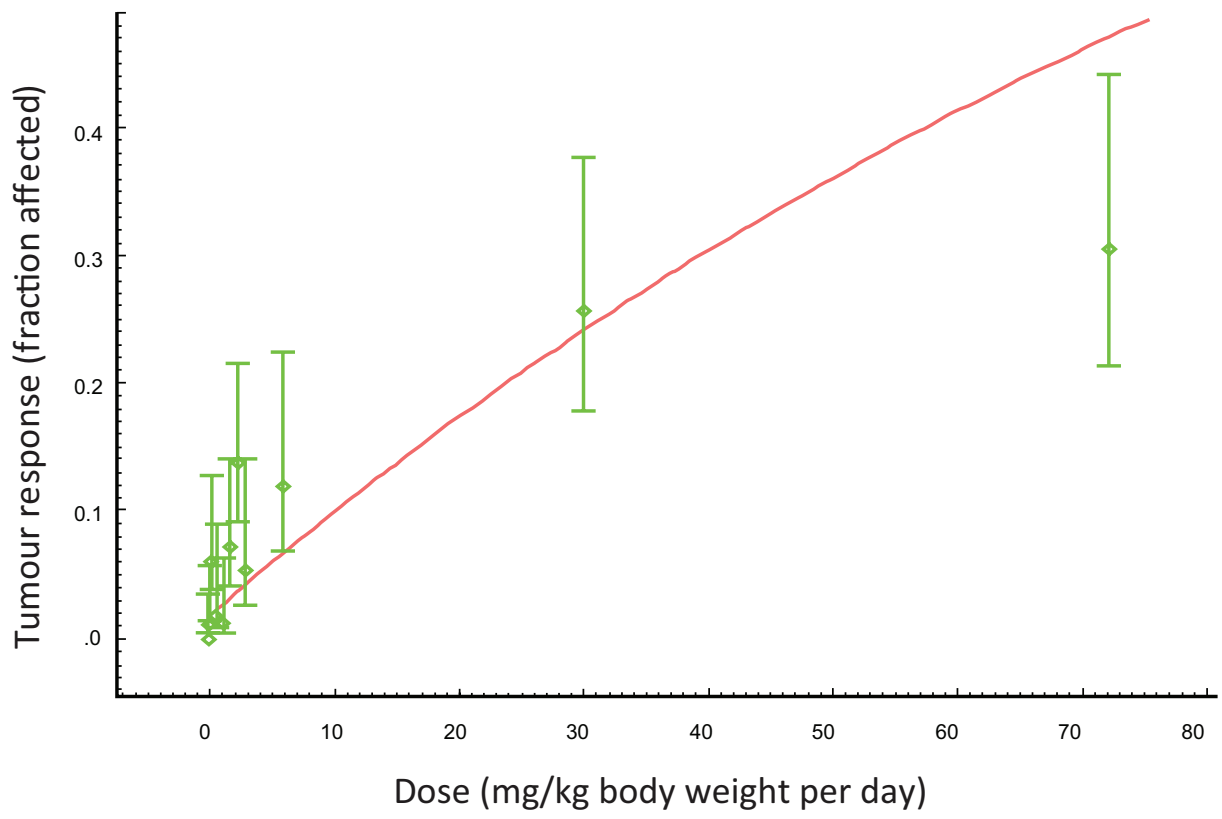

Figure B6: Dose-response modelling for VC-induced liver angiosarcoma using (A) PBPK model simulations of dose metric-1 (milligrams metabolized per litre of liver per day; note that the increased potency at highest concentrations reflects depletion of GSH) and (B) applied dose (milligram per kilogram body weight per day).

In the case of $\mathrm{VC}$, it was not possible to choose one of dose metric-1 and dose metric- 2 on the basis of their description of dose-response data or results of the goodness of fit analysis. Further, the risk estimates were insensitive to the choice of dose metric that is derived from quantitative estimates of the metabolism of VC. Dose metric-1 is a biologically plausible measure of dose relevant to the MOA of $\mathrm{VC}$ and is also associated with greater certainty of prediction in humans, given that it requires fewer parameters to be estimated (i.e. does not require estimates of species-specific parameters for the GSH conjugation module of the 
PBPK model). Further, when the potency of VC liver carcinogenicity was expressed in terms of dose metric-1 (i.e. the daily amount of VC-M generated in the liver divided by the volume of liver) averaged over the lifetime, essentially the same potency was calculated from inhalation and oral studies in the mouse and rat, as well as from occupational inhalation exposures in humans. In this regard, it is useful to note that the lifetime average daily dose (equivalent to average daily AUC), rather than the total lifetime dose (or lifetime total AUC), has been found empirically to provide a better cross-species extrapolation of carcinogenic potency.

\section{B4. PBPK model application and comparison with default}

The dose-response characterization based on external dose (i.e. default approach) and dose metric-1 (i.e. daily average amount of VC-M produced per litre of liver obtained with the PBPK model) are depicted in Figure B6.

The default approach for the kinetic component of interspecies extrapolation for $\mathrm{VC}$ consists of 1) calculating inhaled dose in the rat using an assumed ventilation rate $\left(0.223 \mathrm{~m}^{3} /\right.$ day $)$, body weight $(0.35 \mathrm{~kg})$ and fraction absorbed $(0.5) ; 2)$ adjusting the slope factor as the ratio of human to rat body weights raised to the one-third power: $(60 / 0.35)^{1 / 3}$; and 3$)$ calculating human-equivalent concentration using an assumed ventilation rate $\left(20 \mathrm{~m}^{3} /\right.$ day $)$, body weight $(60 \mathrm{~kg})$ and fraction absorbed (1.0). The net effect of these default assumptions yields a human risk at a given $\mathrm{VC}$ concentration that is greater than that for the rat by a factor of 5.6, as shown below:

$$
\text { Dose }_{\text {rat }} / \text { Dose }_{\text {human }}=(0.5 \times 0.223 / 0.35) \times 5.85 /(1.0 \times 20 / 60)=5.6
$$

These same adjustments, conducted on the basis of dose metric-1 calculated by the PBPK model, indicate that a continuous exposure at $2.6 \mathrm{mg} / \mathrm{m}^{3} \mathrm{VC}$ would yield $12.9 \mathrm{mg}$ metabolized per litre of liver per day in the rat, compared with just $1.74 \mathrm{mg}$ metabolized per litre of liver per day in the human. Taken together, the PBPK approach predicts that the human risk at a given $\mathrm{VC}$ concentration would be less than that for the rat by a factor of 7.4:

$$
\text { Dose metric- } 1_{\text {rat }} / \text { Dose metric- } 1_{\text {human }}=12.9 / 1.74=7.4
$$

In other words, the total difference between the two approaches is: $5.6 \times 7.4=41$. That is, the PBPK-based approach predicts a 41-fold lower human risk than the default approach for the chemical VC. The animal-based estimate of the unit cancer risk from lifetime exposure to VC obtained with PBPK modelling $\left(1.1 \times 10^{-6}\right.$ per $\left.\mu \mathrm{g} / \mathrm{m}^{3}\right)$, however, is consistent with the range of risk estimates from the epidemiological studies $\left(0.2-1.7 \times 10^{-6}\right.$ per $\left.\mu \mathrm{g} / \mathrm{m}^{3}\right)$.

The application of the default approach to adjust for interspecies differences in PK of VC is uncertain, as the dose metric (i.e. amount of VC-M formed per unit volume of target tissue) is not necessarily a simple function of body weight, body surface area and ventilation rate. For the chemical VC, as the tumour response is more closely related to the rate of reactive metabolite produced in the target tissue rather than to the parent chemical concentration in the air or the amount inhaled, the use of VC-M as the dose measure in the risk assessment is believed to be more certain than the default approach for interspecies extrapolation. Indeed, body surface scaling is not applied in some jurisdictions when there is sufficient evidence that toxicity is mediated through a metabolite (i.e. application of body surface area scaling is less justified than when toxicity is mediated through the parent compound). 
Given that the interspecies extrapolation aspect focuses on the evaluation of the central tendency (i.e. the ratio of median values of the kinetic parameters between test animals and humans), deterministic PBPK models are used for this purpose (see section 3.7.2). Regarding the uncertainty in the "average" values of parameters of a PBPK model used for interspecies extrapolation, a relevant question is:

What is the impact of this parameter uncertainty on the simulations of dose metrics relative to the uncertainty associated with the use of the available alternative approach (e.g. the default)?

The uncertainty related to the available alternative (i.e. default) approach arises from the conceptual model (based on nonspecific empirical observations), parameters (e.g. same for all chemicals and species; based on average body weight of $0.35 \mathrm{~kg}$ for the rat and $60 \mathrm{~kg}$ for humans) as well as the toxic moiety (i.e. unknown) (Figure B7). In this regard, PBPK models offer an opportunity to incorporate more data to inform the adequacy of or reduce the uncertainty associated with the default approaches, by simulating dose metric-1 for VC on the basis of relevant physiological (breathing rate, volume of the target tissue, liver blood flow rate), biochemical (rate of metabolic conversion of $\mathrm{VC}$ to $\mathrm{VC}-\mathrm{M}$ ) and physicochemical (partitioning of $\mathrm{VC}$ between blood and air, partitioning of $\mathrm{VC}$ between tissues and blood) determinants in both test species and humans. A sensitivity analysis of the parameters of the PBPK model showed that there was no amplification of error from inputs to outputs. A Monte Carlo uncertainty/variability analysis (4 realizations, 500 simulations/realization) was conducted to evaluate the impact of parameter uncertainty and variability on risk prediction. The 95th percentile of the distribution of the upper confidence limit risk was approximately within a factor of 2 of the mean upper confidence limit risk, indicating the robustness and reliability of the PBPK model for VC.

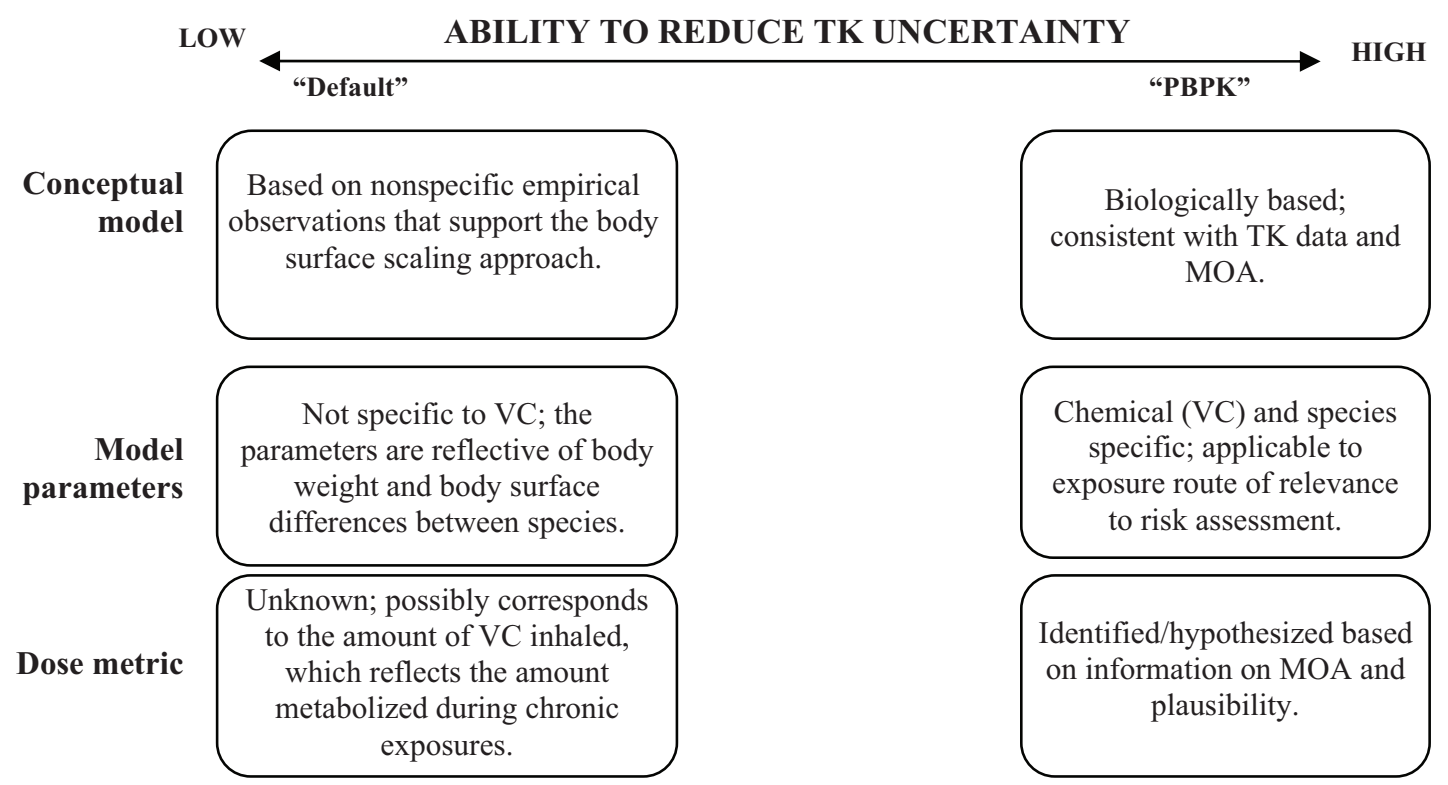

Figure B7: Relative ability of increasingly data-informed approaches in reducing the TK uncertainty associated with interspecies extrapolation for VC. 


\section{APPENDIX B1}

Table B1.1: Critical PBPK model parameters for chemical VC. ${ }^{a}$

\begin{tabular}{|c|c|c|c|}
\hline Parameter & Rat $^{a}$ & Human $^{a}$ & Source \\
\hline Body weight $(\mathrm{kg})$ & $(11)^{b}$ & $70.0(30)$ & Literature or study-specific values \\
\hline $\begin{array}{l}\text { Alveolar ventilation }(\mathrm{l} / \mathrm{h} \text {, } \\
1 \mathrm{~kg} \text { animal) }\end{array}$ & $21.0(58)$ & $24.0(16)$ & Scaled to body weight \\
\hline $\begin{array}{l}\text { Cardiac output }(\mathrm{l} / \mathrm{h}, \\
1 \mathrm{~kg} \text { animal) }\end{array}$ & $18.0(9)$ & $16.5(9)$ & Scaled to body weight \\
\hline $\begin{array}{l}\text { Flow to liver (as fraction of } \\
\text { cardiac output) }\end{array}$ & $0.25(96)$ & $0.26(35)$ & Literature \\
\hline $\begin{array}{l}\text { Volume of liver (as fraction } \\
\text { of body weight) }\end{array}$ & $0.04(6)$ & $0.026(5)$ & Literature \\
\hline $\begin{array}{l}\text { Blood:air partition } \\
\text { coefficient }\end{array}$ & $2.4(15)$ & $1.16(10)$ & $\begin{array}{l}\text { In vitro (vial equilibration) data obtained } \\
\text { from literature }\end{array}$ \\
\hline $\begin{array}{l}\text { Maximum velocity of first } \\
\text { saturable pathway (mg/h, } \\
1 \mathrm{~kg} \text { animal) }\end{array}$ & $4.0(20)$ & $4.0(30)$ & $\begin{array}{l}\text { Fitting to in vivo data from closed } \\
\text { chamber exposures (rats); estimated } \\
\text { from in vivo exposures in non-human } \\
\text { primates (human); } V_{\max } \text { scaled to human } \\
\text { as body weight raised to the } 3 / 4 \text { power }\end{array}$ \\
\hline $\begin{array}{l}\text { Affinity of first saturable } \\
\text { pathway }(\mathrm{mg} / \mathrm{l})\end{array}$ & $0.1(30)$ & $1.0(50)$ & $\begin{array}{l}\text { Literature on competitive interactions } \\
\text { among CYP2E1 substrates (rat); fitting to } \\
\text { in vivo data from closed chamber } \\
\text { exposures (human) }\end{array}$ \\
\hline
\end{tabular}

a Coefficient of variation (CV, in \%) given in parentheses. CV $=100 \times$ standard deviation/mean.

b The value of this parameter was strain and study specific. 


\section{THE HARMONIZATION PROJECT DOCUMENT SERIES}

IPCS risk assessment terminology (No. 1, 2004)

Chemical-specific adjustment factors for interspecies differences and human variability:

Guidance document for use of data in dose/concentration-response assessment (No. 2, 2005)

Principles of characterizing and applying human exposure models (No. 3, 2005)

Part 1: IPCS framework for analysing the relevance of a cancer mode of action for humans and case-studies; Part 2: IPCS framework for analysing the relevance of a non-cancer mode of action for humans (No. 4, 2007)

Skin sensitization in chemical risk assessment (No. 5, 2008)

Uncertainty and data quality in exposure assessment. Part 1: Guidance document on characterizing and communicating uncertainty in exposure assessment; Part 2: Hallmarks of data quality in chemical exposure assessment (No. 6, 2008)

Assessment of combined exposures to multiple chemicals: report of a WHO/IPCS international workshop on aggregate/cumulative risk assessment (No. 7, 2009)

WHO human health risk assessment toolkit: chemical hazards (No. 8, 2010)

Characterization and application of physiologically based pharmacokinetic models in risk assessment (No. 9, 2010) 